
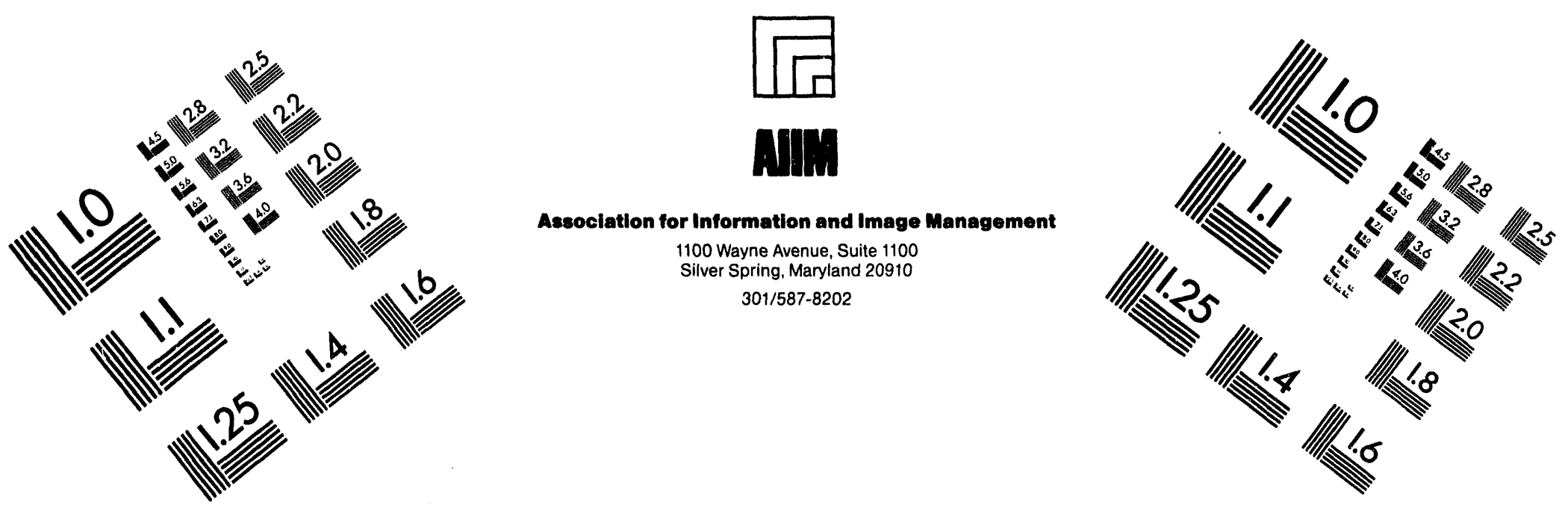

Centimeter

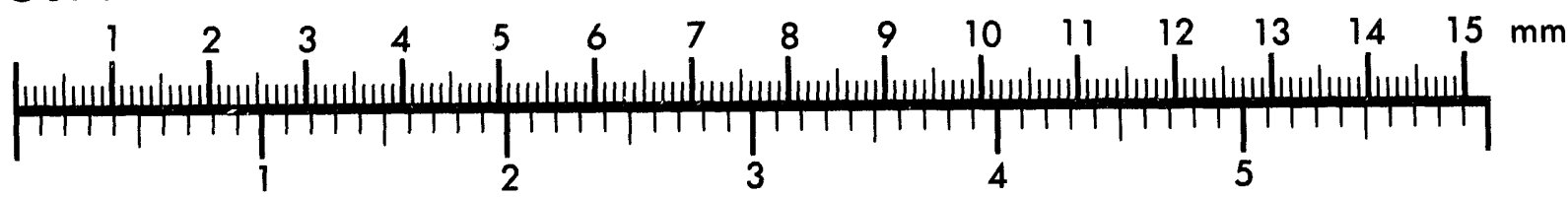
Inches
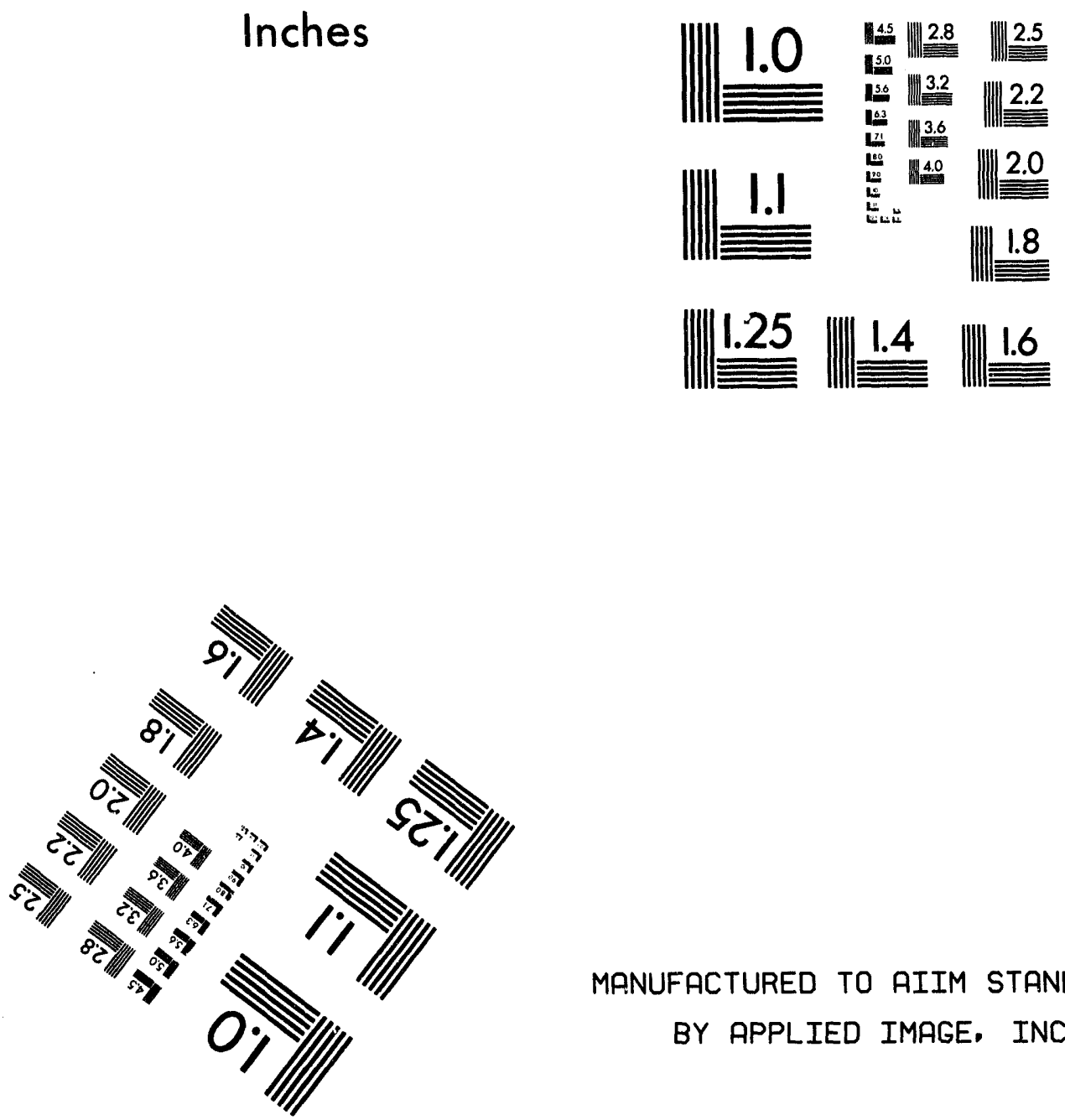

MANUFACTURED TO AIIM STANDARDS BY APPLIED IMAGE, INC.

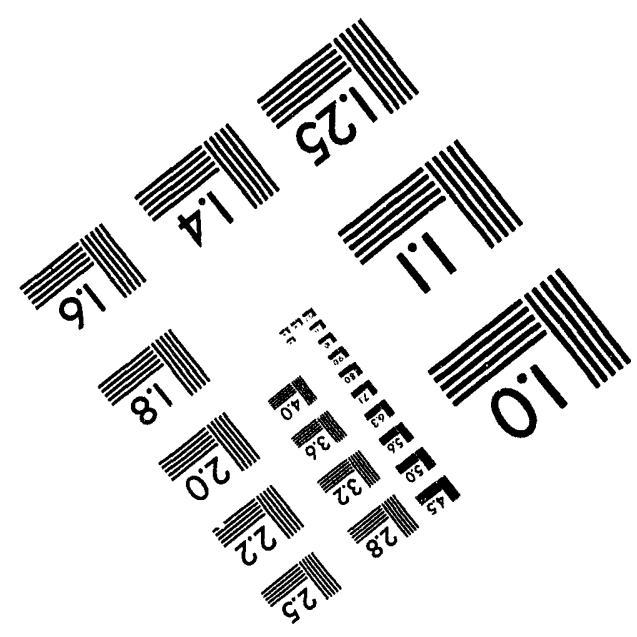



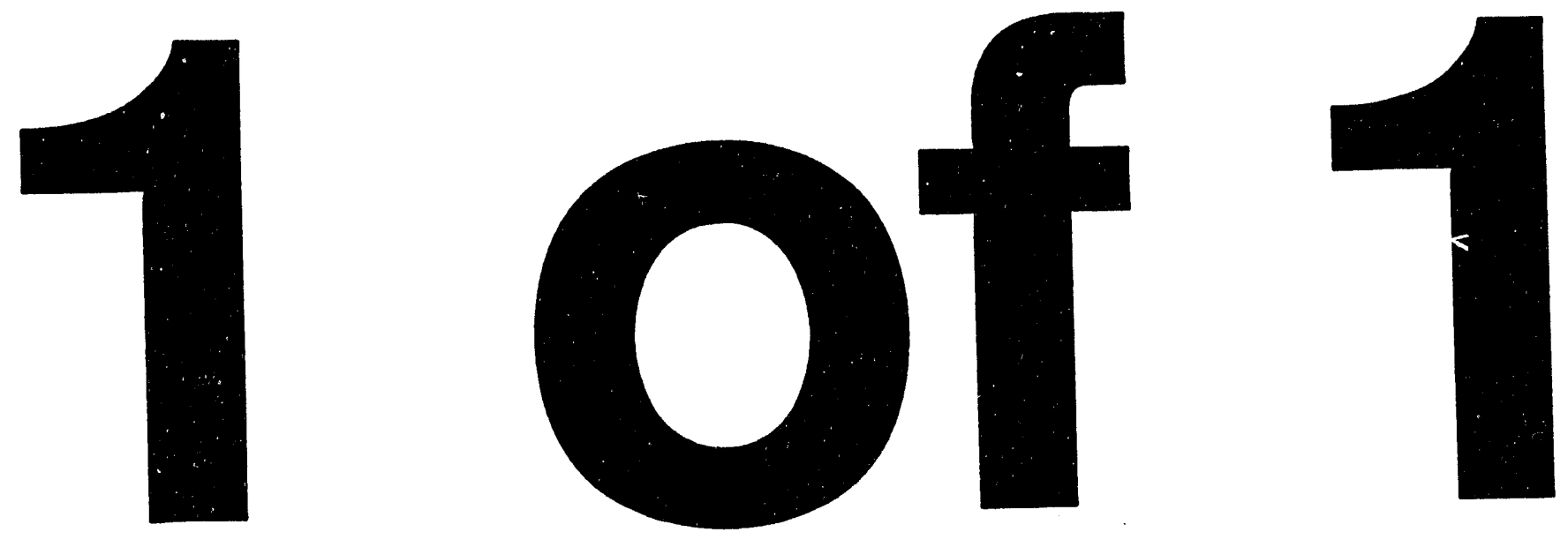
Computing Applications Division

\section{THE LAW LIBRARY - A MULTIGROUP CROSS-SECTION LIBRARY FOR USE IN RADIOACTIVE WASTE ANALYSIS CALCULATIONS}

N. M. Greene, J. W. Arwood, R. Q. Wright, C. V. Parks

Date Published: August 1994

Prepared for the

U.S. Department of Energy

Office of Environment, Safety, and Health

Division of Transportation and Packaging Safety

Budget Activity No. HA 010900000

and

Oak Ridge Y-12 Plant

Packaging Systems Management

Defense Programs and Engineering Services

Prepared by the

OAK RIDGE NATIONAL LABORATORY

Oak Ridge, Tennessee

managed by

MARTIN MARIETTA ENERGY SYSTEMS, INC.,

for the

U.S. DEPARTMENT OF ENERGY

under contract DE-AC05-84OR21400 


\section{CONIENIS}

ACKNOWLEDGMENTS $\ldots \ldots \ldots \ldots \ldots \ldots \ldots \ldots \ldots \ldots \ldots \ldots \ldots \ldots \ldots \ldots$

ABSTRACT $\ldots \ldots \ldots \ldots \ldots \ldots \ldots \ldots \ldots \ldots \ldots \ldots \ldots \ldots \ldots \ldots \ldots \ldots \ldots \ldots \ldots \ldots \ldots$

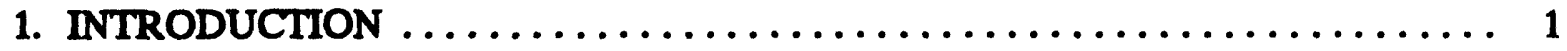

1.1 THE SCALE SYSTEM $\ldots \ldots \ldots \ldots \ldots \ldots \ldots \ldots \ldots \ldots \ldots \ldots \ldots \ldots \ldots$

1.2 THE AMPX SYSTEM $\ldots \ldots \ldots \ldots \ldots \ldots \ldots \ldots \ldots \ldots \ldots \ldots$

1.3 EXISTING MULTIGROUP CROSS-SECTION LIBRARIES FOR THE SCALE SYSTEM $\ldots \ldots \ldots \ldots \ldots \ldots \ldots \ldots \ldots \ldots \ldots \ldots \ldots \ldots \ldots$

2. CHARACTERISTICS OF THE LAW LIBRARY $\ldots \ldots \ldots \ldots \ldots \ldots \ldots \ldots \ldots$

2.1 NUCLIDES IN THE LAW LIBRARY ................. 8

2.2 RESONANCE SELF-SHIELDING THE LAW LIBRARY $\ldots \ldots \ldots \ldots \ldots 16$

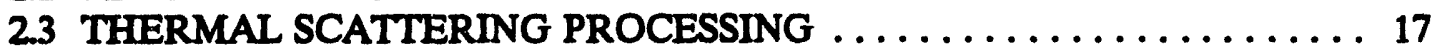

2.4 SPECIAL WEIGHTING FUNCTIONS PROVIDED TO COLLAPSE 238-GROUP LIBRARY ..................... 17

3. LIBRARY PRODUCTION PROCEDURES $\ldots \ldots \ldots \ldots \ldots \ldots \ldots \ldots \ldots \ldots$

4. LIBRARY TESTING AND CONCLUSIONS $\ldots \ldots \ldots \ldots \ldots \ldots \ldots \ldots \ldots \ldots$

4.1 HISTORY OF ENDF/B-V DATA TESTING $\ldots \ldots \ldots \ldots \ldots \ldots \ldots \ldots . \ldots \ldots$

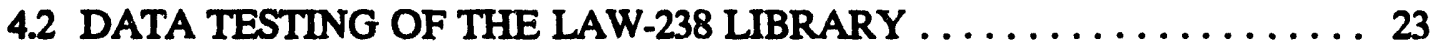

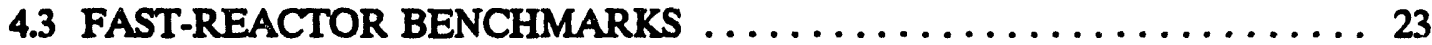

4.4 THERMAL-REACTOR BENCHMARKS $\ldots \ldots \ldots \ldots \ldots \ldots \ldots \ldots . \ldots \ldots$

4.4.1 ORNL Spheres (uranyl nitrate in water) ............. 24

4.4.2. Uranium Lattice Benchmark Testing . . . . . . . . . . . 25

4.4.3. Uranyl Fluoride $\left(\mathrm{UO}_{2} \mathrm{~F}_{2}\right) / \mathrm{H}_{2} \mathrm{O}$ Moderator $\ldots \ldots \ldots \ldots \ldots \ldots \ldots 27$

4.4.4. Homogeneous $\mathrm{Pu}-\mathrm{H}_{2} \mathrm{O}$ Assemblies ................ 29

4.4.5. Other Fast Benchmarks ................... 29

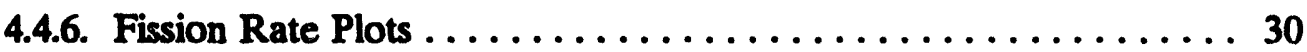

4.4.7. Calculations with ENDF/B-VI Oxygen $\ldots \ldots \ldots \ldots \ldots \ldots \ldots$

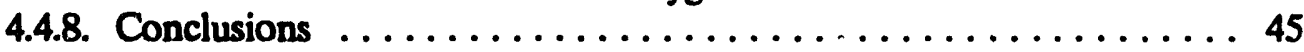

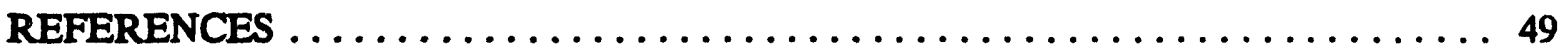

APPENDIX A NEW RESONANCE DATA $\ldots \ldots \ldots \ldots \ldots \ldots \ldots \ldots \ldots$ 


\section{LIST OF FIGURES}

Fivure

Page

1. Weighting function for 238 -group $\mathrm{LAW}$ Library $\ldots \ldots \ldots \ldots \ldots \ldots \ldots \ldots$

2. Neutron production path for most nuclides in the 238-group library . . . . . . . 21

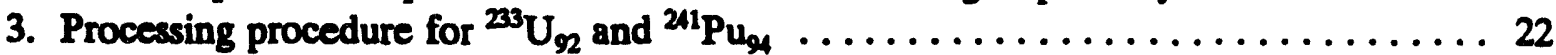

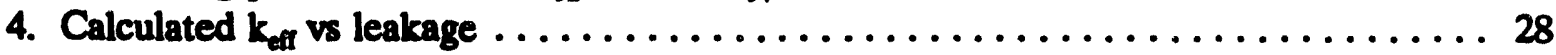

5. UH3-NISCALE-238 Ni total cross section $\ldots \ldots \ldots \ldots \ldots \ldots \ldots \ldots \ldots \ldots \ldots$

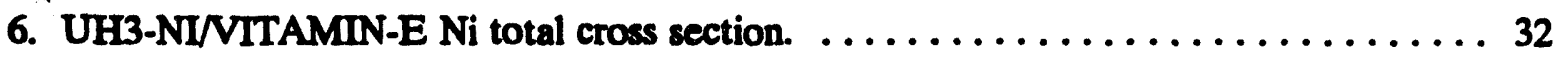

7. L11CSB fission rate for 0.001 to $10 \mathrm{eV} . \ldots \ldots \ldots \ldots \ldots \ldots \ldots \ldots \ldots \ldots \ldots$

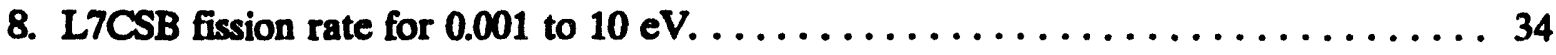

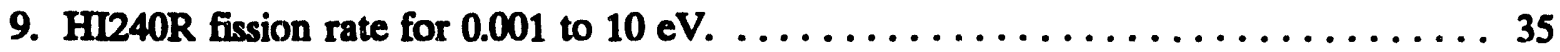

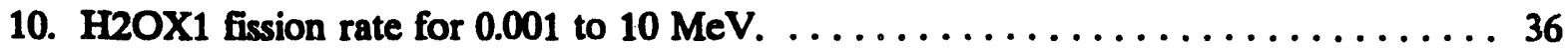

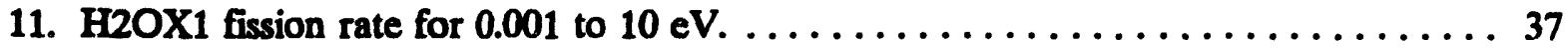

12. UH3NI fission rate for 0.001 to $10 \mathrm{MeV}, \ldots \ldots \ldots \ldots \ldots \ldots \ldots \ldots \ldots \ldots$

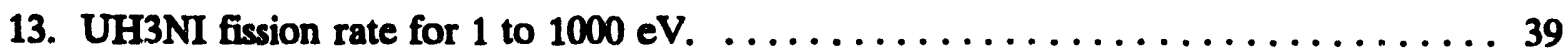

14. HISS(HUG) fission rate for 0.1 to $100 \mathrm{eV} \ldots \ldots \ldots \ldots \ldots \ldots \ldots \ldots \ldots$

15. HISS(HUG) fission rate for 1 to $100,000 \mathrm{eV} . \ldots \ldots \ldots \ldots \ldots \ldots \ldots \ldots \ldots$

16. HISS(HPG) fission rate for 0.1 to $100 \mathrm{eV} . \ldots \ldots \ldots \ldots \ldots \ldots \ldots \ldots \ldots \ldots \ldots$

17. ZPR-3/12 fission rate for 0.001 to $10 \mathrm{MeV} . \ldots \ldots \ldots \ldots \ldots \ldots \ldots \ldots \ldots \ldots$

18. ZPR-6/6A fission rate for 0.001 to $10 \mathrm{MeV} . \ldots \ldots \ldots \ldots \ldots \ldots \ldots \ldots \ldots$

19. $k_{\text {eff }}$ vs leakage with ENDF/B-VI ${ }^{16} \mathrm{O} \ldots \ldots \ldots \ldots \ldots \ldots \ldots \ldots \ldots \ldots \ldots$ 


\section{LIST OF TABLES}

Table

Page

1. Group structure for the 238 group LAW Library $\ldots \ldots \ldots \ldots \ldots \ldots \ldots \ldots$

2. Contents of 238 -group LAW Library $\ldots \ldots \ldots \ldots \ldots \ldots \ldots \ldots \ldots \ldots$

3. ENDF/B-V nuclides with $\mathrm{p}$ - and d-wave resonance parameters $\ldots \ldots \ldots \ldots \ldots \ldots$

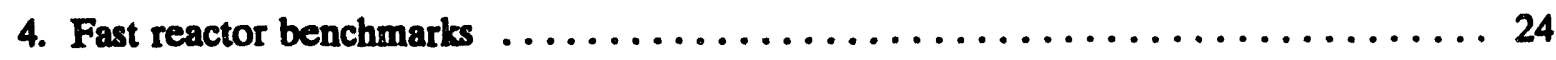

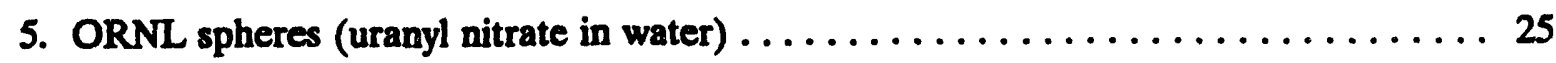

6. Uranium- $\mathrm{H}_{2} \mathrm{O}$-moderated lattices $\ldots \ldots \ldots \ldots \ldots \ldots \ldots \ldots \ldots \ldots \ldots \ldots \ldots$

7. Uranyl fluoride $\left(\mathrm{UO}_{2} \mathrm{~F}_{2}\right) / \mathrm{H}_{2} \mathrm{O}$ moderator $) \ldots \ldots \ldots \ldots \ldots \ldots \ldots \ldots \ldots \ldots \ldots$

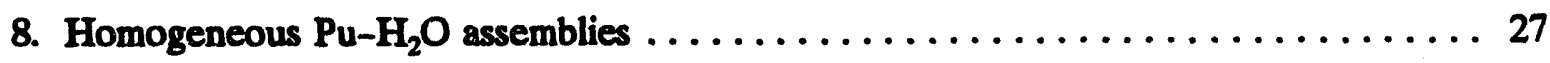

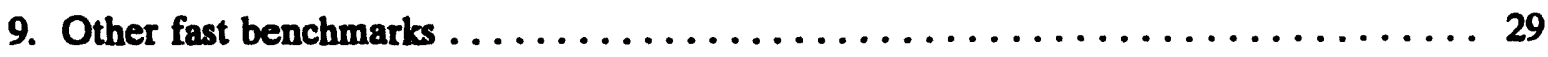

10. Thermal benchmarks with ENDF/B-VI ${ }^{16} \mathrm{O} \ldots \ldots \ldots \ldots \ldots \ldots \ldots \ldots \ldots$

11. Benchmarks for which further study is recommended $\ldots \ldots \ldots \ldots \ldots \ldots \ldots$

A.1 Comparison of results using $\mathrm{L}=0$ and $\mathrm{L}=0,1$, and 2 resonance data $\ldots \ldots \ldots 54$ 


\section{ACKNOWLEDGMENTS}

The authors are deeply appreciative for the efforts of M. C. (Mikey) Brady (now at Sandia National Laboratories), who performed the initial data testing of the LAW Library, and to Steve Bowman and Mark DeHart, who continued the testing on an additional set of calculational benchmarks.

Finally, we note that Lindy Norris performed her usual superlative job of transforming very rough drafts of a report such as this one into a readable and attractive product. 


\begin{abstract}
The 238-group LAW Library is a new multigroup neutron cross-section library based on ENDF/B-V data, with five sets of data taken from ENDF/B-VI $\left({ }^{14} \mathrm{~N}_{7},{ }^{15} \mathrm{~N}_{7},{ }^{16} \mathrm{O}_{8},{ }^{154} \mathrm{Eu}_{63}\right.$, and $\left.{ }^{155} \mathrm{Eu}_{63}\right)$. These five nuclides are included because the new evaluations are thought to be superior to those in Version V. The LAW Library contains data for over 300 materials and will be distributed by the Radiation Shielding Information Center, located at Oak Ridge National Laboratory. It was generated for use in neutronics calculations required in radioactive waste analyses, although it has equal utility in any study requiring multigroup neutron cross sections.
\end{abstract}




\section{INTRODUCTION}

In the latter part of 1988, an effort was initiated to produce a multigroup neutron crosssection library to be used in calculations associated with radioactive waste analyses. The impetus was twofold:

1. A new version of the AMPX ${ }^{1}$ system had been implemented on the IBM and CRAY computers at Oak Ridge. The new library would, therefore, be able to take advantage of extensions and improvements provided in this system.

2. The analysis of radioactive waste requires cross sections for a very large number of isotopes. The new library was to contain data for as many nuclides as could be found in the ENDF/B-V $V^{2}$ data collections. It also contains data for ${ }^{16} \mathrm{~N}_{7},{ }^{15} \mathrm{~N}_{7},{ }^{16} \mathrm{O}_{8}{ }^{154} \mathrm{Eu}_{63}$, and ${ }^{155} \mathrm{Eu}_{63}$, taken from $\mathrm{ENDF} / \mathrm{B}-\mathrm{VI}{ }^{3}$

A natural course of action would have been to start with the 227-group library that was produced several years earlier because it was based on ENDF/B-V data and already contained data for 130 materials. However, it was recognized that several improvements had been made to the processing codes, especially in the processing of resonance data. These improvements, combined with a desire to produce a library with all the traceability required by present quality assurance procedures, led to a decision to produce new values for all materials included in the library. The new library has been named the LAW Library, or Library to Analyze Radioactive Waste. Alternatively, the LAW Library is referred to as the SCALE-238 Library because of its group structure and use of SCALE nuclide identifiers.

\subsection{THE SCALE SYSTEM}

The SCALES modular code system was developed with support from the U.S. Nuclear Regulatory Commission (NRC) for use in the evaluation and study of the nuclear aspects of fuel cycle facilities and transportation packages. The system is ideal for criticality, shielding, and source term (radiation, decay heat, isotopic concentrations) analyses related to radioactive waste and spent fuel. The modules of the system can be used individually or the user can access one of the standard analysis sequences that can help facilitate the analysis.

Even though the new cross sections will be used in SCALE, they can also be output in a variety of alternative formats that are used by several major independent programs, such as DOT, ${ }^{6}$ ANISN, 7 VENTURE, ${ }^{8}$ MORSE, ${ }^{9}$ etc.

\subsection{THE AMPX SYSTEM}

The LAW Library was produced by the AMPX-77 system.' The library will be distributed in the AMPX master library format. AMPX-77 is a version of AMPX which uses FORTRAN-77 and contains the latest versions of the ever-evolving codes in the systems.

AMPX was developed for the Defense Nuclear Agency to provide an integrated capability to produce multigroup cross sections for use in various shielding and radiation effects studies. It is a modular system and, in addition to the codes that produce multigroup data, includes several routines for manipulating and further processing multigroup data, including 
codes to convert cross sections from its master library format to the formats used in the codes described in refs. 6-9.

AMPX was developed in the early 1970 s on IBM computers. Subsequently, partial implementations of the system have been made on other computing systems, such as CDC, Prime, and UNIVAC, though never in anything approaching a full implementation.

An effort was completed in October 1988 to convert the existing AMPX modules from the IBM FORTRAN-H dialect to the newer FORTRAN-77. This new version of the system was fully implemented on both IBM and CRAY systems. The bulk of the LAW Library was produced on the CRAY version of the system, although several nuclides (as described below) have been reprocessed using higher accuracy data and procedures on the IBM-3090 at Oak Ridge National Laboratory (ORNL). The CRAY version was not used because these new data were produced after retirement of the CRAY in September 1992.

The new version of AMPX has been made available to the Radiation Shielding Information Center (RSIC) located at ORNL, along with an updated User's Guide.

\subsection{EXISTINGMULTIGROUP CROSS-SECTION LIBRARIES FOR THESCALESYSTEM}

The SCALE system provides several multigroup cross-section libraries in the AMPX master library format for its neutronics calculations:

1. a 16-group library based primarily on Hansen-Roach ${ }^{10}$ data, augmented by sets produced from various versions of ENDF/B data;

2. a 123-group library formed from 99 -group GAM-II"1 ${ }^{11}$ cross sections coupled to 30-group THERMOS ${ }^{12}$ libraries produced at ORNL;

3. a 218-group library ${ }^{13}$ produced by AMPX from Version IV ENDF/B data;

4. a 27-group library produced by collapsing the 218-group library; and

5. an alternative version of the 27-group library supplemented with fission-product data.

The 16- and 123-group libraries have been used extensively and produce good results for many applications, probably because they have been adjusted to fit a variety of experiments.

The 218-group library is based on ENDF/B evaluations. One of the major tenets of ENDF is to reject adjustments that make evaluations produce "correct" results. This approach is taken to ensure that evaluations improve because of advances in the evaluation art and not because of some fortuitous (and often misunderstood) change in an associated calculational procedure. In this spirit, our ENDF/B-derived libraries are never adjusted.

The 218-group and its 27-group derivative have been benchmarked against some 400 or 500 critical experiments, including both uranium- and plutonium-fueled assemblies. Comparisons of calculations ${ }^{13}$ with both multigroup and point Monte Carlo calculations verified the processing of the library and established calculational biases that were due to inadequacies in the Version IV ENDF/B evaluations.

A 227-group ENDF/B-V library and a 27-group derivative were produced and had some benchmarking performed, although not nearly as much as the 218-group data. These libraries were produced during a period of austere support and, because of limited benchmarking, were never released to the public. 
Taking into account the differences between Versions IV and V of ENDF/B, the 227and 218-group libraries have been shown to produce results that compare favorably with other published results for the same criticals. However, note that the 27-group library, derived from the 218-group library, produces more consistent results than does the 27-group library derived from the 227-group library.

An examination of the procedures used to collapse the two libraries reveals the reason the older 27-group library gives better results.

Many of the important nuclides in the 218-group library were collapsed using a spectrum consisting of a Maxwellian spectrum below $0.12 \mathrm{eV}$, coupled to a $1 /\left(\mathrm{E}_{\sigma_{1}}\right)$ spectrum extending to $67 \mathrm{keV}$, and coupled to a fission spectrum above that. The 227-group library used a similar scheme, except that the region between $0.12 \mathrm{eV}$ and $67 \mathrm{keV}$ used a 1/E spectrum. Either of these weighting schemes is generally adequate for the fine-group libraries. As noted below, the 1/E spectrum was applied in this energy range for generating the LAW Library. Certainly for nuclides with strong resonances, all of these libraries will have additional weighting with a resonance self-shielding calculation. For the 27-group libraries, broad-group collapsing used the fine-group weighting spectra. However, since the weighting spectrum of the 218-group library contained a $1 /\left(E_{\sigma_{1}}\right)$ part, and since this is closer to a "real" flux (especially when the nuclide is important) than the 1/E variation in the 227-group library spectrum, the older 27-group library based on ENDF/B-IV produced better results.

It was probably a mistake to make it so easy (in the AMPX MALOCS module) to collapse a fine-group library using the spectrum that produced the fine-group library. The option was programmed primarily for testing collapsing procedures and not as a recommended way to make broad-group libraries. In any event, any future broad-group libraries derived from the new 238-group LAW Library will be collapsed over "real" spectra, produced from transport calculations for representative systems. (See Sect. 2.4 for a description of a set of characteristic weighting spectra derived from realistic cases.) 


\section{CHARACTERISTICS OF THE LAW LIBRARY}

The LAW Library uses the 238-group energy structure shown in Table 1. The structure contains all the boundaries of the 227-group structure. The extra group boundaries are all in the thermal range and were included for two principal reasons:

1. A boundary was added at $0.625 \mathrm{eV}$ to hit the cadmium-cutoff, thereby making it more convenient to calculate resonance integrals.

2. Several very low energy boundaries were added so that the group structure would adequately support calculations needing detailed structures at low energies, such as the Advanced Neutron Source Reactor. ${ }^{14}$ 
5

Table 1. Group structure for the 238-group LAW Library

\begin{tabular}{|c|c|c|c|c|c|c|c|}
\hline Group & $\begin{array}{l}\text { Upper } \\
\text { energy } \\
\text { boundary }\end{array}$ & Group & $\begin{array}{l}\text { Upper } \\
\text { energy } \\
\text { boundary }\end{array}$ & Group & $\begin{array}{l}\text { Upper } \\
\text { energy } \\
\text { boundary }\end{array}$ & Group & $\begin{array}{l}\text { Upper } \\
\text { energy } \\
\text { boundary }\end{array}$ \\
\hline 1 & $2.0000 \mathrm{E}+07$ & 41 & $2.7000 E+05$ & 81 & $1.8600 \mathrm{E}+02$ & 121 & $2.0000 \mathrm{E}+01$ \\
\hline 2 & $1.7333 E+07$ & 42 & $2.0000 \mathrm{E}+05$ & 82 & $1.2200 \mathrm{E}+02$ & 122 & $1.9000 \mathrm{E}+01$ \\
\hline 3 & $1.5683 E+07$ & 43 & $1.5000 E+05$ & 83 & $1.1900 \mathrm{E}+02$ & 123 & $1.8500 E+01$ \\
\hline 4 & $1.4550 \mathrm{E}+07$ & 44 & $1.2830 E+05$ & 84 & $1.1500 E+02$ & 124 & $1.7000 \mathrm{E}+01$ \\
\hline 5 & $1.3840 \mathrm{E}+07$ & 45 & $1.0000 \mathrm{E}+05$ & 85 & $1.0800 \mathrm{E}+02$ & 125 & $1.6000 \mathrm{E}+01$ \\
\hline 6 & $1.2840 E+07$ & 46 & $8.5000 E+04$ & 86 & $1.0000 \mathrm{E}+02$ & 126 & $1.5100 \mathrm{E}+01$ \\
\hline 7 & $1.0000 E+07$ & 47 & $8.2000 E+04$ & 87 & $9.0000 \mathrm{E}+01$ & 127 & $1.4400 \mathrm{E}+01$ \\
\hline 8 & 8.1873E+06 & 48 & $7.5000 \mathrm{E}+04$ & 88 & 8.2000E+01 & 128 & $1.3750 E+01$ \\
\hline 9 & $6.4340 E+06$ & 49 & $7.3000 \mathrm{E}+04$ & 89 & $8.0000 E+01$ & 129 & $1.2900 E+01$ \\
\hline 10 & $4.8000 \mathrm{E}+06$ & 50 & $6.0000 E+04$ & 90 & $7.6000 E+01$ & 130 & $1.1900 \mathrm{E}+01$ \\
\hline 11 & $4.3040 \mathrm{E}+06$ & 51 & $5.2000 E+04$ & 91 & $7.2000 E+01$ & 131 & $1.1500 E+01$ \\
\hline 12 & $3.0000 \mathrm{E}+06$ & 52 & $5.0000 E+04$ & 92 & $6.7500 \mathrm{E}+01$ & 132 & $1.0000 E+01$ \\
\hline 13 & $2.4790 E+06$ & 53 & $4.5000 E+04$ & 93 & $6.5000 E+01$ & 133 & $9.1000 E+00$ \\
\hline 14 & $2.3540 E+06$ & 54 & $3.0000 \mathrm{E}+04$ & 94 & $6.1000 E+01$ & 134 & $8.1000 E+00$ \\
\hline 15 & $1.8500 E+06$ & 55 & $2.5000 E+04$ & 95 & $5.9000 E+01$ & 135 & $7.1500 E+00$ \\
\hline 16 & $1.5000 E+06$ & 56 & $1.7000 E+04$ & 96 & $5.3400 E+01$ & 136 & $7.0000 E+00$ \\
\hline 17 & $1.4000 E+06$ & 57 & $1.3000 \mathrm{E}+04$ & 97 & $5.2000 \mathrm{E}+01$ & 137 & $6.7500 \mathrm{E}+00$ \\
\hline 18 & $1.3560 \mathrm{E}+06$ & 58 & $9.5000 \mathrm{E}+03$ & 98 & $5.0600 \mathrm{E}+01$ & 138 & $6.5000 E+\infty 0$ \\
\hline 19 & $1.3170 E+06$ & 59 & $8.0300 \mathrm{E}+03$ & 99 & $4.9200 \mathrm{E}+01$ & 139 & $6.2500 \mathrm{E}+\infty 0$ \\
\hline 20 & $1.2500 E+06$ & 60 & $6.0000 E+03$ & 100 & $4.8300 E+01$ & 140 & $6.0000 E+\infty 0$ \\
\hline 21 & $1.2000 \mathrm{E}+06$ & 61 & $39000 E+03$ & 101 & $4.7000 \mathrm{E}+01$ & 141 & $5.4000 \mathrm{E}+00$ \\
\hline 22 & $1.1000 \mathrm{E}+06$ & 62 & $3.7400 E+03$ & 102 & $4.5200 \mathrm{E}+01$ & 142 & $5.0000 \mathrm{E}+00$ \\
\hline 23 & $1.0100 E+06$ & 63 & $3.0000 E+03$ & 103 & $4.4000 \mathrm{E}+01$ & 143 & $4.7500 \mathrm{E}+\infty 0$ \\
\hline 24 & $9.2000 E+05$ & 64 & $2.5800 \mathrm{E}+03$ & 104 & $4.2400 \mathrm{E}+01$ & 144 & $4.0000 \mathrm{E}+\infty 0$ \\
\hline 25 & $9.0000 E+05$ & 65 & $2.2900 E+03$ & 105 & 4.1000E +01 & 145 & $3.7300 \mathrm{E}+\infty 0$ \\
\hline 26 & 8.7500E+05 & 66 & $2.2000 \mathrm{E}+03$ & 106 & $3.9600 \mathrm{E}+01$ & 146 & $3.5000 \mathrm{E}+00$ \\
\hline 27 & 8.6110E+05 & 67 & $1.8000 \mathrm{E}+03$ & 107 & $3.9100 \mathrm{E}+01$ & 147 & $3.1500 \mathrm{E}+00$ \\
\hline 28 & 8.2000E+05 & 68 & $1.5500 \mathrm{E}+03$ & 108 & $3.8000 \mathrm{E}+01$ & 148 & $3.0500 \mathrm{E}+00$ \\
\hline 29 & $7.5000 \mathrm{E}+05$ & 69 & $1.5000 \mathrm{E}+03$ & 109 & $3.7000 \mathrm{E}+01$ & 149 & $3.0000 \mathrm{E}+00$ \\
\hline 30 & $6.7900 \mathrm{E}+05$ & 70 & $1.1500 \mathrm{E}+03$ & 110 & $3.5500 E+01$ & 150 & $2.9700 \mathrm{E}+00$ \\
\hline 31 & $6.7000 E+05$ & 71 & $9.5000 \mathrm{E}+02$ & 111 & $3.4600 \mathrm{E}+01$ & 151 & $2.8700 \mathrm{E}+00$ \\
\hline 32 & $6.0000 E+05$ & 72 & $6.8300 \mathrm{E}+02$ & 112 & $3.3750 \mathrm{E}+01$ & 152 & $2.7700 \mathrm{E}+00$ \\
\hline 33 & $5.7300 \mathrm{E}+05$ & 73 & $6.7000 \mathrm{E}+02$ & 113 & $3.3250 \mathrm{E}+01$ & 153 & $2.6700 \mathrm{E}+00$ \\
\hline 34 & $5.5000 \mathrm{E}+05$ & 74 & $5.5000 \mathrm{E}+02$ & 114 & $3.1750 \mathrm{E}+01$ & 154 & $2.5700 \mathrm{E}+00$ \\
\hline 35 & $4.9952 \mathrm{E}+05$ & 75 & $3.0500 \mathrm{E}+02$ & 115 & $3.1250 \mathrm{E}+01$ & 155 & $2.4700 \mathrm{E}+00$ \\
\hline 36 & $4.7000 \mathrm{E}+05$ & 76 & $2.8500 \mathrm{E}+02$ & 116 & $3.0000 E+01$ & 156 & $2.3800 \mathrm{E}+00$ \\
\hline 37 & $4.4000 \mathrm{E}+05$ & 77 & $2.4000 \mathrm{E}+02$ & 117 & $2.7500 \mathrm{E}+01$ & 157 & $2.3000 \mathrm{E}+00$ \\
\hline 38 & $4.2000 \mathrm{E}+05$ & 78 & $2.1000 \mathrm{E}+02$ & 118 & $2.5000 \mathrm{E}+01$ & 158 & $2.2100 \mathrm{E}+00$ \\
\hline 39 & $4.0000 E+05$ & 79 & $2.0750 \mathrm{E}+02$ & 119 & $2.2500 \mathrm{E}+01$ & 159 & $2.1200 \mathrm{E}+\infty 0$ \\
\hline 40 & $3.3000 \mathrm{E}+05$ & 80 & $1.9250 \mathrm{E}+02$ & 120 & $2.1000 \mathrm{E}+01$ & 160 & $2.0000 \mathrm{E}+\infty 0$ \\
\hline
\end{tabular}


Table 1 (continued)

\begin{tabular}{ll|ll|ll|ll}
\hline Group & $\begin{array}{l}\text { Upper } \\
\text { energy } \\
\text { boundary }\end{array}$ & Group & $\begin{array}{c}\text { Upper } \\
\text { energy } \\
\text { boundary }\end{array}$ & Grcup & $\begin{array}{c}\text { Upper } \\
\text { energy } \\
\text { boundary }\end{array}$ & Group & $\begin{array}{c}\text { Upper } \\
\text { energy } \\
\text { boundary }\end{array}$ \\
\hline 161 & $1.9400 \mathrm{E}+00$ & 181 & $1.0900 \mathrm{E}+00$ & 201 & $6.0000 \mathrm{E}-01$ & 221 & $6.0000 \mathrm{E}-02$ \\
162 & $1.8600 \mathrm{E}+00$ & 182 & $1.0800 \mathrm{E}+00$ & 202 & $5.5000 \mathrm{E}-01$ & 222 & $5.0000 \mathrm{E}-02$ \\
163 & $1.7700 \mathrm{E}+00$ & 183 & $1.0700 \mathrm{E}+00$ & 203 & $5.0000 \mathrm{E}-01$ & 223 & $4.0000 \mathrm{E}-02$ \\
164 & $1.5800 \mathrm{E}+00$ & 184 & $1.0600 \mathrm{E}+00$ & 204 & $4.5000 \mathrm{E}-01$ & 224 & $3.0000 \mathrm{E}-02$ \\
165 & $1.5900 \mathrm{E}+00$ & 185 & $1.0500 \mathrm{E}+00$ & 205 & $4.0000 \mathrm{E}-01$ & 225 & $2.5300 \mathrm{E}-02$ \\
166 & $1.5000 \mathrm{E}+00$ & 186 & $1.0400 \mathrm{E}+00$ & 206 & $3.7500 \mathrm{E}-01$ & 226 & $1.0000 \mathrm{E}-02$ \\
167 & $1.4500 \mathrm{E}+00$ & 187 & $1.0300 \mathrm{E}+00$ & 207 & $3.5000 \mathrm{E}-01$ & 227 & $7.5000 \mathrm{E}-03$ \\
168 & $1.4000 \mathrm{E}+00$ & 188 & $1.0200 \mathrm{E}+00$ & 208 & $3.2500 \mathrm{E}-01$ & 228 & $5.0000 \mathrm{E}-03$ \\
169 & $1.3500 \mathrm{E}+00$ & 189 & $1.0100 \mathrm{E}+00$ & 209 & $3.0000 \mathrm{E}-01$ & 229 & $4.0000 \mathrm{E}-03$ \\
170 & $1.3000 \mathrm{E}+00$ & 190 & $1.000 \mathrm{E}+00$ & 210 & $2.7500 \mathrm{E}-01$ & 230 & $3.0000 \mathrm{E}-03$ \\
171 & $1.2500 \mathrm{E}+00$ & 191 & $9.7500 \mathrm{E}-01$ & 211 & $2.5000 \mathrm{E}-01$ & 231 & $2.5000 \mathrm{E}-03$ \\
172 & $1.2250 \mathrm{E}+00$ & 192 & $9.5000 \mathrm{E}-01$ & 212 & $2.2500 \mathrm{E}-01$ & 232 & $2.0000 \mathrm{E}-03$ \\
173 & $1.2000 \mathrm{E}+00$ & 193 & $9.2500 \mathrm{E}-01$ & 213 & $2.0000 \mathrm{E}-01$ & 233 & $1.5000 \mathrm{E}-03$ \\
174 & $1.1750 \mathrm{E}+00$ & 194 & $9.0000 \mathrm{E}-01$ & 214 & $1.7500 \mathrm{E}-01$ & 234 & $1.2000 \mathrm{E}-03$ \\
175 & $1.1500 \mathrm{E}+00$ & 195 & $8.5000 \mathrm{E}-01$ & 215 & $1.5000 \mathrm{E}-01$ & 235 & $1.0000 \mathrm{E}-03$ \\
176 & $1.1400 \mathrm{E}+00$ & 196 & $8.0000 \mathrm{E}-01$ & 216 & $1.2500 \mathrm{E}-01$ & 236 & $7.5000 \mathrm{E}-04$ \\
177 & $1.1300 \mathrm{E}+00$ & 197 & $7.5000 \mathrm{E}-01$ & 217 & $1.0000 \mathrm{E}-01$ & 237 & $5.0000 \mathrm{E}-04$ \\
178 & $1.1200 \mathrm{E}+00$ & 198 & $7.0000 \mathrm{E}-01$ & 218 & $9.0000 \mathrm{E}-02$ & 238 & $1.0000 \mathrm{E}-04$ \\
179 & $1.1100 \mathrm{E}+00$ & 199 & $6.5000 \mathrm{E}-01$ & 219 & $8.0000 \mathrm{E}-02$ & & $1.0000 \mathrm{E}-05$ \\
180 & $1.1000 \mathrm{E}+00$ & 200 & $6.2500 \mathrm{E}-01$ & 220 & $7.0000 \mathrm{E}-02$ & & \\
\hline
\end{tabular}

The selection criteria for the 227-group structure (and its predecessor 218-group structure) are documented in ref. 4. of

All nuclides in the 238-group LAW Library use the same weighting spectrum, consisting

1. Maxwellian spectrum (peak at $300 \mathrm{~K}$ ) from $10^{-5}$ to $0.125 \mathrm{eV}$,

2. a 1/E spectrum from $0.125 \mathrm{eV}$ to $67.4 \mathrm{keV}$,

3. a fission spectrum (effective temperature at $1.273 \mathrm{MeV}$ ) from $67.4 \mathrm{keV}$ to $10 \mathrm{MeV}$, and

4. a 1/E spectrum from $10 \mathrm{MeV}$ to $20 \mathrm{MeV}$.

A plot of this spectrum is shown in Fig. 1. 


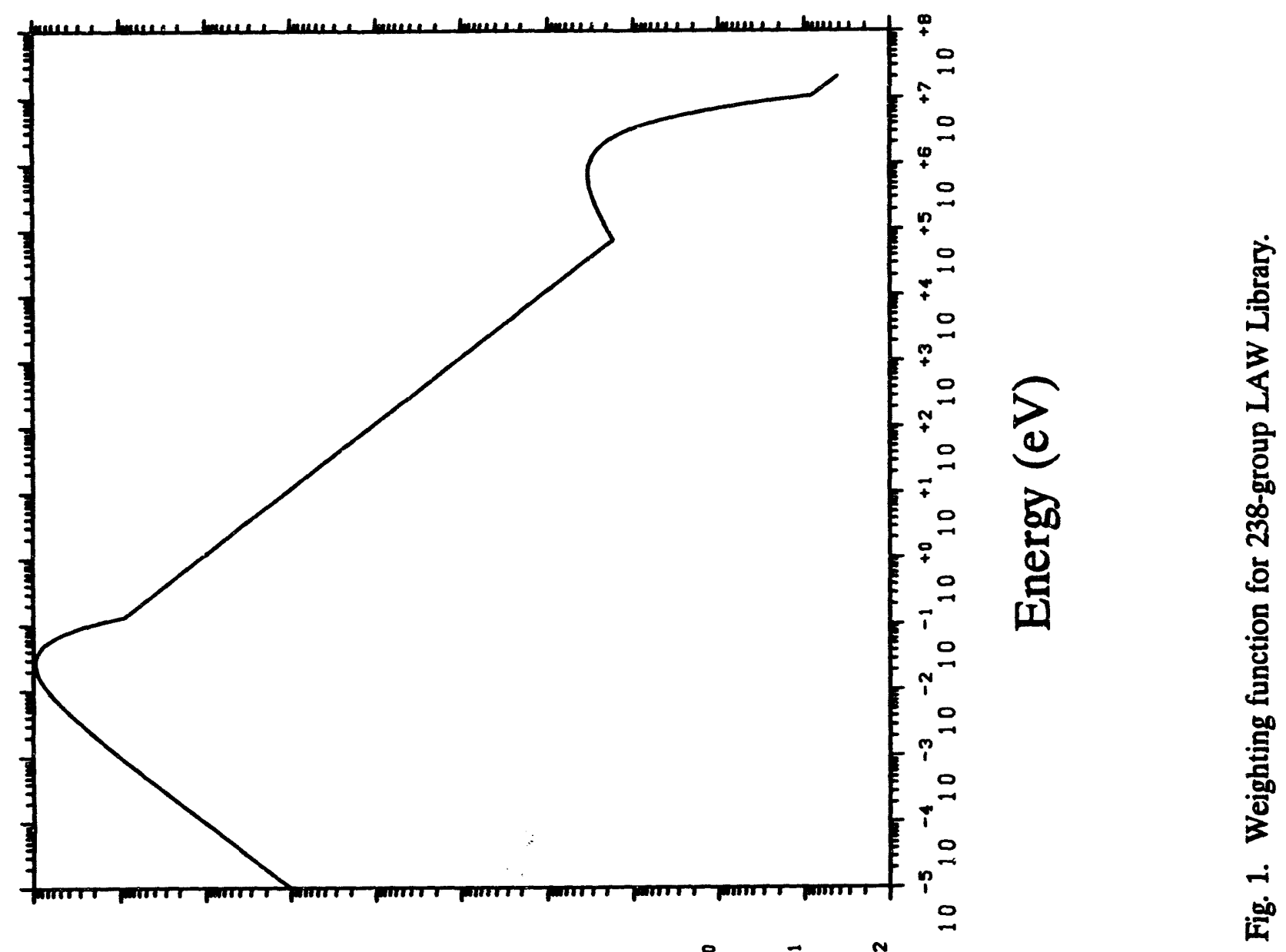

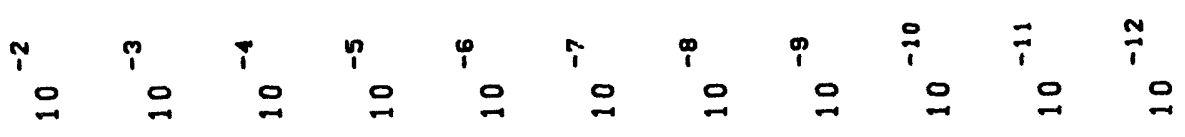

Кริมวนว ไฺนก เวd xnIH 
Even though the choice of the parameters that define this weighting spectra is somewhat arbitrary, the 238-group mesh is fine enough to help ensure that the library it produces is suitable for the class of problems it is intended to solve.

All nuclides use a $P_{5}$ Legendre expansion to fit the elastic and discrete level inelastic scattering processes, thereby making the library suitable for both reactor and shielding applications. $\mathbf{A ~}_{\mathbf{3}}$ fit was used for thermal-scattering matrices. All other scattering processes use $\mathbf{P}_{0}$ fits.

\section{NUCLIDES IN THE LAW LIBRARY}

Prior to initiating the production of the LAW Library, a task was completed to combine all complete ENDF/B evaluations from the ENDF/B-V General-Purpose, Fission Product, and Actinide files. The combined file contained about 300 sets of data. It also contains sets of data from the ENDF/B-VI file for ${ }^{14} \mathrm{~N}_{7},{ }^{15} \mathrm{~N}_{7},{ }^{16} \mathrm{O}_{8},{ }^{154} \mathrm{Eu}_{63}$, and ${ }^{155} \mathrm{Eu}_{63}$.

The contents of the LAW Library are shown in Table 2. Note that the common ZA (atomic number $\mathbf{Z}$, atomic mass $\mathrm{A}$ ) notation is used to identify cross-section sets, whenever possible. Nuclides with MA T-numbers (the ENDF/B nuclide identifier) in the 1000 range were taken from the General-Purpose file, while those from the Actinide file are in the $\mathbf{8 0 0 0}$ range, and those from the Fission Product File are in the 9000 range. The "MOD" is the revision number for the set of data; the "DATE" is the evaluation date. The cryptic notation under "RESONANCE DATA" tells what kind of resonance data, if any, are contained in the ENDF file. For example, SL17 indicates the evaluation contains 17 single-level Breit-Wigner resonances, ML17 would indicate 17 multilevel Breit-Wigner resonances, and UN indicates the evaluation has unresolved resonance data. In the case of ${ }^{233} \mathrm{U}$ and ${ }^{2 A 1} \mathrm{Pu}$, the $\mathrm{AA}$ indicates the evaluation uses the Adler-Adler formalism. A notation of BF implies this nuclide has Bondarenko factors for all energy groups.

In addition to the data on the combined ENDF file, a small number of materials require thermal-scattering law data that exist on a separate ENDF/B file. Separate sets of hydrogen were made for consideration of hydrogen bound in a water molecule, hydrogen bound in a polyethylene molecule, hydrogen bound in benzene, and hydrogen as a free gas. Deuterium sets are available for deuterium bound in $\mathrm{D}_{2} \mathrm{O}$ and for the free-gas case. A set of beryllium data considers the thermal effects existing in beryllium metal. Carbon cross sections are given in one set that has thermal data based on thermal graphite data and another based on the free-gas model. In the case of graphite, the set of cross sections uses a new graphite evaluation ${ }^{15}$ performed by J.-P. Renier, who noted that the previous evaluation, based on calculations performed by the GASKET ${ }^{16}$ code, although based on input data that are thought to be reasonable, were not converged to a reasonable level.

A special set, identified by 900, contains dose factors based on the ANSI/ANS 6.1.1-1977 standard. Dose factors based on the more recent ANSI/ANS 6.1.1-1991 standard are also included and identified by 9031 . 
Taüle 2. Contents of 238-group LAW Library

\begin{tabular}{|c|c|c|c|c|c|c|}
\hline Nuctide & $\begin{array}{c}\text { LAW } \\
\text { identifier }\end{array}$ & $\begin{array}{l}\text { ENDF } \\
\text { MAT }\end{array}$ & MOD & $\begin{array}{l}\text { Eval. } \\
\text { date }\end{array}$ & $\begin{array}{l}\text { Resonance } \\
\text { data }\end{array}$ & $\begin{array}{c}\text { Thermal } \\
\text { MAT }\end{array}$ \\
\hline 47-A\&-107 & 47107 & 1407 & 2 & Jun 1983 & ML74 & \\
\hline 47-Ag-109 & 47109 & 1409 & 2 & Jun 1983 & ML83 & \\
\hline 47-Ag-111 & 47111 & 9415 & 1 & Dec 1979 & & \\
\hline 13-AI-27 & 13027 & 1313 & 1 & Dec 1973 & $\mathbf{B F}$ & \\
\hline 95-Am-241 & 95241 & 1361 & 2 & Apr 1978 & SL66,UN & \\
\hline 95-Am-242 & 95242 & 8542 & 1 & Aug 1975 & SL82,UN & \\
\hline 95-Am-242m & 95601 & 1369 & 1 & Apr 1978 & SL6,UN & \\
\hline 95-Am-243 & 95243 & 1363 & 2 & Apr 1978 & SL220,UN & \\
\hline 33-As-75 & 33075 & 9071 & 1 & Apr 1974 & SL32 & \\
\hline 79-Au-197 & 79197 & 1379 & 3 & Feb 1977 & ML263 & \\
\hline 5-B-10 & 5010 & 1305 & 1 & Dec 1976 & & \\
\hline 5-B-11 & 5011 & 1160 & 1 & Sep 1971 & & \\
\hline 56-Ba-134 & 56134 & 9684 & 1 & Apr 1974 & SL8 & \\
\hline $56-\mathrm{Ba}-135$ & 56135 & 9685 & 1 & Apr 1974 & SL13 & \\
\hline 56-Ba-136 & 56136 & 9687 & 1 & Apr 1974 & SL3 & \\
\hline 56-Ba-137 & 56137 & 9689 & 1 & Apr 1974 & SL8 & \\
\hline 56-Ba-138 & 56138 & 1353 & 1 & Aug 1978 & & \\
\hline 56-Ba-140 & 56140 & 9693 & 1 & Dec 1979 & & \\
\hline 4-Be-9 & 4009 & 1304 & 2 & Oct 1976 & & \\
\hline Be-metal & 4309 & 1304 & 2 & & & 1064 \\
\hline 83-Bi-209 & 83209 & 1375 & 1 & Apr 1980 & & \\
\hline 97-Bk-249 & 97249 & 8749 & 1 & Jul 1976 & SL91,UN & \\
\hline 35-Br-79 & 35079 & 9113 & 1 & Apr 1974 & SL13 & \\
\hline $35-\mathrm{Br}-81$ & 35081 & 9117 & 1 & Apr 1974 & SL5 & \\
\hline $6-\mathrm{C}$ & 6012 & 1306 & 2 & Dec 1973 & & \\
\hline Graphite & 6000 & 1306 & 2 & & & 1065 \\
\hline $20-\dot{C a}$ & 20000 & 1320 & 3 & Aug 1971 & & \\
\hline 48-Cd & 48000 & 1281 & 1 & May 1974 & & \\
\hline 48-Cd-106 & 48106 & 9440 & 2 & Feb 1980 & & \\
\hline $48-C d-108$ & 48108 & 9442 & 1 & Apr 1974 & & \\
\hline $48-C d-110$ & 48110 & 9444 & 1 & Apr 1974 & SL68 & \\
\hline 48-Cd-111 & 48111 & 9445 & 1 & Apr 1974 & SL125 & \\
\hline $48-\mathrm{Cd}-112$ & 48112 & 9447 & 1 & Apr 1974 & SL55 & \\
\hline 48-Cd-113 & 48113 & 1318 & 1 & Nov 1978 & SL12,UN & \\
\hline $48-C d-114$ & 48114 & 9450 & 1 & Apr 1974 & SL33 & \\
\hline $48-C d-115$ & 48601 & 9452 & 1 & Dec 1979 & & \\
\hline $48-C d-116$ & 48116 & 9453 & 1 & Apr 1974 & SL12 & \\
\hline $58-C e-140$ & 58140 & 9724 & 1 & Apr 1974 & & \\
\hline 58-Ce-141 & 58141 & 9725 & 1 & Dec 1979 & & \\
\hline $58-\mathrm{Ce}-142$ & 58142 & 9726 & 1 & Apr 1974 & & \\
\hline $58-C e-143$ & 58143 & 9727 & 1 & Dec 1979 & & \\
\hline $58-C e-144$ & 58144 & 9728 & 1 & Dec 1979 & & \\
\hline
\end{tabular}


10

Table 2. (continued)

\begin{tabular}{|c|c|c|c|c|c|c|}
\hline Nuclide & $\begin{array}{c}\text { LAW } \\
\text { identifier }\end{array}$ & $\begin{array}{l}\text { ENDF } \\
\text { MAT }\end{array}$ & MOD & $\begin{array}{l}\text { Eval. } \\
\text { date }\end{array}$ & $\begin{array}{l}\text { Resonance } \\
\text { data }\end{array}$ & $\begin{array}{l}\text { Thermal } \\
\text { MAT }\end{array}$ \\
\hline $\begin{array}{l}98-C F-249 \\
98-C F-250\end{array}$ & 98249 & 8849 & 1 & Jul 1976 & SL53,UN & \\
\hline $\begin{array}{l}98-C f-250 \\
98-C f-251\end{array}$ & 98250 & 8850 & 1 & Jul 1976 & SL20,UN & \\
\hline $\begin{array}{l}98-C f-251 \\
98-C f-252\end{array}$ & 98251 & 8851 & 1 & Jul 1976 & SL20,UN & \\
\hline $\begin{array}{l}98-C f-252 \\
98-C f-253\end{array}$ & 98252 & 8852 & 1 & Jul 1976 & SL21,UN & \\
\hline $\begin{array}{l}98-\mathrm{Cf}-253 \\
17-\mathrm{Cl}\end{array}$ & 98253 & 8853 & 1 & Dec 1975 & SL120,UN & \\
\hline 17-CI & 17000 & 1149 & 1 & Feb 1967 & & \\
\hline $\begin{array}{l}96-\mathrm{Cm}-241 \\
96-\mathrm{Cm}-242\end{array}$ & 96241 & 8641 & 1 & Apr 1978 & & \\
\hline $\begin{array}{l}96-\mathrm{Cm}-242 \\
96-\mathrm{Cm}-243\end{array}$ & 96242 & 8642 & 1 & Apr 1978 & SL21,UN & \\
\hline $96-\mathrm{Cm}-243$ & 96243 & 1343 & 1 & Apr 1978 & SL16,UN & \\
\hline $96-\mathrm{Cm}-244$ & 96244 & 1344 & 2 & Apr 1978 & SL38,UN & \\
\hline $96-\mathrm{Cm}-245$ & 96245 & 1345 & 2 & $\operatorname{Jan} 1979$ & SL39,UN & \\
\hline $96-\mathrm{Cm}-246$ & 96246 & 1346 & 1 & Jul 1976 & SL10,UN & \\
\hline $96-\mathrm{Cm}-247$ & 96247 & 8647 & 1 & Jul 1976 & SL35,UN & \\
\hline $96-\mathrm{Cm}-248$ & 96248 & 8648 & 1 & Apr 1978 & SLA6,UN & \\
\hline $27-C 0-59$ & 27059 & 1327 & 3 & Jun 1977 & ML180 & \\
\hline $24-C$ & 24000 & 1324 & 2 & Dec 1977 & SL183 & \\
\hline $24-\operatorname{Cr}\left(1 / E^{*} \sigma_{0}\right)$ & 24301 & 1324 & 2 & Dec 1977 & SL183 & \\
\hline $24-C r\left(1 / E^{*} \sigma_{i}(\mathrm{SS} 304)\right)$ & 24304 & 1324 & 2 & Dec 1977 & SL183 & \\
\hline $55-C s-133$ & 55133 & 1355 & 1 & Nov 1978 & SL123 & \\
\hline $55-C 5-134$ & 55134 & 9663 & 1 & Dec 1979 & & \\
\hline $55-C 2-135$ & 55135 & 9665 & 1 & Dec 1979 & & \\
\hline $55-C 3-136$ & 55136 & 9667 & 1 & Dec 1979 & SL7 & \\
\hline $55-C 2-137$ & 55137 & 9669 & 1 & Dec 1979 & & \\
\hline 29-Cu & 29000 & 1329 & 1 & Dec 1973 & MLA8 & \\
\hline $\begin{array}{l}\text { 1-D-2 } \\
\text { Deuterium }\end{array}$ & 1802 & 1302 & 2 & Nov 1967 & & 1004 \\
\hline $\begin{array}{l}\text { Deuterium } \\
66-\text { Dy-160 }\end{array}$ & $\begin{array}{l}1002 \\
66160\end{array}$ & $\begin{array}{l}1302 \\
9864\end{array}$ & $\begin{array}{l}2 \\
1\end{array}$ & Apr 1974 & SL3 & \\
\hline 66-Dy-161 & 66161 & 9865 & 1 & Apr 1974 & SL27 & \\
\hline 66-Dy-162 & 66162 & 9866 & 1 & Apr 1974 & SL8 & \\
\hline 66-Dy-163 & 66163 & 9867 & 1 & Apr 1974 & SL60 & \\
\hline 66-Dy-164 & 66164 & 1031 & 1 & Jun 1967 & SL2,UN & \\
\hline 68-Er-166 & 68166 & 9875 & 1 & Apr 1974 & SL51 & \\
\hline 68-Er-167 & 68167 & 9876 & 1 & Apr 1974 & SL32 & \\
\hline 99-Es-253 & 99253 & 8953 & 1 & Jul 1976 & SL27,UN & \\
\hline 63-Eu & 63000 & 1463 & 1 & Aug 1981 & ML164 & \\
\hline 63-Eu-151 & 63151 & 1357 & 1 & Dec 1977 & SL92,UN & \\
\hline 63-Eu-152 & 63152 & 1292 & 2 & Dec 1973 & SL93,UN & \\
\hline 63-Eu-153 & 63153 & 1359 & 1 & Feb 1978 & ML72,UN & \\
\hline 63-Eu-154 & 63154 & Version & f ENDF/B & & & \\
\hline 63-Eu-154 & 631541 & 1293 & 1 & Dec 1973 & SL72,UN & \\
\hline 63-Eu-155 & 63155 & Version & ff ENDF/B & & & \\
\hline 63-Eu-155 & 631551 & 9832 & 1 & Dec 1979 & & \\
\hline
\end{tabular}


Table 2. (continued)

\begin{tabular}{|c|c|c|c|c|c|c|}
\hline Nuclide & $\begin{array}{c}\text { LAW } \\
\text { identifier }\end{array}$ & $\begin{array}{l}\text { ENDF } \\
\text { MAT }\end{array}$ & MOD & $\begin{array}{l}\text { Eval. } \\
\text { date }\end{array}$ & $\begin{array}{c}\text { Resonance } \\
\text { data }\end{array}$ & $\begin{array}{c}\text { Thermal } \\
\text { MAT }\end{array}$ \\
\hline 63-Eu-156 & 63156 & 9833 & 1 & Dec 1979 & & \\
\hline 63-Eu-157 & 63157 & 9834 & 1 & Feb 1980 & & \\
\hline 9-F-19 & 9019 & 1309 & 3 & Jul 1974 & $\mathbf{B F}$ & \\
\hline 26-Fe & 26000 & 1326 & 3 & Oct 1977 & ML314 & \\
\hline 26-Fe $\left(1 / E^{*} \sigma_{0}\right)$ & 26301 & 1326 & 3 & Oct 1977 & ML314 & \\
\hline $26-\mathrm{Fe}\left(1 / \mathrm{E}^{*} \sigma_{1}(\mathrm{SS} 304)\right)$ & 26301 & 1326 & 3 & Oct 1977 & ML314 & \\
\hline 31-Ga-11 & 31000 & 1358 & 1 & May 1980 & & \\
\hline 64-Gd-152 & 64152 & 1362 & 2 & Jan 1977 & ML18 & \\
\hline 64-Gd-154 & 64154 & 1364 & 1 & Jan 1977 & MLA9 & \\
\hline 64-Gd-155 & 64155 & 1365 & 1 & Jan 1977 & ML92 & \\
\hline 64-Gd-156 & 64156 & 1366 & 2 & $\operatorname{Jan} 1977$ & ML30,UN & \\
\hline 64-Gd-157 & 64157 & 1367 & 1 & $\operatorname{Jan} 1977$ & ML56 & \\
\hline 64-Gd-158 & 64158 & 1368 & 1 & Jan 1977 & ML93,UN & \\
\hline $64-6 d-160$ & 64160 & 1370 & 1 & Jan 1977 & MLA4 & \\
\hline 32-Ge-72 & 32072 & 9050 & 1 & Apr 1974 & SL8 & \\
\hline 32-Ge-73 & 32073 & 9051 & 1 & Apr 1974 & SL10 & \\
\hline 32-Ge-74 & 32074 & 9053 & 1 & Apr 1974 & SL3 & \\
\hline $32-G e-76$ & 32076 & 9056 & 1 & Apr 1974 & SLA & \\
\hline 1-H-1 & 1801 & 1301 & 1 & Aug 1970 & & \\
\hline $\mathrm{H}$ in $\mathrm{C6H6}$ & 1901 & 1301 & 1 & & & 1095 \\
\hline $\mathrm{H}$ in $\mathrm{CH} 2$ & 2801 & 1301 & 1 & & & 1114 \\
\hline $\mathrm{H}$ in $\mathrm{H} 2 \mathrm{O}$ & 1001 & 1301 & 1 & & & 1002 \\
\hline 2-He-3 & 2003 & 1146 & 1 & Jun 1968 & & \\
\hline 2-He-4 & 2004 & 1270 & 0 & Oct 1973 & & \\
\hline 72-Hf & 72000 & 1372 & 1 & Apr 1976 & SL236 & \\
\hline 72-Hf-174 & 72174 & 1374 & 1 & Apr 1976 & SL10,UN & \\
\hline 72-Hf-176 & 72176 & 1376 & 1 & Apr 1976 & SL22,UN & \\
\hline 72-Hf-177 & 72177 & 1377 & 1 & Apr 1976 & SL99,UN & \\
\hline 72-Hf-178 & 72178 & 1378 & 1 & Apr 1976 & SL25,UN & \\
\hline 72-Hf-179 & 72179 & 1383 & 1 & Apr 1976 & SL49,UN & \\
\hline 72-Hf-180 & 72180 & 1384 & 1 & Apr 1976 & SL31,UN & \\
\hline 67-Ho-165 & 67165 & 9872 & 1 & Apr 1974 & SL29 & \\
\hline $53-I-127$ & 53127 & 9606 & 1 & Feb 1980 & SL79 & \\
\hline 53-I-129 & 53129 & 9608 & 1 & Feb 1980 & SLS & \\
\hline $53-I-130$ & 53130 & 9609 & 1 & Dec 1979 & & \\
\hline 53-I-131 & 53131 & 9611 & 1 & Dec 1979 & & \\
\hline 53-I-135 & 53135 & 9618 & 1 & Dec 1979 & & \\
\hline 49-In-113 & 49113 & 9473 & 1 & Apr 1974 & SL9 & \\
\hline 49-In-115 & 49115 & .9477 & 1 & Dec 1979 & SL91 & \\
\hline $19-\mathrm{K}$ & 19000 & 1150 & 1 & Feb 1967 & & \\
\hline $36-\mathrm{Kr}-78$ & 36078 & 1330 & 1 & Apr 1978 & SLA & \\
\hline $36-\mathrm{Kr} r-80$ & 36080 & 1331 & 1 & Apr 1978 & SL5 & \\
\hline $36-\mathrm{Kr}-82$ & 36082 & 1332 & 1 & Apr 1978 & SL2 & \\
\hline $36-K r-83$ & 36083 & 1333 & 1 & Apr 1978 & SL3 3 & \\
\hline
\end{tabular}


Table 2. (continued)

\begin{tabular}{|c|c|c|c|c|c|c|}
\hline Nuctide & $\begin{array}{c}\text { LAW } \\
\text { identifier }\end{array}$ & $\begin{array}{l}\text { ENDF } \\
\text { MAT }\end{array}$ & MOD & $\begin{array}{l}\text { Eval. } \\
\text { date }\end{array}$ & $\begin{array}{l}\text { Resonance } \\
\text { data }\end{array}$ & $\begin{array}{c}\text { Thermal } \\
\text { MAT }\end{array}$ \\
\hline $36-X^{2}-84$ & 36084 & 1334 & 1 & Apr 1978 & SL3 & \\
\hline $36-\mathrm{Kr}-85$ & 36085 & 9145 & 1 & Dec 1979 & & \\
\hline $36-Z^{\prime} r-86$ & 36086 & 1336 & 1 & Jul 1972 & SL1 & \\
\hline 57-La-139 & 57139 & 9707 & 1 & Feb 1980 & SL69 & \\
\hline 57.La-140 & 57140 & 9708 & 1 & Dec 1979 & & \\
\hline 3-Li-6 & 3006 & 1303 & 1 & Sep 1977 & & \\
\hline 3-Li-7 & 3007 & 1397 & 1 & Dec 1981 & $\mathbf{B F}$ & \\
\hline 71-Lu-175 & 71175 & 1032 & 1 & Jun 1967 & SL17,UN & \\
\hline 71-Lu-176 & 71176 & 1033 & 1 & Jun 1967 & SL21,UN & \\
\hline $12-\mathrm{Mg}_{8}$ & 12000 & 1312 & 1 & Feb 1978 & & \\
\hline 25-Mn-55 & 25055 & 1325 & 2 & Mar 1977 & ML141 & \\
\hline 42-Mo & 42000 & 1321 & 1 & Feb 1979 & SL46 & \\
\hline 42-Mo-92 & 42092 & 9278 & 1 & Feb 1980 & SL28 & \\
\hline 42-Mo-94 & 42094 & 9281 & 1 & Feb 1980 & SL10 & \\
\hline 42-Mo-95 & 42095 & 9282 & 1 & Feb 1980 & SL55 & \\
\hline 42-Mo-96 & 42096 & 9283 & 1 & Feb 1980 & SL15 & \\
\hline 42-Mo-97 & 42097 & 9284 & 1 & Feb 1980 & SL64 & \\
\hline 42-Mo-98 & 42098 & 9285 & 1 & Feb 1980 & SL22 & \\
\hline 42-Mo-99 & 42099 & 9286 & 1 & Dec 1979 & & \\
\hline $42-\mathrm{MO}-100$ & 42100 & 9287 & 1 & Feb 1980 & SL17 & \\
\hline 7-N-14 & $7014 i$ & 1275 & 2 & Jul 1973 & & \\
\hline 7-N-14 & 7014 & \multicolumn{5}{|c|}{ Version 6 of ENDF/B } \\
\hline $7-N-15$ & 70151 & 1307 & 1 & Mar 1977 & & \\
\hline $7-N-15$ & 7015 & \multicolumn{5}{|c|}{ Version 6 of ENDF/B } \\
\hline 11-Na-23 & 11023 & 1311 & 3 & Dec 1977 & ML23 & \\
\hline $41-\mathrm{Nb}-93$ & 41093 & 1189 & 1 & May 1974 & SL218,UN & \\
\hline 41-Nb-94 & 41094 & 9251 & 1 & Dec 1979 & SL2 & \\
\hline 41-Nb-95 & 41095 & 9253 & 1 & Dec 1979 & & \\
\hline $60-N d-142$ & 60142 & 9763 & 1 & Apr 1974 & SL17 & \\
\hline $60-N d-143$ & 60143 & 9764 & 1 & Feb 1980 & SL18 & \\
\hline $60-\mathrm{Nd}-144$ & 60144 & 9765 & 1 & Feb 1980 & SL19 & \\
\hline $60-\mathrm{Nd}-145$ & 60145 & 9766 & 1 & Feb 1980 & SL79 & \\
\hline $60-\mathrm{Nd}-146$ & 60146 & 9767 & 1 & Feb 1980 & SL18 & \\
\hline $60-\mathrm{Nd}-147$ & 60147 & 9768 & 1 & Dec 1979 & & \\
\hline $60-\mathrm{Nd}-148$ & 60148 & 9769 & 1 & Feb 1980 & SL12 & \\
\hline $60-\mathrm{Nd}-150$ & 60150 & 9771 & 1 & Feb 1980 & SL15 & \\
\hline 28-Ni & 28000 & 1328 & 2 & Mar 1977 & SL294 & \\
\hline $28-N i\left(1 / E^{*} \sigma\right)$ & 28301 & 1328 & 2 & Mar 1977 & SL294 & \\
\hline $28-N i\left(1 / E * \sigma_{l}(S S 304)\right)$ & 28304 & 1328 & 2 & Mar 1977 & SL294 & \\
\hline 93-Np-237 & $\$ 3237$ & 1337 & 2 & Apr 1978 & SL169,UN & \\
\hline 93-Np-238 & 93238 & 8338 & 1 & Aug 1975 & SL95,UN & \\
\hline $8-0.16$ & 80161 & 1276 & 2 & Aug 1973 & & \\
\hline $8-0-16$ & 8016 & \multicolumn{5}{|c|}{ Version 6 of ENDF/B } \\
\hline $8-0-17$ & 8017 & 1317 & 1 & Jan 1978 & & \\
\hline
\end{tabular}


Table 2. (continued)

\begin{tabular}{|c|c|c|c|c|c|c|}
\hline Nuclide & $\begin{array}{c}\text { LAW } \\
\text { identifier }\end{array}$ & $\begin{array}{l}\text { ENDF } \\
\text { MAT }\end{array}$ & MOD & $\begin{array}{l}\text { Eval. } \\
\text { date }\end{array}$ & $\begin{array}{l}\text { Resonance } \\
\text { data }\end{array}$ & $\begin{array}{c}\text { Thermal } \\
\text { MAT }\end{array}$ \\
\hline 15-P-31 & 15031 & 1315 & 1 & Oet 1977 & & \\
\hline 91-Pa-231 & 91231 & 8131 & 1 & Nov 1977 & SL31,UN & \\
\hline 91-Pa-233 & 91233 & 1391 & 2 & May 1978 & SL34,UN & \\
\hline 82.Pb & 82000 & 1382 & 2 & Jul 1971 & & \\
\hline 46-Pd-102 & 46102 & 9379 & 2 & Feb 1980 & & \\
\hline 46.Pd-104 & 46104 & 9381 & 1 & Feb 1980 & SL1 & \\
\hline 46-Pd-105 & 46105 & 9382 & 1 & Feb 1980 & SL9 & \\
\hline 46-Pd-106 & 46106 & 9383 & 1 & Feb 1980 & SL1 & \\
\hline 46-Pd-107 & 46107 & 9384 & 1 & Feb 1980 & & \\
\hline 46-Pd-108 & 46108 & 9386 & 1 & Feb 1980 & SL3 & \\
\hline 46-Pd-110 & 46110 & 9389 & 1 & Feb 1980 & & \\
\hline 61-Pm-147 & 61147 & 9783 & 1 & Feb 1980 & SL14 & \\
\hline 61-Pm-148 & 61148 & 9784 & 1 & Dec 1979 & & \\
\hline 61-Pm-148m & 61601 & 9785 & 1 & Dec 1979 & SL1 & \\
\hline 61-Pm-149 & 61149 & 9786 & 1 & Dec 1979 & & \\
\hline 61-Pm-151 & 61151 & 9788 & 1 & Dec 1979 & & \\
\hline 59-Pr-141 & 59141 & 9742 & 1 & Feb 1980 & SL15 & \\
\hline 59-Pr-142 & 59142 & 9743 & 1 & Dec 1979 & & \\
\hline 59-Pr-143 & 59143 & 9745 & 1 & Dec 1979 & & \\
\hline 94-Pu-236 & 94236 & 8436 & 1 & Apr 1978 & SL21,UN & \\
\hline 94-Pu-237 & 94237 & 8437 & 1 & Apr 1978 & & \\
\hline 94-Pu-238 & 94238 & 1338 & 3 & Apr 1978 & SL16,UN & \\
\hline 94-Pu-239 & 94239 & 1399 & 2 & & SL128,UN & \\
\hline 94-Pu-240 & 94240 & 1380 & 3 & Apr 1977 & ML201,UN & \\
\hline 94-Pu-241 & 94241 & 1381 & 2 & Oct 1977 & AA83,UN & \\
\hline 94-Pu-242 & 94242 & 1342 & 2 & Oct 1978 & SL68,UN & \\
\hline 94-Pu-243 & 94243 & 8443 & 1 & Jul 1976 & SLA1,UN & \\
\hline 94-Pu-244 & 94244 & 8444 & 1 & Apr 1978 & SL21,UN & \\
\hline 37-Rb-85 & 37085 & 1360 & 1 & Oct 1979 & ML88 & \\
\hline $37-\mathbf{R b}-86$ & 37086 & 9161 & 1 & Dec 1979 & & \\
\hline 37-Rb-87 & 37087 & 1341 & 1 & Oct 1979 & ML15 & \\
\hline 75-Re-185 & 75185 & 1083 & 1 & $\operatorname{Jan} 1968$ & SL30,UN & \\
\hline 75-Re-187 & 75187 & 1084 & 1 & $\operatorname{Jan} 1968$ & SL25,UN & \\
\hline 45-Rh-103 & 45103 & 1310 & 1 & Nov 1978 & SL119,UN & \\
\hline 45-Rh-105 & 45105 & 9355 & 2 & Dec 1979 & & \\
\hline 44-Ru-96 & 44096 & 9325 & 2 & Feb 1980 & & \\
\hline 44-Ru-98 & 44098 & 9327 & 2 & Feb 1980 & & \\
\hline 44Ru-99 & 44099 & 9328 & 1 & Apr 1974 & SL5 & \\
\hline $44-R u-100$ & 44100 & 9329 & 1 & Feb 1980 & SLI & \\
\hline 44-Ru-101 & 44101 & 9330 & 1 & Feb 1980 & SL7 & \\
\hline 44-Ru-102 & 44102 & 9331 & 1 & Feb 1980 & SL3 & \\
\hline 44-Ru-103 & 44103 & 9332 & 1 & Dec 1979 & & \\
\hline 44-Ru-104 & 44104 & 9333 & 1 & Feb 1980 & SLA & \\
\hline 44-Ru-105 & 44105 & 9334 & 1 & Dec 1979 & & \\
\hline 44-Ru-106 & 44106 & 9335 & 1 & Dec 1979 & & \\
\hline $16-5$ & 16000 & 1347 & 1 & Apr 1979 & ML118 & \\
\hline $16-5-32$ & 16032 & 1316 & 1 & Oet 1977 & & \\
\hline
\end{tabular}


Table 2. (continued)

\begin{tabular}{|c|c|c|c|c|c|c|}
\hline Nuclide & $\begin{array}{c}\text { LAW } \\
\text { identifier }\end{array}$ & $\begin{array}{l}\text { ENDF } \\
\text { MAT }\end{array}$ & MOD & $\begin{array}{l}\text { Eval. } \\
\text { date }\end{array}$ & $\begin{array}{l}\text { Resonance } \\
\text { data }\end{array}$ & $\begin{array}{c}\text { Thermal } \\
\text { MAT }\end{array}$ \\
\hline $51-5 b-121$ & 51121 & 9548 & 1 & Feb 1980 & SL82 & \\
\hline $51-5 b-123$ & 51123 & 9551 & 1 & Feb 1980 & SL63 & \\
\hline S1-Sb-124 & 51124 & 9552 & 1 & Dec 1979 & & \\
\hline $51-5 b-125$ & 51125 & 9555 & 1 & Dec 1979 & & \\
\hline $51-5 b-126$ & 51126 & 9556 & 1 & Dec 1979 & & \\
\hline $34-5 e-74$ & 34074 & 9089 & 2 & Feb 1980 & SL7 & \\
\hline 34 Se-76 & 34076 & 9091 & 1 & Apr 1974 & SL17 & \\
\hline 34-Se-77 & 34077 & 9092 & 1 & Apr 1974 & SL31 & \\
\hline $34-5 e-78$ & 34078 & 9094 & 1 & Apr 1974 & SL12 & \\
\hline $34-\operatorname{se}-80$ & 34080 & 9097 & 1 & Apr 1974 & SL7 & \\
\hline $34-S e-82$ & 34082 & 9100 & 1 & Apr 1974 & SLA & \\
\hline $14-5 i$ & 14000 & 1314 & 3 & Feb 1974 & BF & \\
\hline 62-Sm-144 & 62144 & 9803 & 2 & Feb 1980 & & \\
\hline $62-5 m-147$ & 62147 & 9806 & 2 & Feb 1980 & SL59 & \\
\hline 62-Sm-148 & 62148 & 9807 & 1 & Feb 1980 & & \\
\hline $62-5 m-149$ & 62149 & 1319 & 1 & Nov 1978 & ML30,UN & \\
\hline 62-Sm-150 & 62150 & 9809 & 1 & Apr 1974 & SL12 & \\
\hline 62-Sm-151 & 62151 & 9810 & 1 & Feb 1980 & SL8 & \\
\hline $62-5 m-152$ & 62152 & 9811 & 1 & Feb 1980 & SL57 & \\
\hline 62-Sm-153 & 62153 & 9812 & 1 & Dec 1979 & & \\
\hline $62-5 m-154$ & 62154 & 9813 & 1 & Apr 1974 & SL18 & \\
\hline $50-5 n-112$ & 50112 & 9513 & 2 & Feb 1980 & SL12 & \\
\hline $50-5 n-114$ & 50114 & 9516 & 2 & Feb 1980 & SLS & \\
\hline $50-5 n-115$ & 50115 & 9517 & 1 & Apr 1974 & SL3 & \\
\hline $50-5 n-116$ & 50116 & 9518 & 1 & Apr 1974 & SL8 & \\
\hline $50-5 n-117$ & 50117 & 9519 & 1 & Apr 1974 & SL25 & \\
\hline $50-5 n-118$ & 50118 & 9521 & 1 & Apr 1974 & SL8 & \\
\hline $50-5 n-119$ & 50119 & 9522 & 1 & Apr 1974 & SL10 & \\
\hline $50-5 a-120$ & 50120 & 9524 & 1 & Apr 1974 & SLA8 & \\
\hline $50-5 n-122$ & 50122 & 9527 & 1 & Apr 1974 & SL6 & \\
\hline $50-5 n-123$ & 50123 & 9528 & 1 & Dec 1979 & & \\
\hline $50-5 n-124$ & 50124 & 9530 & 1 & Apr 1974 & SLS & \\
\hline $50-5 n-125$ & 50125 & 9531 & 1 & Dec 1979 & & \\
\hline $50-5 n-126$ & 50126 & 9533 & 1 & Dec 1979 & & \\
\hline $38-5 r-84$ & 38084 & 9179 & 2 & Feb 1980 & SL10 & \\
\hline $38-5 r-86$ & 38086 & 9182 & 1 & Apr 1974 & SL24 & \\
\hline $38-5 r-87$ & 38087 & 9183 & 1 & Apr 1974 & SL22 & \\
\hline $38-5 r-88$ & 38088 & 9185 & 1 & Apr 1974 & SL16 & \\
\hline $38-5 r-89$ & 38089 & 9186 & 1 & Dec 1979 & & \\
\hline $38-5 r-90$ & 38090 & 9187 & 1 & Dec 1979 & & \\
\hline $1-T-3$ & 1003 & 1169 & 2 & Feb 1965 & & \\
\hline 73-Ta-181 & 73181 & 1285 & 2 & $\operatorname{Jan} 1972$ & ML76,UN & \\
\hline 73-Ta-182 & 73182 & 1127 & 1 & Apr 1971 & ML10,UN & \\
\hline 65-Tb-159 & 65159 & 9857 & 1 & Feb 1980 & SL23 & \\
\hline $65-T b-160$ & 65160 & 9858 & 1 & Dec 1979 & & \\
\hline
\end{tabular}


Table 2. (continued)

\begin{tabular}{|c|c|c|c|c|c|c|}
\hline Nuelide & $\begin{array}{c}\text { LAW } \\
\text { identifier }\end{array}$ & $\begin{array}{l}\text { ENDF } \\
\text { MAT }\end{array}$ & MOD & $\begin{array}{l}\text { Eval. } \\
\text { date }\end{array}$ & $\begin{array}{l}\text { Resonance } \\
\text { data }\end{array}$ & $\begin{array}{c}\text { Thermal } \\
\text { MAT }\end{array}$ \\
\hline $\begin{array}{l}\text { 43-Tс-99 } \\
\text { 52-Te-120 }\end{array}$ & 43099 & 1308 & 2 & $\begin{array}{l}\text { Nov } 1978 \\
\text { Feb } 1980\end{array}$ & SL68,UN & \\
\hline $\begin{array}{l}52-T e-120 \\
52-T e-122\end{array}$ & $\begin{array}{l}52120 \\
52122\end{array}$ & $\begin{array}{l}9576 \\
9579\end{array}$ & $\begin{array}{l}2 \\
1\end{array}$ & $\begin{array}{l}\text { Feb } 1980 \\
\text { Apr } 1974\end{array}$ & & \\
\hline $\begin{array}{l}\text { 52-Te-122 } \\
\text { 52-Te-123 }\end{array}$ & $\begin{array}{l}52122 \\
52123\end{array}$ & $\begin{array}{l}9579 \\
9580\end{array}$ & $\begin{array}{l}1 \\
1\end{array}$ & & SL22 & \\
\hline $\begin{array}{l}52-1 \mathrm{C}-123 \\
52-T \mathrm{~T}-124\end{array}$ & $\begin{array}{l}52123 \\
52124\end{array}$ & $\begin{array}{l}9580 \\
9582\end{array}$ & $\begin{array}{l}1 \\
1\end{array}$ & $\begin{array}{l}\text { Apr } 1974 \\
\text { Apr } 1974\end{array}$ & SL26 & \\
\hline 52-Te-125 & $\begin{array}{l}52124 \\
52125\end{array}$ & $\begin{array}{l}9582 \\
9583\end{array}$ & $\begin{array}{l}1 \\
1\end{array}$ & $\begin{array}{l}\text { Apr } 1974 \\
\text { Apr } 1974\end{array}$ & SL36 & \\
\hline 52-Te-126 & $\begin{array}{l}52120 \\
52126\end{array}$ & 9585 & 1 & Apr 1974 & SL33 & \\
\hline 52-Te-127 & 52601 & 9587 & 1 & Dec 1979 & SL21 & \\
\hline 52-Te-128 & 52128 & 9588 & 1 & Apr 1974 & & \\
\hline 52-Te-129 & 52611 & 9590 & 1 & Dec 1979 & SL9 & \\
\hline 52-Te-130 & 52130 & 9591 & 1 & Apr 1974 & & \\
\hline 52-Te-132 & 52132 & 9594 & 1 & Dec 1979 & SL22 & \\
\hline 90-Th-230 & 90230 & 8030 & 1 & Nov 1977 & & \\
\hline $90-T h-232$ & 90232 & 1390 & 2 & Dec 1977 & $\begin{array}{l}\text { SL22 } \\
\text { ML435,UN }\end{array}$ & \\
\hline 22-Ti & 22000 & 1322 & 1 & Aug 1977 & ML435,UN & \\
\hline 92-U-232 & 92232 & 8232 & 1 & Nov 1977 & & \\
\hline 92-U-233 & 92233 & 1393 & 2 & Dec 1978 & & \\
\hline 92-U-234 & 92234 & 1394 & 3 & Jul 1978 & $\begin{array}{l}\text { AA83,UN } \\
\text { ML119,UN }\end{array}$ & \\
\hline 92-U-235 & 92235 & 1395 & 3 & Apr 1977 & & \\
\hline 92-U-236 & 92236 & 1396 & 3 & Jul 1978 & $\begin{array}{l}\text { SL130,UN } \\
\text { ML191,UN }\end{array}$ & \\
\hline 92-U-237 & 92237 & 8237 & 1 & Jul 1976 & $\begin{array}{l}\text { ML191,UN } \\
\text { SL27,UN }\end{array}$ & \\
\hline 92-U-238 & 92238 & 1398 & 3 & Jun 1977 & $\begin{array}{l}\text { SL27,UN } \\
\text { MLA44,UN }\end{array}$ & \\
\hline $23-V$ & 23000 & 1323 & 1 & Jan 1977 & & \\
\hline $74 W$ & 74000 & 1474 & 1 & Mar 1982 & ML197 & \\
\hline $74-W-182$ & 74182 & 1475 & 2 & Dec 1980 & $\begin{array}{l}\text { ML197 } \\
\text { ML69,UN }\end{array}$ & \\
\hline 74-W-183 & 74183 & 1476 & 2 & Dec 1980 & $\begin{array}{l}\text { ML69,UN } \\
\text { ML50,UN }\end{array}$ & \\
\hline $74 W-184$ & 74184 & 1477 & 2 & Dec 1980 & $\begin{array}{l}\text { ML50,UN } \\
\text { ML38,UN }\end{array}$ & \\
\hline $74-W-186$ & 74186 & 1478 & 2 & Dec 1980 & $\begin{array}{l}\text { ML38,UN } \\
\text { MLA0,UN }\end{array}$ & \\
\hline $54 \times x=-124$ & 54124 & 1335 & 1 & Mar 1978 & MLA & \\
\hline $54 \times e-126$ & 54126 & 1339 & 1 & Mar 1978 & MLA & \\
\hline $54-X e-128$ & 54128 & 1348 & 1 & Mar 1978 & ML10 & \\
\hline $54-X e-129$ & 54129 & 1349 & 1 & Mar 1978 & ML70 & \\
\hline $54-X e-130$ & 54130 & 1350 & 1 & Mar 1978 & ML12 & \\
\hline $54-X e-131$ & 54131 & 1351 & 1 & Mar 1978 & MLAO & \\
\hline $54-X e-132$ & 54132 & 1352 & 2 & Mar 1978 & MLA & \\
\hline $54-x e-133$ & 54133 & 9643 & 1 & Dec 1979 & & \\
\hline $54-X e-134$ & 54134 & 1354 & 1 & Mar 1978 & ML3 & \\
\hline $54-\mathrm{Xe}-135$ & 54135 & 1294 & 1 & Jun 1967 & & \\
\hline $54-x e-136$ & 54136 & 1356 & 1 & Mar 1978 & & \\
\hline $39-Y-89$ & 39089 & 9202 & 1 & Apr 1974 & SL16 & \\
\hline $39-Y-90$ & 39090 & 9204 & 1 & Dec 1979 & & \\
\hline $39 . Y-91$ & 39091 & 9206 & 1 & Dec 1979 & & \\
\hline & & & & & & \\
\hline
\end{tabular}


Table 2. (continued)

\begin{tabular}{|c|c|c|c|c|c|c|}
\hline Nuclide & $\begin{array}{c}\text { LAW } \\
\text { identifier }\end{array}$ & $\begin{array}{l}\text { ENDF } \\
\text { MAT }\end{array}$ & MOD & $\begin{array}{l}\text { Eval. } \\
\text { date }\end{array}$ & $\begin{array}{l}\text { Resonance } \\
\text { data }\end{array}$ & $\begin{array}{c}\text { Thermal } \\
\text { MAT }\end{array}$ \\
\hline 40-Zx & 40000 & 1340 & 2 & Apr 1976 & ML397 & \\
\hline $40-2 r .90$ & 40090 & 1385 & 2 & Apr 1976 & ML123 & \\
\hline 40-Zr-91 & 40091 & 1386 & 2 & Apr 1976 & ML94 & \\
\hline $40-z r-92$ & 40092 & 1387 & 2 & Apr 1976 & ML79 & \\
\hline $40-2 x-93$ & 40093 & 9232 & 1 & Dec 1979 & & \\
\hline 40-Zr-94 & 40094 & 1388 & 2 & Apr 1976 & ML71 & \\
\hline 40-Zr-95 & 40095 & 9234 & 1 & Dec 1979 & & \\
\hline $40-2 r-96$ & 40096 & 1389 & 2 & Apr 1976 & ML30 & \\
\hline 1/vet & 999 & & & & & \\
\hline Dowe factors & 900 & & & & & \\
\hline $\begin{array}{l}\text { Weighting } \\
\text { spectras }\end{array}$ & 99 & & & & & \\
\hline
\end{tabular}

See Sect. 2.4.

\section{RESONANCE SEILFSHEILDNG THE LAW LIBRARY}

A Nordheim Integral Treatment ${ }^{17}$ calculation is typically used for self-shielding resolved resonances with the LAW Library; the Bondarenko treatment ${ }^{18}$ is typically employed for unresolved resonance self-shielding. In the AMPX system, the ROLAIDS module provides a multizone, one-dimensional integral transport calculation for resolved resonance self-shielding. This module can be used with the LAW Library if the situation is such that the much simpler Nordheim Treatment is inadequate.

Nuclides that use single or multilevel Breit-Wigner data will have the information necessary for a Nordheim calculation placed on the AMPX master library. Those nuclides with unresolved data will also contain Bondarenko factor data.

The two nuclides, ${ }^{233} \mathrm{~J}$ and ${ }^{211} \mathrm{Pu}$, that used Adler-Adler data were processed as "infinite dilution" by AMPX to produce sets that require a ROLAIDS calculation to be made, if used in a situation that requires them to be resonance self-shielded. They were also processed using the NJOY $^{19}$ system to produce sets that contain Bondarenko data for all energy groups and can therefore be self-shielded in both the resolved and unresolved region using the Bondarenko method.

Four nonresonance nuclides were specially processed to take into account possible selfshielding effects arising from resonance structure in the point data. These were ${ }^{7} \mathrm{Li},{ }^{19} \mathrm{~F},{ }^{27} \mathrm{Al}$, and ${ }^{14} \mathrm{Si}$. These nuclides contain Bondarenko data for all energy groups.

Nuclides with unresolved data contain Bondarenko factors for self-shielding in this energy range, with a few exceptions. The nuclides W, Eu, Hf, and Mo all contain unresolved data. However, they are multi-isotope evaluations and do not have Bondarenko data on the master library because the unresolved resonance region varies from isotope to isotope. Our present Bondarenko treatment program (BONAMI) allows the factors to be given for an arbitrary energy range, but only one range can be specified in a cross-section set. The same is not true for the 
resolved range where the Nordheim calculation, as implemented in NITAWL, can treat variable ranges. The data for these sets in the LAW Library are, therefore, infinite dilution in the unresolved range with full self-shielding capabilities in the resolved range. Generally, this situation will not create a problem because sets of data are also provided for most isotopes that are part of the natural elemental form of these nuclides.

In the original version of the LAW-238 library, only s-wave resonance data were included in the resonance parameters that were passed to the Nordheim treatment in NITAWL. Subsequently, applications have been encountered, notably involving transport through iron and zirconium, whereby experimentally adding d-wave resonance data changed results by several percent. As a result, a special program was written to scan the ENDF/B-V library to locate all nuclides with d-wave and $p$-wave resonances, and to extract these data in a format which would allow them to be included in the 238-group library. A total of 21 nuclides was involved, as listed in Table 3. (Note that the self-shielding of these higher-order parameters is not automatic in NITAWL; the IQM parameter in the $1 S$ array is used to request their usage.)

\section{THERMALSCATTERING PROCESSING}

As mentioned earlier, several of the sets of data required the use of the ENDF/B scattering law data files. For all other nuclides, $P_{3}$-scattering matrices were generated based on free-gas scattering. For all nuclides except those noted below, thermal-scattering matrices were generated at 300,500 , and $900 \mathrm{~K}$.

For water-bound hydrogen (ID $=1001$ ) and $\mathrm{D}_{2} \mathrm{O}$-bound deuterium (ID $\left.=1002\right)$, thermal matrices are given at $296,350,400,450,500,500,600,800$, and $1000 \mathrm{~K}$. $350 \mathrm{~K}$

For polyethylene-bound hydrogen (ID $=1901$ ), thermal matrices are given at 296 and

For beryllium in metallic form (ID $=4309$ ), thermal matrices are given at $296,400,500$, $600,700,800,1000$, and $1200 \mathrm{~K}$.

For carbon in graphite (ID $=6000$ ), thermal matrices are given at $296,400,500,600$, $700,800,1000,1200,1600$, and $2000 \mathrm{~K}$.

\section{SPECIAL WEIGHTING FUNCTIONS PROVIDED TO COLLAPSE 238-GROUP LIBRARY}

A special material is included in the LAW Library with an identifier of 99 , which contains 238-group weighting spectra for a variety of situations one might find of interest.

As mentioned in Sect. 1.3, it is easy to tell the AMPX MALOCS module to collapse the cross-section sets in a library over the spectrum that was used to obtain the 238-group library. An option is also provided, wherein one can select a function from any set of data on the library and use this spectrum to collapse all nuclides on the library. This latter option is useful for producing an "application-specific" collapsed library. 
Table 3. ENDFB-V nuclides with p-and d-wave reconance parameters

\begin{tabular}{|c|c|c|c|c|c|c|c|c|}
\hline Nuctide & Identifier & MAT & Isotope & Abundance & s-wave & p-wave & d-wave & $\begin{array}{c}\text { Energy } \\
\text { range }\end{array}$ \\
\hline $\begin{array}{l}\text { Co.s9 } \\
\mathrm{C}\end{array}$ & $\begin{array}{l}27059 \\
24000\end{array}$ & $\begin{array}{l}1327 \\
1324\end{array}$ & $\begin{array}{l}59 \\
50 \\
52 \\
53 \\
54\end{array}$ & $\begin{array}{l}1.0 \\
0.043 \\
0.838 \\
0.095 \\
0.024\end{array}$ & $\begin{array}{l}72 \\
38 \\
14 \\
15 \\
10\end{array}$ & $\begin{array}{r}107 \\
20 \\
59 \\
10 \\
14\end{array}$ & & $\begin{array}{l}1 \times 10^{-5}-8.5 \times 10^{4} \\
1 \times 10^{-5}-6.93 \times 10^{5}\end{array}$ \\
\hline $\mathbf{F e}$ & 26000 & 1326 & $\begin{array}{l}54 \\
56 \\
57 \\
58\end{array}$ & $\begin{array}{l}0.058 \\
0.917 \\
0.022 \\
0.003\end{array}$ & $\begin{array}{r}16 \\
15 \\
21 \\
5\end{array}$ & $\begin{array}{l}58 \\
69 \\
29\end{array}$ & $\begin{array}{l}56 \\
43\end{array}$ & $155-4 \times 10^{5}$ \\
\hline \multirow[t]{6}{*}{$\begin{array}{l}\text { Mn-55 } \\
\text { Mo }\end{array}$} & $\begin{array}{l}25055 \\
42000\end{array}$ & $\begin{array}{l}1325 \\
1321\end{array}$ & $\begin{array}{l}55 \\
92 \\
94\end{array}$ & $\begin{array}{l}1.0 \\
0.158 \\
0.090\end{array}$ & $\begin{array}{r}43 \\
1 \\
1\end{array}$ & 96 & & $\begin{array}{l}1.0 \times 10^{-5}-1.3 \times 10^{5} \\
4-1000\end{array}$ \\
\hline & & & 95 & 0.157 & 7 & 11 & & \\
\hline & & & 96 & 0.165 & 1 & 1 & & \\
\hline & & & 97 & 0.095 & 10 & 7 & & \\
\hline & & & 98 & 0.238 & 4 & 1 & & \\
\hline & & & 100 & 0.096 & 1 & 1 & & \\
\hline $\begin{array}{l}\mathrm{Na}-23 \\
\mathrm{Nb}-93 \\
\mathrm{Ni}\end{array}$ & $\begin{array}{l}11023 \\
41093 \\
28000\end{array}$ & $\begin{array}{l}1311 \\
1189 \\
1328\end{array}$ & $\begin{array}{l}23 \\
93 \\
58\end{array}$ & $\begin{array}{l}1.0 \\
1.0 \\
0.681\end{array}$ & $\begin{array}{l}4 \\
95 \\
30\end{array}$ & $\begin{array}{r}12 \\
123 \\
36\end{array}$ & 7 & $\begin{array}{l}600-5 \times 10^{5} \\
1 \times 10^{-5}-7500 \\
1 \times 10^{-5}-6.9 \times 10^{5}\end{array}$ \\
\hline & & & $\begin{array}{l}60 \\
62 \\
64\end{array}$ & $\begin{array}{l}0.265 \\
0.040 \\
0.015\end{array}$ & $\begin{array}{l}40 \\
35 \\
24\end{array}$ & $\begin{array}{l}49 \\
35 \\
44\end{array}$ & & \\
\hline $\mathbf{R b}-\mathbf{8 5}$ & 37085 & 1360 & 85 & 1.0 & 75 & 12 & & $1 \times 10^{-5}-2 \times 10^{4}$ \\
\hline $\mathbf{R b}-87$ & 37087 & 1341 & 87 & 1.0 & 14 & 1 & & $1 \times 10^{-5}-3 \times 10^{4}$ \\
\hline $\mathbf{R} \mathbf{h}-103$ & 45103 & 1310 & 103 & 1.0 & 59 & 60 & & $1 \times 10^{-5}-1500$ \\
\hline & 32000 & 1347 & 32 & 0.950 & 5 & 92 & 16 & $1 \times 10^{-5}-1.1 \times 10^{6}$ \\
\hline & & & $\begin{array}{l}33 \\
34\end{array}$ & $\begin{array}{l}0.008 \\
0.042\end{array}$ & $\begin{array}{l}6 \\
4\end{array}$ & $\begin{array}{r}33 \\
9\end{array}$ & & \\
\hline Tc-99 & 43099 & 1308 & 99 & 1.0 & 42 & 25 & & $1 \times 10^{-5}-800$ \\
\hline Th-232 & 90232 & 1390 & 232 & 1.0 & 241 & 192 & & $5-4000$ \\
\hline U-238 & 92238 & 1398 & 238 & 1.0 & 116 & 280 & & $1-4000$ \\
\hline $\mathbf{Z x}$ & 40000 & 1340 & 90 & 0.515 & 34 & 84 & 5 & $1 \times 10^{-5}-9 \times 10^{4}$ \\
\hline & & & 91 & 0.112 & 35 & 58 & & $1 \times 10^{-5}-2.45 \times 10^{4}$ \\
\hline & & & 92 & 0.171 & 15 & 63 & & $1 \times 10^{-5}-9 \times 10^{4}$ \\
\hline & & & $\begin{array}{l}94 \\
96\end{array}$ & $\begin{array}{l}0.174 \\
0.028\end{array}$ & $\begin{array}{r}22 \\
8\end{array}$ & $\begin{array}{l}48 \\
21\end{array}$ & & $\begin{array}{l}1 \times 10^{-5}-9 \times 10^{4} \\
1 \times 10^{-5}-9 \times 10^{4}\end{array}$ \\
\hline$Z_{\mathrm{r}-90}$ & 40090 & 1385 & 90 & 1.0 & 34 & 84 & 5 & $1 \times 10^{-5}-9 \times 10^{4}$ \\
\hline $\mathrm{Z}_{\mathrm{r}-91}$ & 40091 & 1386 & 91 & 1.0 & 35 & 58 & & $1 \times 10^{-5}-2.45 \times 10^{4}$ \\
\hline $\mathrm{Z} \times-92$ & 40092 & 1387 & 92 & 1.0 & 15 & 63 & & $1 \times 10^{-5}-9 \times 10^{4}$ \\
\hline Zr-94 & 40094 & 1388 & 94 & 1.0 & 22 & 48 & & $1 \times 10^{-5}-9 \times 10^{4}$ \\
\hline Zr-96 & 40096 & 1389 & 96 & 1.0 & 8 & 21 & & $1 \times 10^{-5}-9 \times 10^{4}$ \\
\hline
\end{tabular}


Several spectra are included, as listed below:

1. spectrum based on a fuel cell from a $17 \times 17$ Westinghouse light-water-reactor (LWR) assembly and identified by 9001 ,

2. spectrum designed for use with the Molten Salt Reactor Experiment (MSRE) fuel storage tanks at ORNL and identified by 9002 ,

3. average spectrum in a $27-\mathrm{cm}$ carbon steel for use in cask shielding studies and identified by 9003 ,

4,5. average spectra in an $18.6-\mathrm{cm}$ lead/13-cm resin shield for use in shielding cask studies (the flux in the lead region is identified by 9004 and that in the resin region by 9005), and

6. average spectrum in a $50-\mathrm{cm}$ concrete shield for cask shielding studies and identified by 9006.

In the latter four cases, the spent fuel source is a Westinghouse $15 \times 15$ as sombly with initial enrichment of $3.0 \mathrm{wt} \%$ and burned to 30 gigawatt days per metric ton of uranium ( $30 \mathrm{GWd} / \mathrm{MTU}$ ) and cooled for 5 years. 


\section{LIBRARY PRODUCTION PROCEDURES}

A sequence of three programs was rus for each set of data on the LAW Library. The XLACS program reads ENDF/B data and produces a set of multigroup cross sections in AMPX library format. The RADE program was used to perform quality and consistency checks on the multigroup data. The VASELINE program was used to make plots of the multigroup and point data, as a further aid to testing.

In the case of nuclides with unresolved resonance data, this sequence of three programs was augmented by three more programs. PRUDE processes unresolved data into a point format, TABU processes these point data into Bondarenko factors, and, finally, the UNITAB program couples the Bondarenko factors with the master library produced by XLACS.

Figure 2 is a flow chart of the above procedure.

As mentioned earlier, ${ }^{233} \mathrm{U}$ and ${ }^{241} \mathrm{Pu}$ were processed by NJOY. In these cases, the sequence of NJOY modules-RECONR, BROADR, UNRESR, THERMR, GROUPR, and AMPXR - was run. RECONR is used to produce a "point" ENDF file with data at $0 \mathrm{~K}$ from the basic ENDF file. BROADR takes this file and Doppler-broadens the data to 300,500, 900 and $2100 \mathrm{~K}$. UNRESR processes the unresolved ENDF parameters and produces self-shielded point files at the temperatures mentioned above and for background cross-section values of 1 , $10,100,1000,10^{4}, 10^{5}$, and $10^{10}$ barns. THERMR produced thermal-scattering matrices based on the free-gas model at $300,500,900$, and $2100 \mathrm{~K}$. GROUPR reduces the point data to groupaveraged form, and, finally, AMPXR converts from the GENDF format written by GROUPR to the AMPX master format. This procedure is shown schematically in Fig. 3.

After all nuclides were produced, the AJAX program was used to collect all sets of data into a single AMPX master library. This library was subsequently run through the COMET program that forces consistency between group-averaged scattering cross sections and scattering transfer arrays. Subsequently, it writes a new master library.

The final operation for the library is one required for the thermal-scattering matrices. The familiar free-gas treatment assumes a constant value of the free-atom cross section at energies well above thermal. The equations that arise combine this constant cross section with free-gas-scattering kinematies and Doppler broadening. Unfortunately, many nuclides have resonances that are located in or near the thermal region. Even nuclides that have flat scattering cross sections have their cross-section values affected by resonances away from the thermal range.

Since thermal scattering is not that important for most heavier nuclides (which are the nuclides with low-energy resonances), the following simple scheme was selected. The thermalscattering matrices are generated by using a free-atom cross section derived from using the scattering radius given as part of the resonance data on an ENDF/B evaluation. These matrices are then normalized to the group-averaged values determined by Doppler broadening and weighting point ENDF/B data. This method is a crude way of producing matrices. However, it includes the effects of thermal resonance structure, Doppler broadening, and thermalscattering kinematics. This last normalization is performed by the FRESH program. The library it produces is the 238-group LAW Library. 


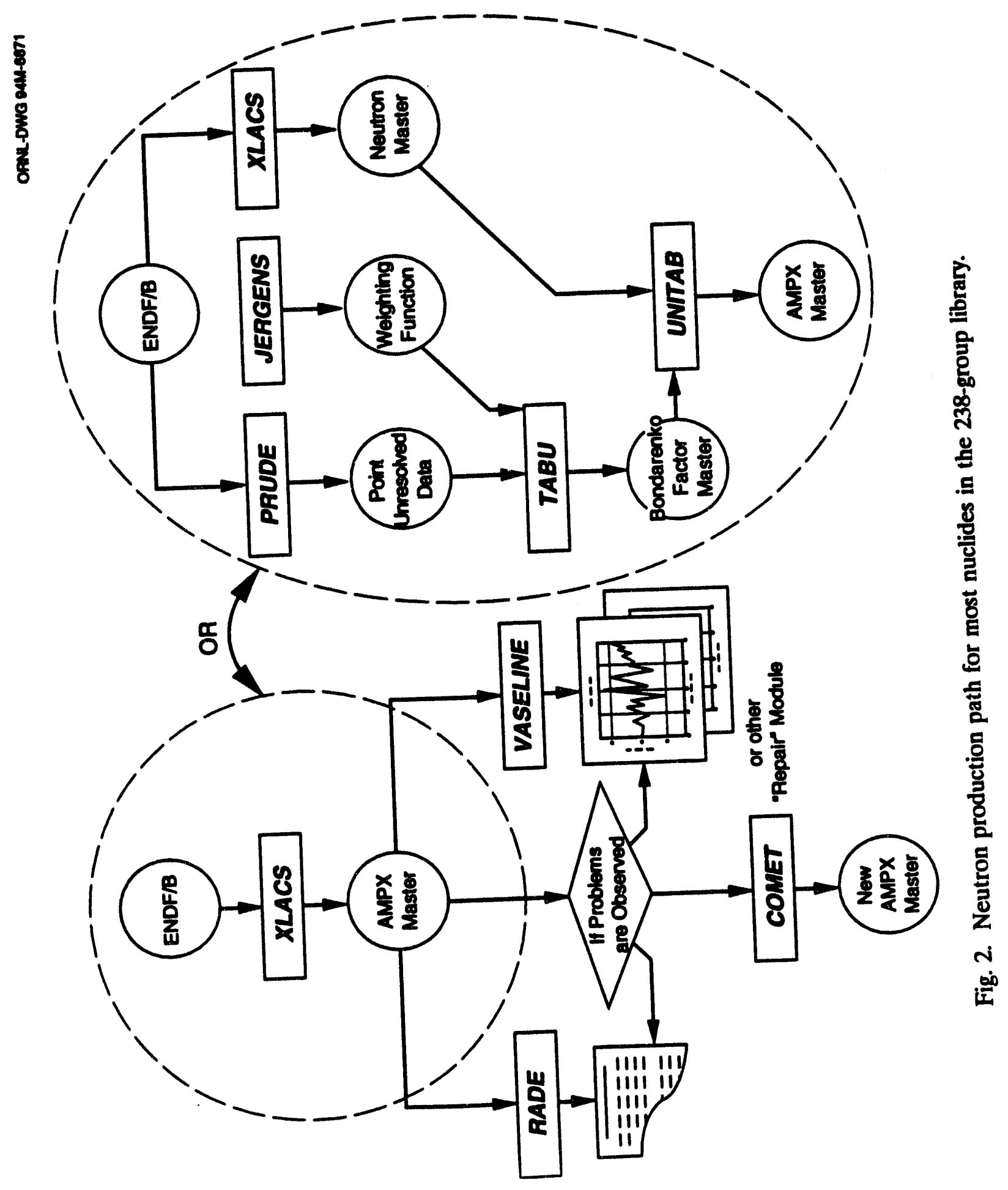




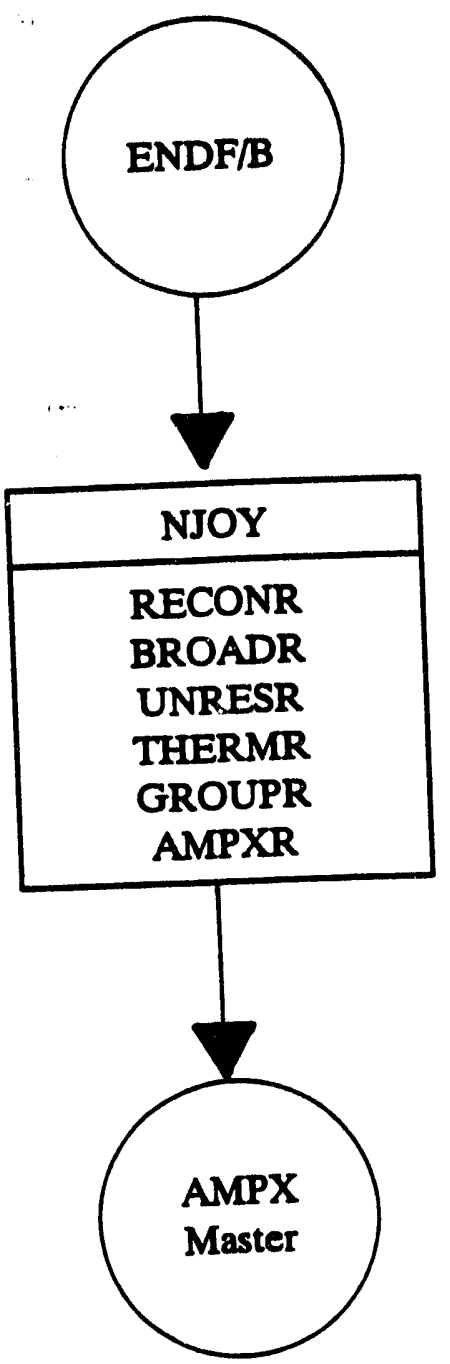

Fig. 3. Processing procedure for ${ }^{233} \mathrm{U}_{92}$ and ${ }^{241} \mathrm{Pu}_{94}$. 


\section{IIBRARY TESTING AND CONCLUSIONS}

\subsection{HISTORY OF ENDF/B-V DATA TESTMNG}

The ENDF/6-V Cioss-Section Library ${ }^{2}$ was released in 1980, and the ENDF/B-V Benchmark Data Testing Report ${ }^{20}$ was published in August 1982. The ${ }^{299}$ Pu evaluation was revised in June 1983 (ENDF/B-V Revision 2). Additional ENDF/B-V data testing was done to asseas the impact of the revised ${ }^{20} \mathrm{Pu}$ evaluation.

\section{DATA TESTING OF THE LAW-238 LIBRARY}

The objective of the present data testing effort is to verify the new cross-section processing procedures used in generating the LAW-238 multigroup cross sections. The LAW-238 testing to date includes calculations for $7 \mathrm{Pu}$-fueled, $1{ }^{223} \mathrm{U}$-fueled, and $25{ }^{235} \mathrm{U}$-fueled benchmarks. There are 15 fast and 18 thermal benchmarks. Of these 33 benchmarks, 28 can be considered to be CSEWG benchmarks. We were able to demonstrate that, for most benchmarks, the calculations using the LAW-238 cross-section library give results that are very close to those obtained with other ENDF/B-V-based libraries. The results discussed in Sects. 4.3-4.4 were performed after initial preparation of the LAW library. Oxygen data from ENDF/B-V were used in this initial testing except as noted in Sect. 4.4.7 The analyses were repeated after selected resonance nuclides were modified to add $p$-wave and $d$-wave resonance data and selected nonresonance nuclides were modified to include shielding factors for highenergy resonance-like structure. No significant changes in the results or the observations noted below were seen in the final analyses. Results from the final test analyses are shown in Appendix $A$ and compared to the initial test results discussed below.

\section{FAST-REACTOR BENCHMARKS}

Specifications for the CSEWG fast reactor benchmarks are given in ref. 21 and will not be given in this document. Calculations have been done for ten of the CSEWG fast benchmarks. The values of $k_{\text {eff }}$ using the LAW-238 Library are shown in Table 4 and are compared with calculated values using the 174-group VITAMIN-E Library ${ }^{22}$ and with results from Bob MacFarlane ${ }^{23}$ of Los Alamos National Laboratory. For eight of the ten benchmarks in Table 4, the calculated $k_{\text {eff }}$ values are in good agreement with the other two ENDF/B-V calculations. The LAW-238 calculated value for the JEZEBEL benchmark is a little lower than the corresponding values from the other calculations. By calculating a sphere of pure ${ }^{209} \mathrm{Pu}$ with a radius of $4.967 \mathrm{~cm}$ and ${ }^{209} \mathrm{Pu}$ density of $19.70 \mathrm{gm} / \mathrm{cc}$, we were able to show that the JEZEBEL LAW-238/VITAMIN-E difference of $0.4 \%$ is due to differences in the ${ }^{239} \mathrm{Pu}$ cross sections in the two libraries since the numerical benchmark (pure ${ }^{239} \mathrm{Pu}$ ) is also different by the same amount. The calculated $k_{\text {eff }}$ for ZPR-6/6A using the LAW-238 Library is $0.31 \%$ higher than both the VITAMIN-E and LANL calculations. 
Table 4. Fast-reactor benchmarks

\begin{tabular}{|c|c|c|c|}
\hline Benchmark & $\begin{array}{l}\text { VITAMIN-E } \\
\text { 174-GROUP }\end{array}$ & $\begin{array}{l}\text { LANL } \\
\text { 80-GROUP }\end{array}$ & LAW-238 \\
\hline JEZEBEL" & 0.9983 & 0.9961 & 0.9944 \\
\hline JEZEBELPU & 1.0021 & 0.9999 & 0.9986 \\
\hline JEZEBEL-23 ( $\left.{ }^{238} U\right)$ & 0.9935 & 0.9940 & 0.9959 \\
\hline GODIVA $^{b}$ & 0.9966 & 0.9973 & 0.9963 \\
\hline FLATTOP-25 & 1.0047 & 1.0065 & 1.0046 \\
\hline BIG TEN ${ }^{c}$ & 1.0103 & 1.0121 & 1.0103 \\
\hline ZPR-3/11 & 1.0126 & & 1.0119 \\
\hline ZPR-3/12 & 1.0047 & & 1.0068 \\
\hline ZPR-6/6A & 0.9921 & 0.9922 & 0.9952 \\
\hline ZPR-6/7 & 1.0037 & 0.9975 & 1.0022 \\
\hline
\end{tabular}

is $S_{16}$ to $S_{\infty}$ correction: -0.0021 was applied.

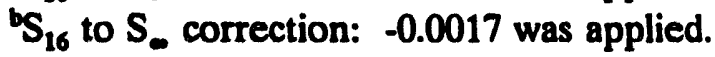

LANL calculations for BIG TEN used 175 groups.

\subsection{THERMAL REACTOR BENCHMARKS}

Data testing of ENDF/B.V for thermal reactors is reported in ENDF-311. ${ }^{20}$ In the present work, a subset of the cases considered in ref. 20 will be considered. The main emphasis will be for ${ }^{235} \mathrm{U}$-fueled benchmarks, with only limited coverage of Pu-fueled assemblies.

\subsubsection{ORNL Spheres (uranyl nitrate in water)}

Benchmark specifications for the ORNL spheres are available in ENDF-202.21 A detailed analysis of these systems is given in ref. 24. This series of benchmarks consists of five unreflected spheres of ${ }^{235} U$ (as uranyl nitrate) in water, three of them poisoned with boron. The ${ }^{235} \mathrm{U}$ enrichment is approximately $93 \%$. Values of $\mathrm{k}_{\text {eff }}$ calculated by XSDRNPM for $\mathrm{S}_{8}, \mathrm{P}_{3}$ using the LAW-238 Library are given in Table 5. Calculations of the ORNL spheres were also done with the ANSL-V 39- and 99-group libraries ${ }^{25}$ and for ORNL-1, -2 , and -10 by Bob MacFarlane 26 using 187 groups. The other results are compared with the LAW-238 values in Table 5 . We note 
Table 5. ORNL spheres (uranyl nitrate in water)

\begin{tabular}{lllll}
\hline & ANSLV & ANSLV & LANL & \\
BENCHMARK & 39-GROUP & 99-GROUP & 187-GROUP & LAW-238 \\
\hline ORNL-1 & 1.0025 & 1.0012 & 1.0008 & 1.0007 \\
ORNL-2 & 1.0023 & & 1.0005 & 1.0005 \\
ORNL-3 & 0.9993 & & & 0.9975 \\
ORNL-4 & 1.0007 & & & 0.9989 \\
ORNL-10 & 1.0012 & 1.0002 & 0.9980 & 0.9993 \\
\hline
\end{tabular}

a trend for the calculated $k_{\text {eff }}$ of a given benchmark to decrease by about $0.2 \%$ as the number of groups is increased (from 39 to 187 or 238). Also, the LANL-187 and LAW-238 values are in excellent agreement for ORNL-1 and ORNL-2. For some reason, the agreement for ORNL-10 is not quite as good.

\subsection{Uranium Lattice Benchmark Teating}

Benchmark specifications for TRX-1 and TRX-2 and the BAPL-1, -2 , and -3 lattices are given in ENDF-202.21 The benchmarks are $\mathrm{H}_{2} \mathrm{O}$-moderated lattices of slightly enriched (1.3 wt \%) uranium rods in a triangular pattern. The TRX rods are uranium metal, and the BAPL rods are uranium oxide. The clad is aluminum for all of these lattices. The lattices directly test the ${ }^{235} U$ fission- resonance integral and thermal-fission cross section. They also test ${ }^{238} U$ shielded resonance capture and the thermal-capture cross section. They are sensitive to the ${ }^{238} \mathrm{U}$ fastfission cross section, ${ }^{25} \mathrm{U}$ inelastic scattering, and the ${ }^{235} \mathrm{U}$ fission spectrum. The scattering and thermal absorption cross sections of $\mathrm{H}_{2} \mathrm{O}$ are also important. To account for leakage, an homogenized B-3 calculation with an input "height" corresponding to the total buckling of each lattice was done. For the BAPL lattices, a two-region correction was added to the calculated $\mathbf{k}_{\text {eff }}$ values. For TRX-1 and TRX-2, the two-region corrections are small and were assumed to be zero.

Values of $k_{\text {eff }}$ calculated by XSDRNPM using the ANSL-V (99-group) and the LAW-238 libraries are given in Table 6. Calculations of TRX-1 and BAPL-1 were also done with the "NMG" 99-group library (same specifications as for LAW-238 but in the ANSL-V 99-group structure). The LAW-238 and ANSL-V results agree to within $0.15 \%$ for all five lattices shown in Table 6. We note that for TRX-1, the LAW-238 and "NMG-99" values are exactly the same while for BAPL-1 the NMG-99 $\mathrm{k}_{\mathrm{eff}}$ is higher by +0.0018 . Thus we appear to have a group structure effect for BAPL-1 but not for TRX-1. The TRX and BAPL lattices were calculated by Don Craig ${ }^{27}$ in 1982 and are documented in AECL-7690. Craig's results are also included in Table 6. Craig's calculations were made using the integral transport code RAHAB ${ }^{28}$ with the 
Table 6. Uranium- $\mathrm{H}_{2} \mathrm{O}$-moderated lattices

\begin{tabular}{lllll}
\hline Benchmark & $\begin{array}{c}\text { ANSL-V } \\
\text { 99-GROUP }\end{array}$ & NMG-99 & CRAIG & LAW-238 \\
\hline TRX-1 & 0.9901 & 0.9915 & 0.9951 & 0.9920 \\
TRX-2 & 0.9970 & & 0.9971 & 0.9962 \\
BAPL-1 & 0.9986 & 0.9999 & 1.0011 & 0.9986 \\
BAPL-2 & 1.0001 & & 1.0015 & 1.0002 \\
BAPL-3 & 1.0024 & & 1.0022 & 1.0024 \\
\hline
\end{tabular}

Data taken from ref. 9.

resonance reaction rates being obtained from the OZMA ${ }^{29}$ program for the most important nuclides (i.e., ${ }^{235} U$ and ${ }^{239} U$ for these lattices). OZMA solves the neutron transport equation for a unit cell; the discrete-ordinates option was used in Craig's work. OZMA is similar to ROLAIDS in that ${ }^{235} U{ }^{233} U$ resonance overlap effects are accounted for. In this respect, the OZMA calculation is more accurate than NITAWL, which only treats one resonance nuclide at a time. Eighty-nine energy groups were used for RAHAB, with $\mathbf{4 2}$ groups in the thermal range below $4 \mathrm{eV}$; neutron upscattering was included up to $4 \mathrm{eV}$. These choices appear to be entirely adequate for these calculations. Craig's calculated $k_{\text {eff }}$ for TRX-1 is $0.31 \%$ higher than the LAW-238 value, and his BAPL-1 calculated value is $0.25 \%$ higher. The other lattices agree to within $0.13 \%$ or better. The LAW-238 values for the BAPL lattices have a significant trend with $\mathrm{H}_{2} \mathrm{O} /$ fuel ratio, while Craig's results show a much smaller variation.

A trend for the U-metal lattices to be about $0.3 \%$ lower than the uranium-oxide lattices is also observed in Table 6. The combined methods and differential data estimated uncertainty for the TRX-2 lattice was $0.5 \%$ in ref. 20 . The impact of uncertainties in ${ }^{20} U$ resonance parameters (i.e., the ${ }^{258} U$ shielded capture) on the performance parameters of the TRX-1 and BAPL-1 lattices was investigated in a 1983 paper..$^{30}$ The ${ }^{238} \mathrm{U}$ resonance parameter sensitivities for the TRX-1 and BAPL-1 lattices were found to be almost identical. Thus, the TRX-1/BAPL$1 \Delta k$ of about $0.3 \%$ cannot be eliminated by changing the ${ }^{288} U$ resonance parameters, and some other explanation must be sought. We should consider the possibility that the resonance shielding method used in NITAWL may be contributing to the bias between the TRX and BAPL lattices. 


\subsection{Uranyl Fluoride $\left(\mathrm{UO}_{2} \mathrm{~F}_{2}\right) / \mathrm{H}_{2} \mathrm{O}$ Moderator}

The L-series, Table 7, meet the requirements for benchmarks and are recognized as good candidates for data testing by the Thermal Reactor Data Testing and Applications Subcommittee of CSEWG. The L-eeries benchmarks are documented in EPRI NP-5058. ${ }^{31}$ The LAW-238 calculated $k_{\text {exf }}$ values in Table 7 are in good agreement with the LANL 187-group values. ${ }^{26}$ The calculated leakage for these benchmarks ${ }^{31}$ varies from 0.1742 for L-9 to 0.4687 for L-7. The calculations with the LAW-238 cross sections show a trend with leakage. This trend was not seen in the calculations by Schmidt and Rose (see Table 2-2 of ref. 31) using the SAM-CE Monte Carlo program, but has been seen by other data testers (e.g., the LANL-187 values in Table 8). A plot of the LAW-238 calculated $k_{\text {eff }}$ vs leakage is given in Fig. 4. The slope of the line in Fig. 4 is $\mathbf{0 . 0 1 1 5}$, which shows the trend of $k_{\text {eff }}$ to increase with leakage. This is excellent agreement with the LANL-187 results (compare the calculated $k_{\text {eff }}$ values for the L-9 and L-7 cases) which show a slope of 0.0157 . The trend with leakage is also seen by other CSEWG data testers and is generally accepted by the Thermal Reactor Data Testing and Applications Subcommittee. The impact of the ENDF/B-VI ${ }^{16} \mathrm{O}$ evaluation will be to lower the calculated $k_{m}$ values of the L-series benchmarks and also to lower the slope of the line in Fig. 4 . The L-eeries benchmark calculations were repeated using the ENDF/B-VI oxygen cross sections and are discussed in Sect. 4.4.7.

Table 7. Uranyl fluoride $\left(\mathrm{UO}_{2} \mathrm{~F}_{2}\right) / \mathrm{H}_{2} \mathrm{O}$ moderator

\begin{tabular}{ccc}
\hline Benchmark & LANL-187 & LAW-238 \\
\hline L-7 & 1.0099 & 1.0081 \\
L-8 & 1.0090 & 1.0088 \\
L-9 & 1.0053 & 1.0052 \\
L-10 & & 1.0090 \\
L-11 & & 1.0036 \\
\hline
\end{tabular}

Table 8. Homogeneous $\mathrm{Pu}-\mathrm{H}_{2} \mathrm{O}$ assemblies

\begin{tabular}{lcl}
\hline Benchmark & LANL & LAW-238 \\
\hline PNL-3 & $1.0003 \pm 0.0023$ & 0.9982 \\
PNL-6B & $1.0118 \pm 0.0024$ & 1.0102 \\
PNL-11 & $1.0098 \pm 0.0022$ & $1.0023 \pm 0.0034$ \\
\hline
\end{tabular}

Data taken from ref. 23. 


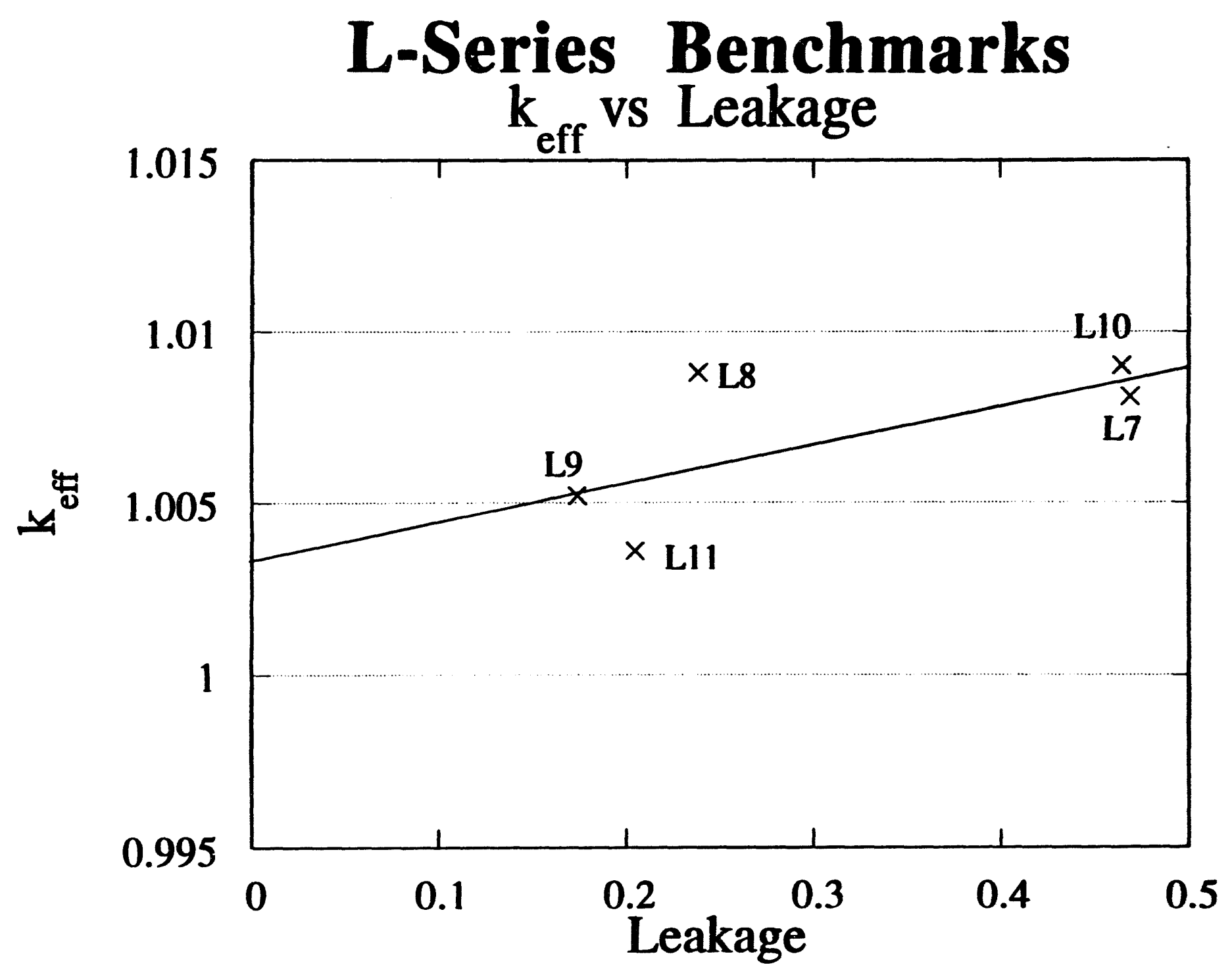

Fig. 4. Calculated $k_{\text {eff }}$ vo leakage. 


\subsubsection{Homogeneous Pu-B2O Anemblien}

As noted previously, the main emphasis in this work is for ${ }^{25} \mathrm{U}$ benchmarls with only limited coverage of Pu-fueled aseemblies. Three homogeneous $\mathrm{Pu}-\mathrm{H}_{2} \mathrm{O}$ assemblies have been calculated using the LAW-238 Library. KENO V.a was used for the PNL-11 calculation. The calculated $k_{\text {uf }}$ values are compared in Table 8 with values from LANL (see ref. 20). When the Monte Carlo uncertainties are taken into account, the level of agreement between the LAW-238 and LANL values is fairty good. Based on the limited amount of testing done thus far, results obtained for these bomogeneous $\mathrm{Pu}-\mathrm{H}_{2} \mathrm{O}$ asemblies are in good agreement with those obtained by other ENDF/B-V data testers.

\subsubsection{Other Fest Benchmarts}

Five additional uranium benchmarks have also been analyzed. Even though they are not CSEWG benchmarks, they are of interest for ENDF/B-V data testing. The H2OX-1 benchmark was analyzed by Slater. ${ }^{2 S}$ Systems similar to H2OX-1 are of considerable interest to the criticality safety community (e.g., accident analysis of the ANS reactor). The two uranium hydride cases, UH3-UR and UH3-NI, differ significantly from the fast benchmarks considered in Table 4. The HISS(HUG) and HISS(HPG) benchmarks are sensitive to ${ }^{235} \mathrm{U}$ fission and ${ }^{239} \mathrm{Pu}$ fission in the resolved resonance range and as such are unique in this respect and thus represent a valuable addition to the benchmarks considered.

Calculated $k_{\text {eff }}$ values for these five additional benchmarks are given in Table 9. The H2OX-1 benchmark is a light-water-reflected U-metal sphere; ${ }^{25,32}$ the calculated value of $k_{\text {eff }}$ from ref. 25 is given in Table 9. We note that the ANSL-V 39-group calculated $k_{\text {ems }} 1.0029$, is slightly better (i.e., closer to 1) than the LAW-238 value. The impact of the ENDF/B-VI oxygen cross sections will be to lower the H2OX-1 calculated $k_{\text {eff }}$ values slightly (see Appendix $A$ ).

Table 9. Other fast benchmarks

\begin{tabular}{lllc}
\hline & $\begin{array}{c}\text { VITAMIN-E } \\
\text { 174 GRP }\end{array}$ & $\begin{array}{l}\text { ANSLV } \\
\text { 39 GRP }\end{array}$ & LAW-238 \\
\hline H2OX-1 & & 1.0029 & 1.0047 \\
UH3-UR & 1.0065 & & 1.0098 \\
UH3-NI & 1.0137 & & 1.0245 \\
HISS(HUG) & 1.0258 & & 1.0241 \\
HISS(HPG) & 0.9949 & 1.0005 \\
\hline
\end{tabular}


The UH3-UR and UH3-NI benchmarks are enriched-uranium-hydride critical asemblies with natural uranium and nickel reflectors, respectively. These critical experiments were done on the Topay machine. ${ }^{33}$ In the initial calculation of the UH3-NI benchmark we determined that the Ni cross sections were being treated as infinitely dilute. After investigation we found that CSASN had identified $\mathrm{Ni}$ as a nonresonance material. We also found that both $\mathrm{Ni}$ and $\mathrm{Cr}$ were treated as nonreconance materials in some of the fast benchmarks in Table 4. Some other materials were also incorrectly flagged as nonresonance materials. CSASN was revised to correctly set the resonance flags for a number of materials, and the ZPR-3/11, ZPR-3/12, ZPR-6/6A, and ZPR-6/7 cases were reescecuted. Results given in Table 4 are from the corrected cases. The UH3-NI case has been executed using ID 28301, which is weighted using $1 /\left(\mathrm{E}_{\sigma_{T}}\right)$ weighting. This adjusted value will be closer to the correct weighting for $\mathrm{Ni}$ in the resonance range. However, in the fission spectrum range the weighting should be more like a fission spectrum divided by $\sigma_{\mathrm{T}}$. The value of $k_{\text {ex }}$ given in Table 9 for the UH3-NI benchmark was obtained using ID 28301 [i.e., for $1 /\left(E_{\sigma_{T}}\right)$ weighting]. The calculated $k_{\text {eff }}$ for the UH3-NI. benchmark using VITAMMN-E is 1.0137, which gives a $\Delta k$ of 0.0109 compared with the LAW-238 value. Another result of interest for the UH3-NI benchmark is that the leakage fraction using VITAMIN-E cross sections is $33.18 \%$, compared with only $30.02 \%$ leakage with the LAW-238 Library. The VITAMIN-E/LAW-238 $\Delta k$ can be explained primarily as a leakage effect that is due to "group structure" differences. This is illustrated in Figs. 5 and 6, which show the Ni total cross sections from the LAW-238 and VITAMIN-E libraries, respectively.

The HISS benchmarks are documented in ref. 34. HISS(HUG) has homogeneous uranium-graphite fuel; the HISS(HPG) benchmark has homogeneous plutonium-graphite fuel. The FISS cases are modeled as an infinite homogeneous medium. In other words, the calculation is $k$. rather than $k_{\text {err }}$. The HISS cases have also been executed using VITAMIN-E cross sections. We note that the HISS(HUG) benchmark calculated $k_{\text {. }}$ is about 1.0250 with both LAW-238 and VITAMIN-E cross sections, which can be compared with the "experimental" value of $1.0000 \pm 0.0040 .{ }^{34}$ The HISS(HPG)-calculated $k_{\text {c }}$ using the LAW-238 cross sections is 1.0005; the corresponding VITAMIN-E value is 0.9949 . The $\triangle k$ for LAW-238 vs VITAMIN-E is thus $\mathbf{0 . 0 0 5 6}$, which is a rather large difference for an infinite medium calculation. Additional study to understand this difference is suggested since the HISS(HUG) calculated $k_{\infty}$ values are in excellent agreement.

\subsection{Fiavion Rate Plots}

A valuable aid to the understanding and analysis of the fast- and thermal-reactor benchmarks considered in this study is to obtain a plot of the fission rate in the fuel zone. Actually, we have plotted fissions/unit lethargy. This method has an advantage of removing the group width $(\Delta U)$ so that each group is on the same basis. The fission rate/unit lethargy plots give an indication of which energy range is important for a given benchmark, but, of course, slowing down is almost always an important consideration so that, for example, scattering in water could be important at higher energies where fissions/unit lethargy may be small. Plots for six fast and three thermal benchmarks are shown in Figs. 7 through 18. For the $\mathrm{il1}$ critical solution benchmark, only $2.1 \%$ of the fissions are above $0.625 \mathrm{eV}$. For the $\mathrm{L} 7$ benchmark, the 


\section{SCALE-238 Ni Total Cross-Section (UH3UR Benchmark)}

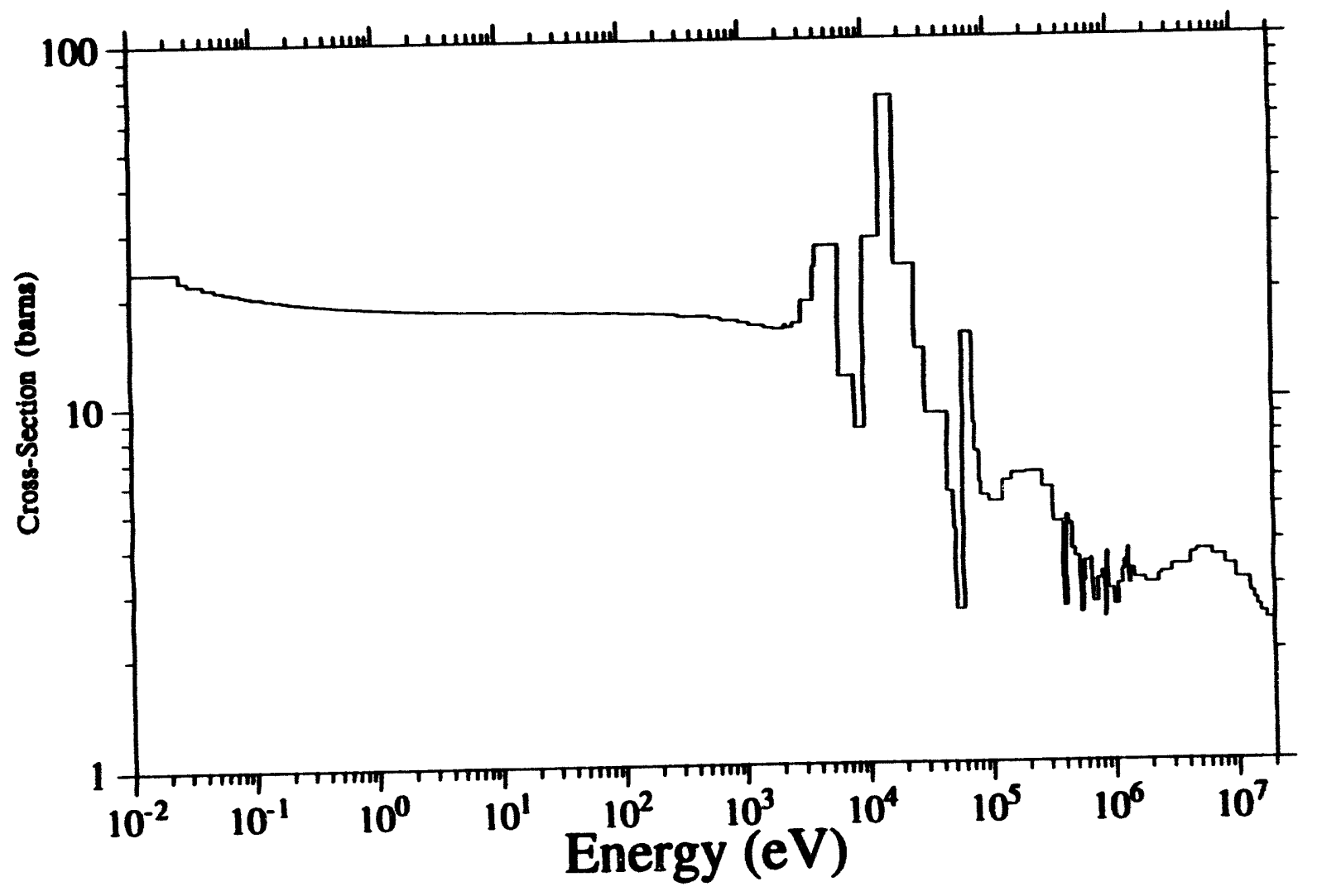

Fig. 5. UH3-NU/SCALE-238 Ni total cross section 


\section{VITAMIN-E Ni Total Cross-Section}

\section{(UH3UR Benchmark)}

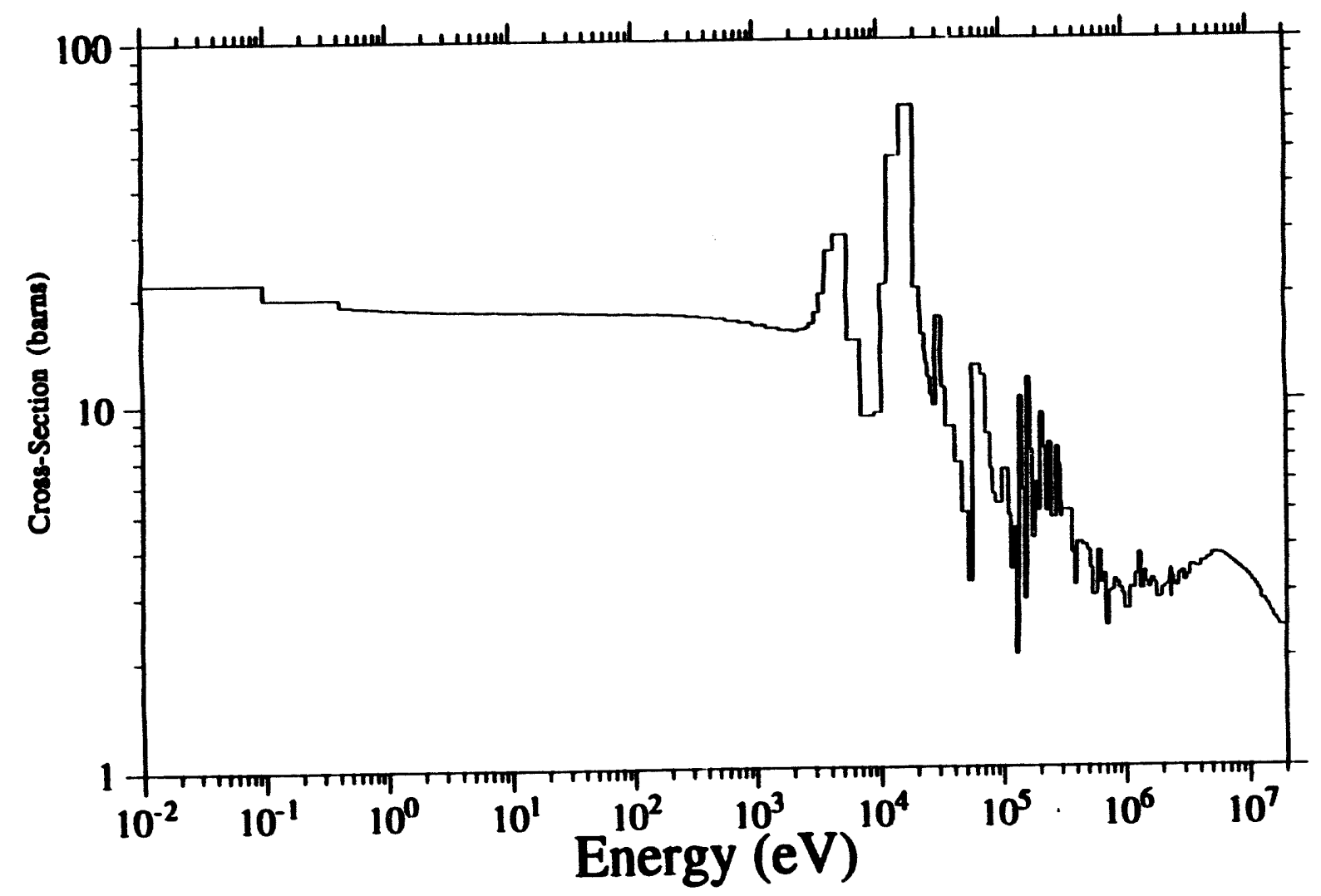

Fig. 6. UH3-NIVITAMIN-E Ni total cross section 


\section{L11CSB BENCHMARK SCALE-238 Library}

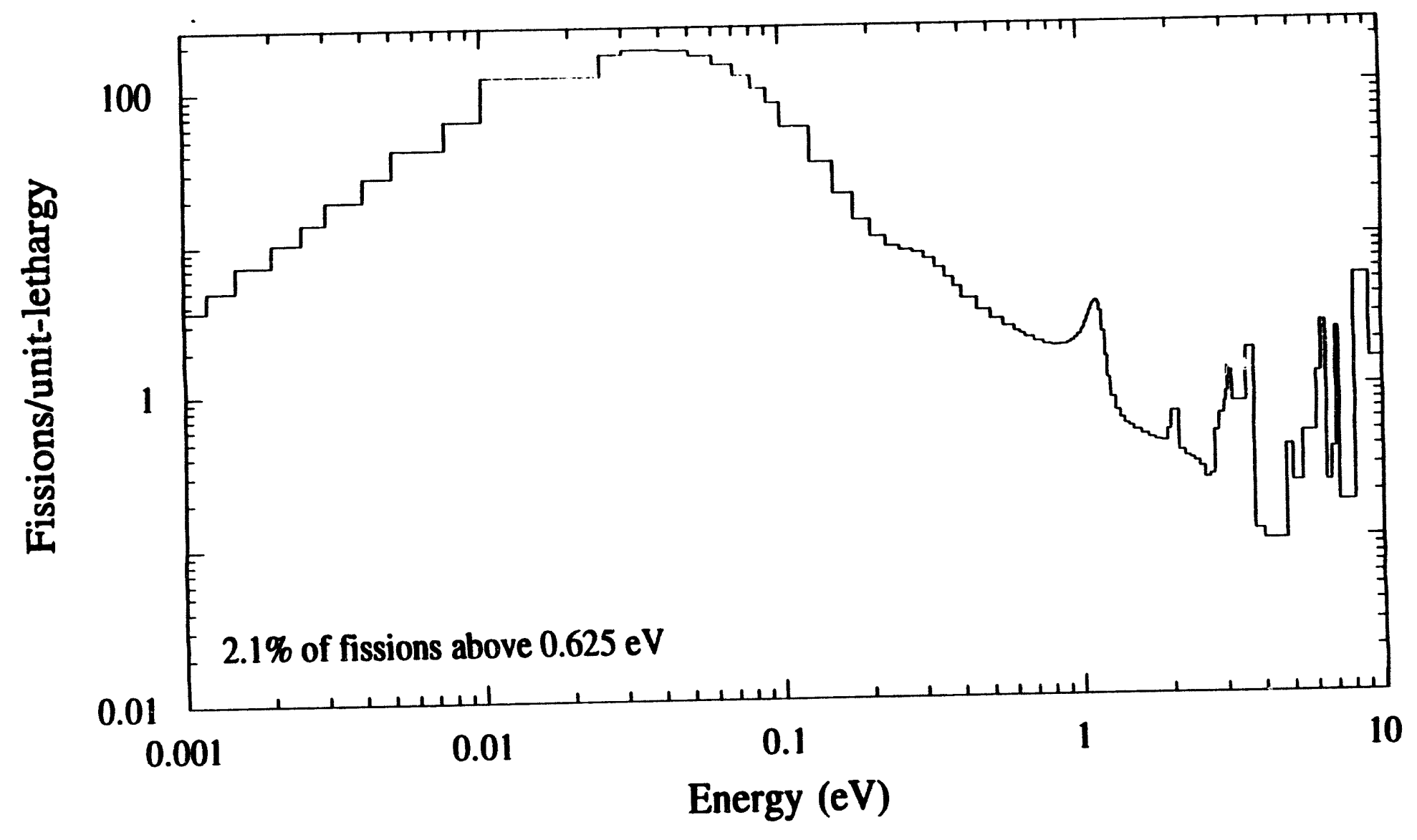

Fig. 7. L11CSB fission rate for 0.001 to $10 \mathrm{eV}$. 


\section{L7CSB BENCHMARK \\ SCALE-238 Library}

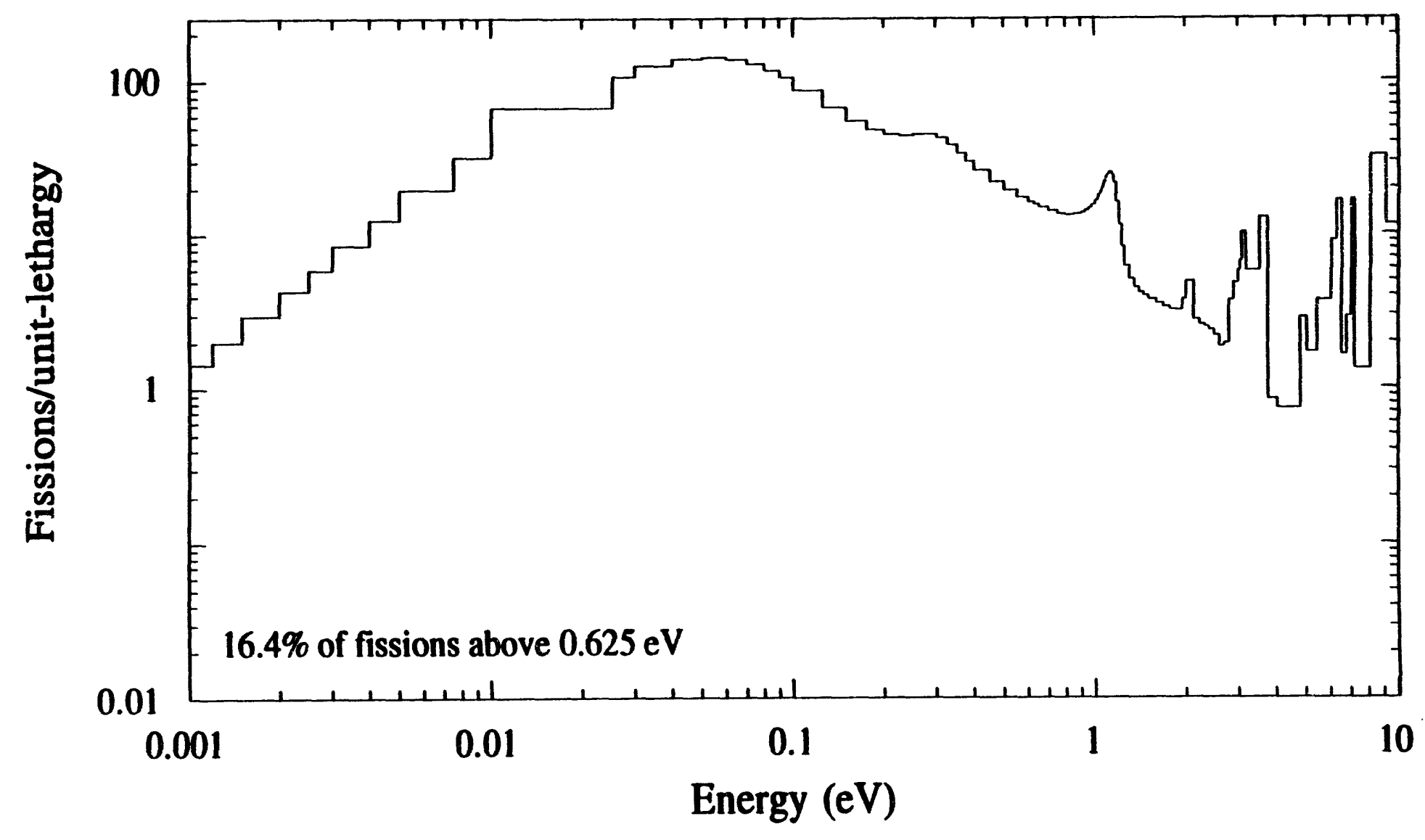

Fig. 8. L7CSB fission rate for 0.001 to $10 \mathrm{eV}$. 


\section{HI240R BENCHMARK}

SCALE-238 Library

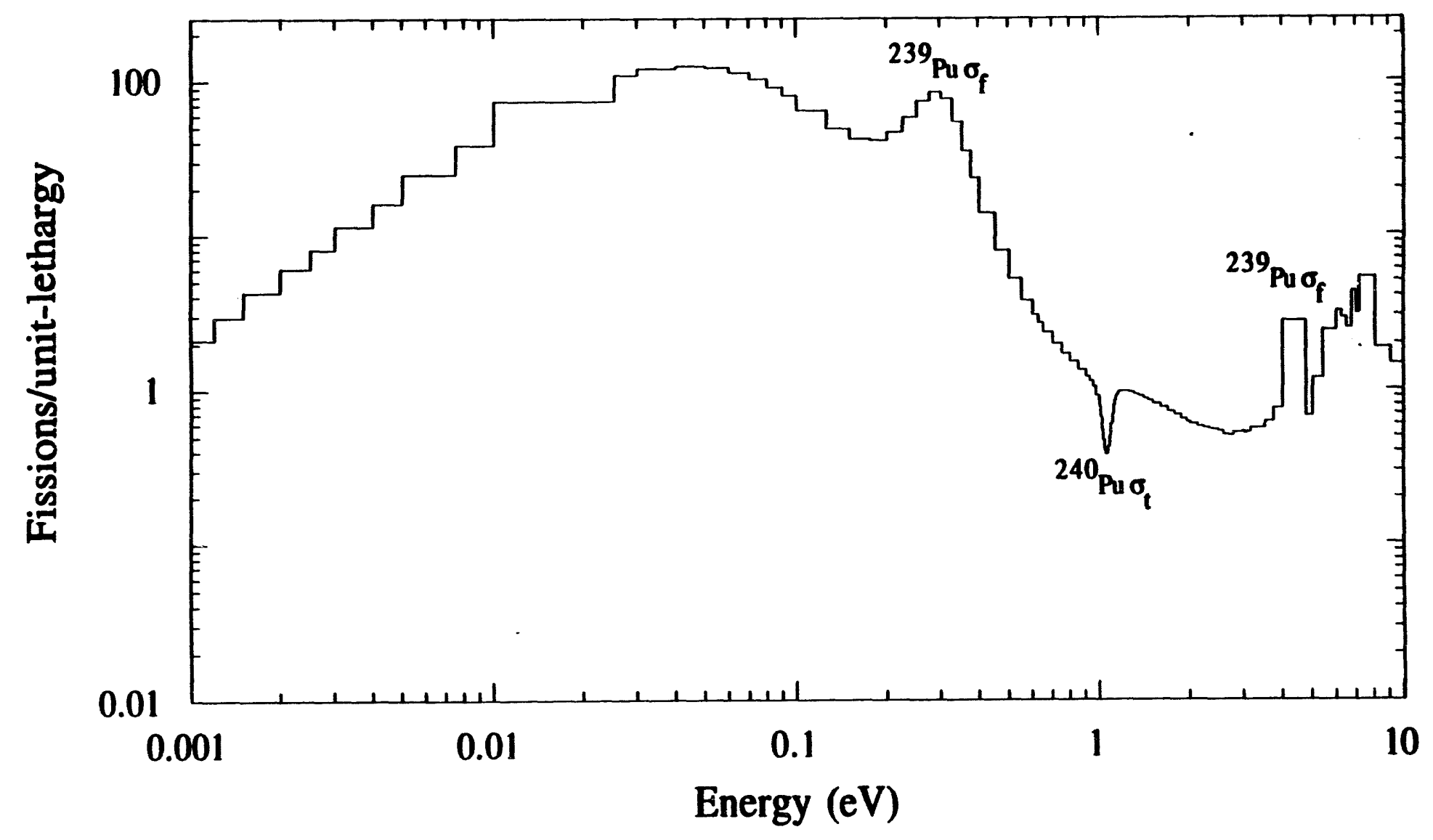

Fig. 9. HI240R fission rate for 0.001 to $10 \mathrm{eV}$. 


\section{H2OX1 BENCHMARK}

SCALE-238 Library

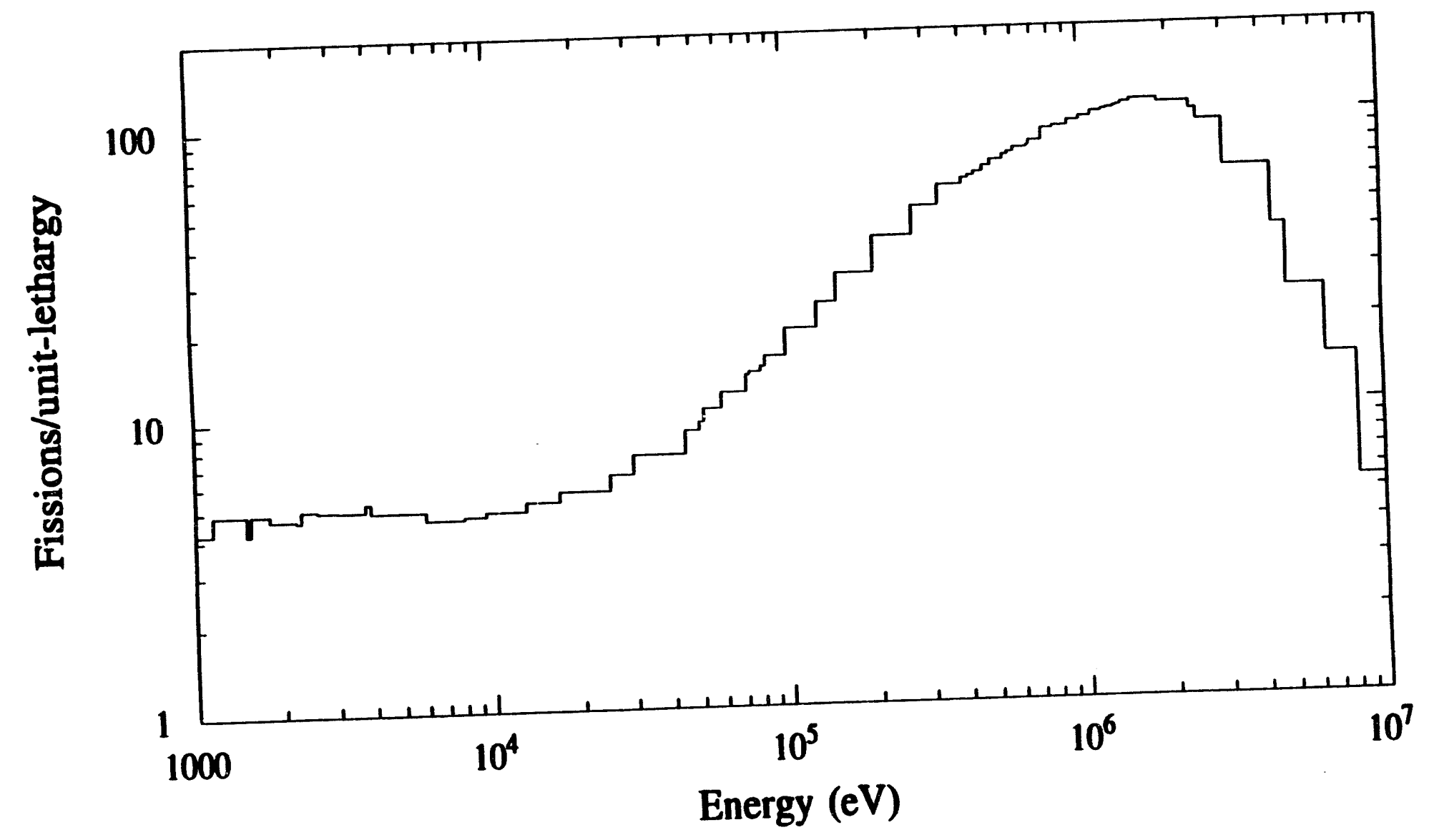

Fig. 10. H2OX1 fission rate for 0.001 to $10 \mathrm{MeV}$. 


\section{H2OX1 BENCHMARK SCALE-238 Library}

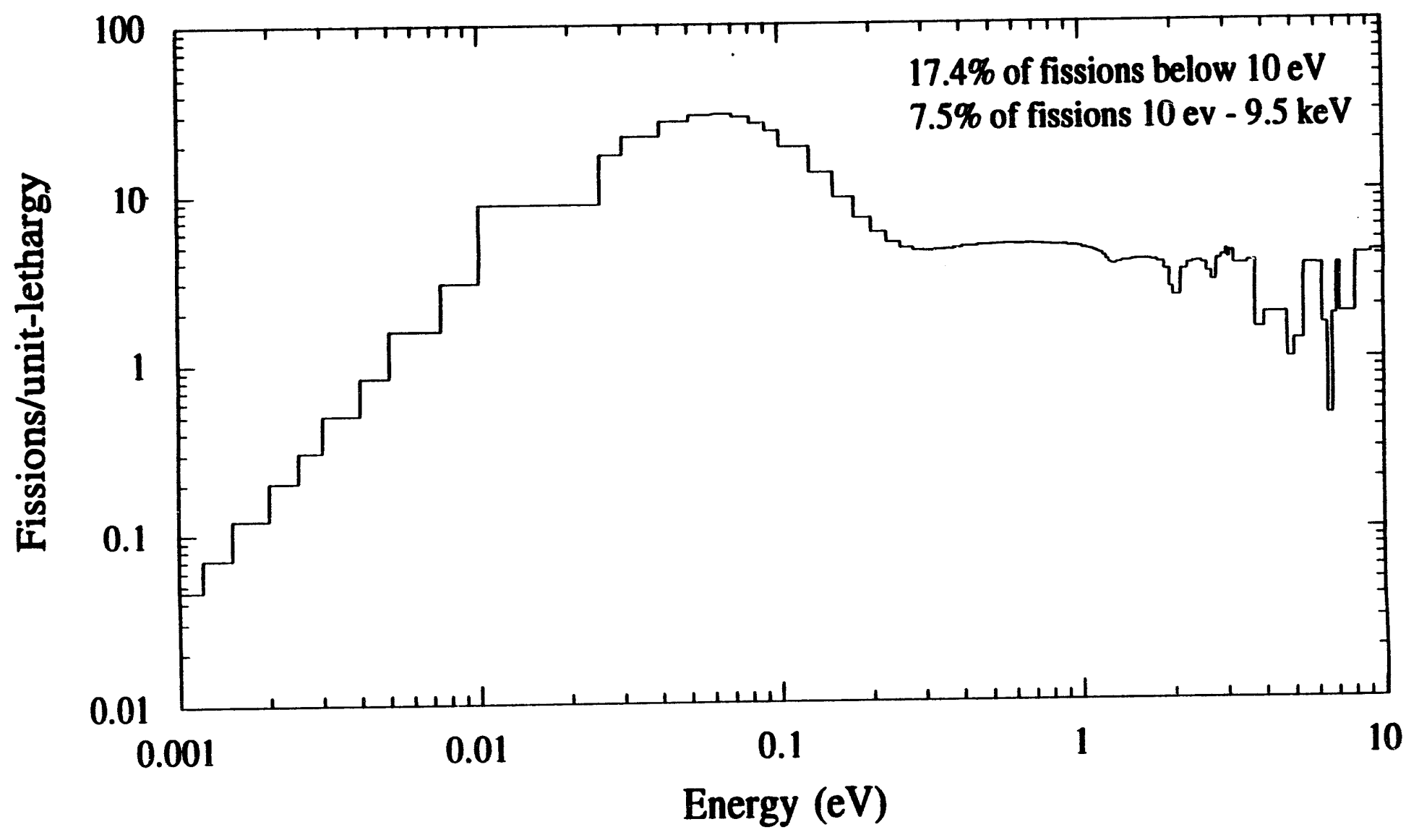

Fig. 11. H2OX1 fission rate for 0.001 to $10 \mathrm{eV}$. 


\section{UH3NI BENCHMARK SCALE-238 Library}

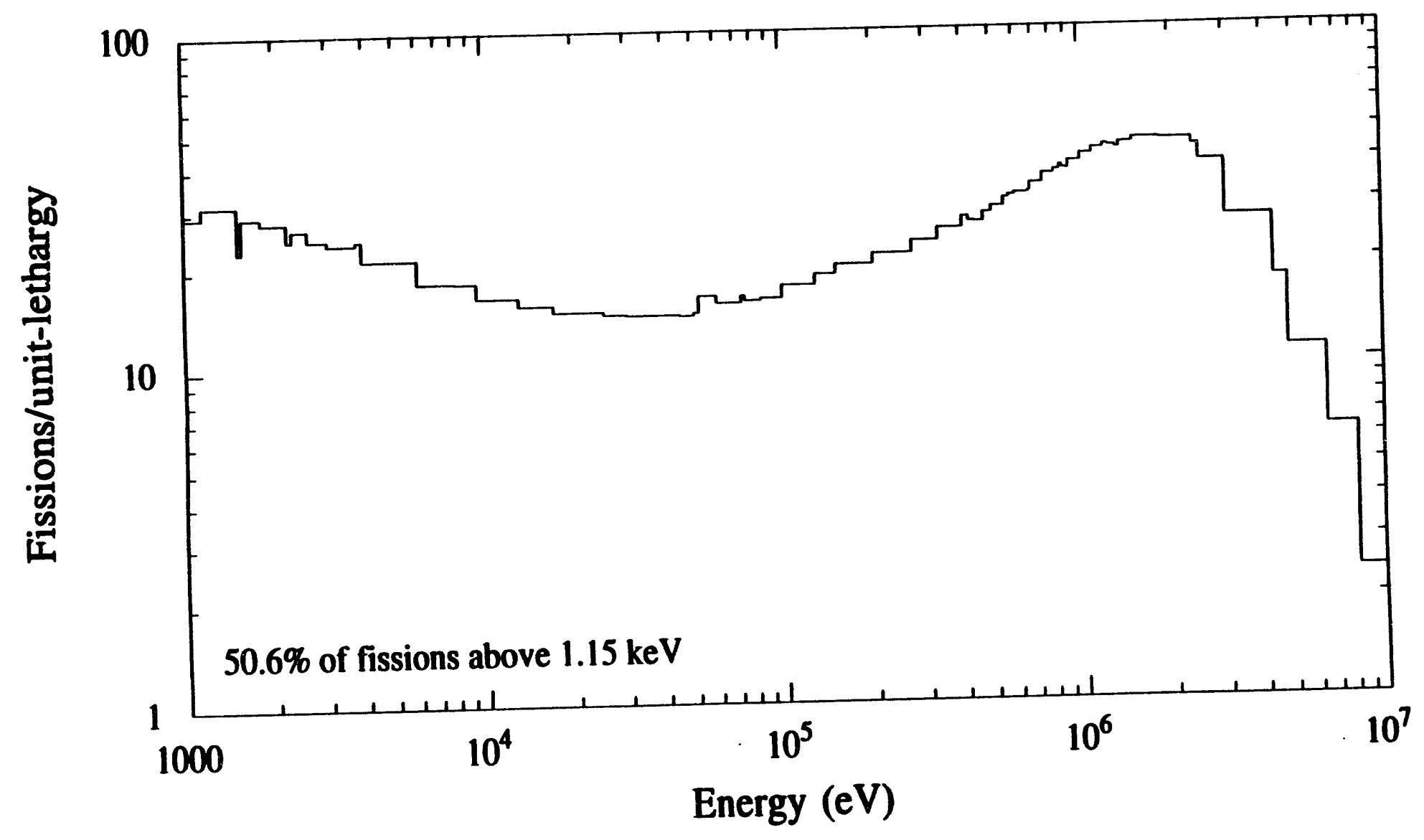

Fig. 12. UH3NI fission rate for 0.001 to $10 \mathrm{MeV}$. 


\section{UH3NI BENCHMARK \\ SCALE-238 Library}

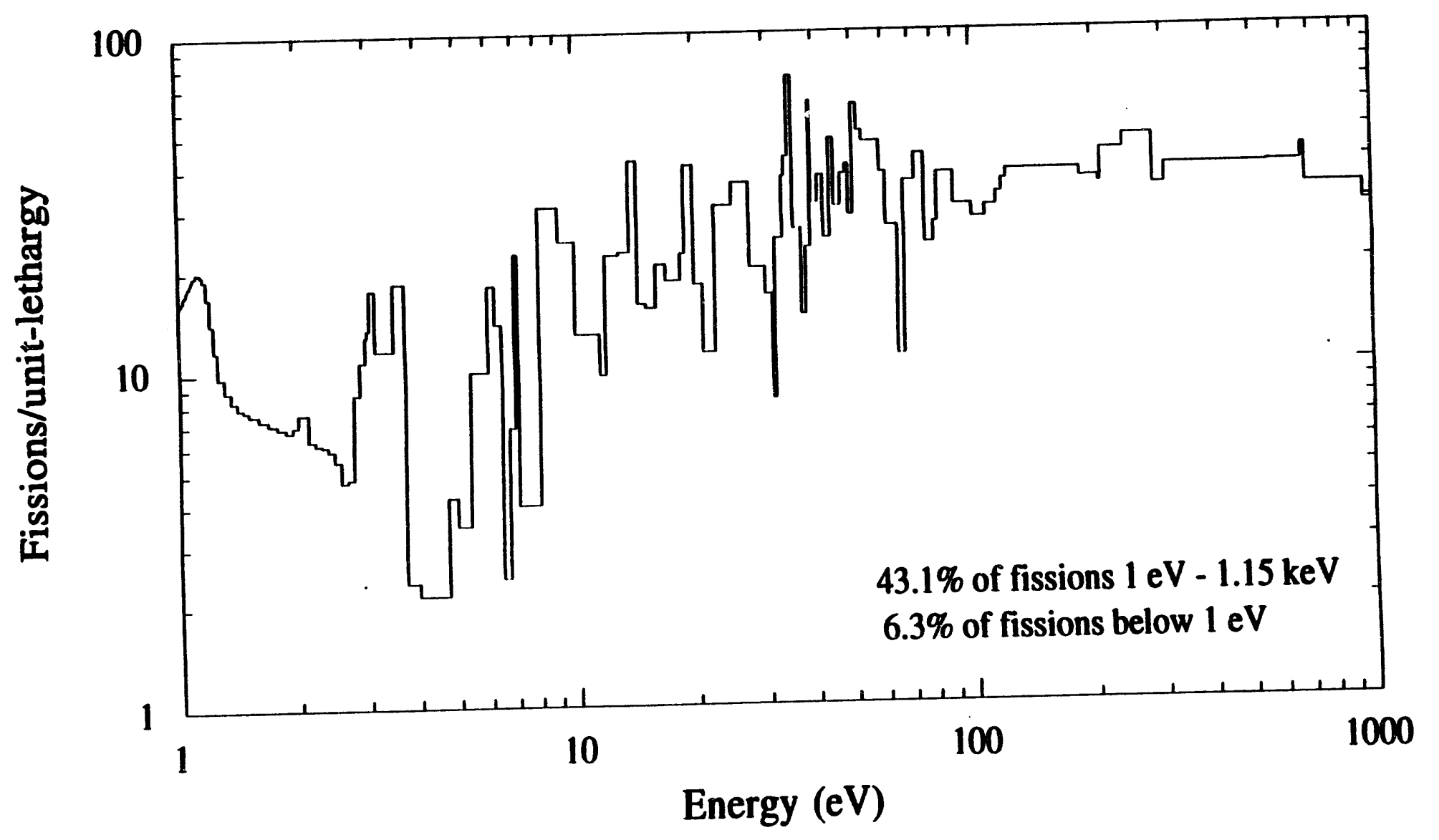

Fig. 13. UH3NI fission rate for 1 to $1000 \mathrm{eV}$. 


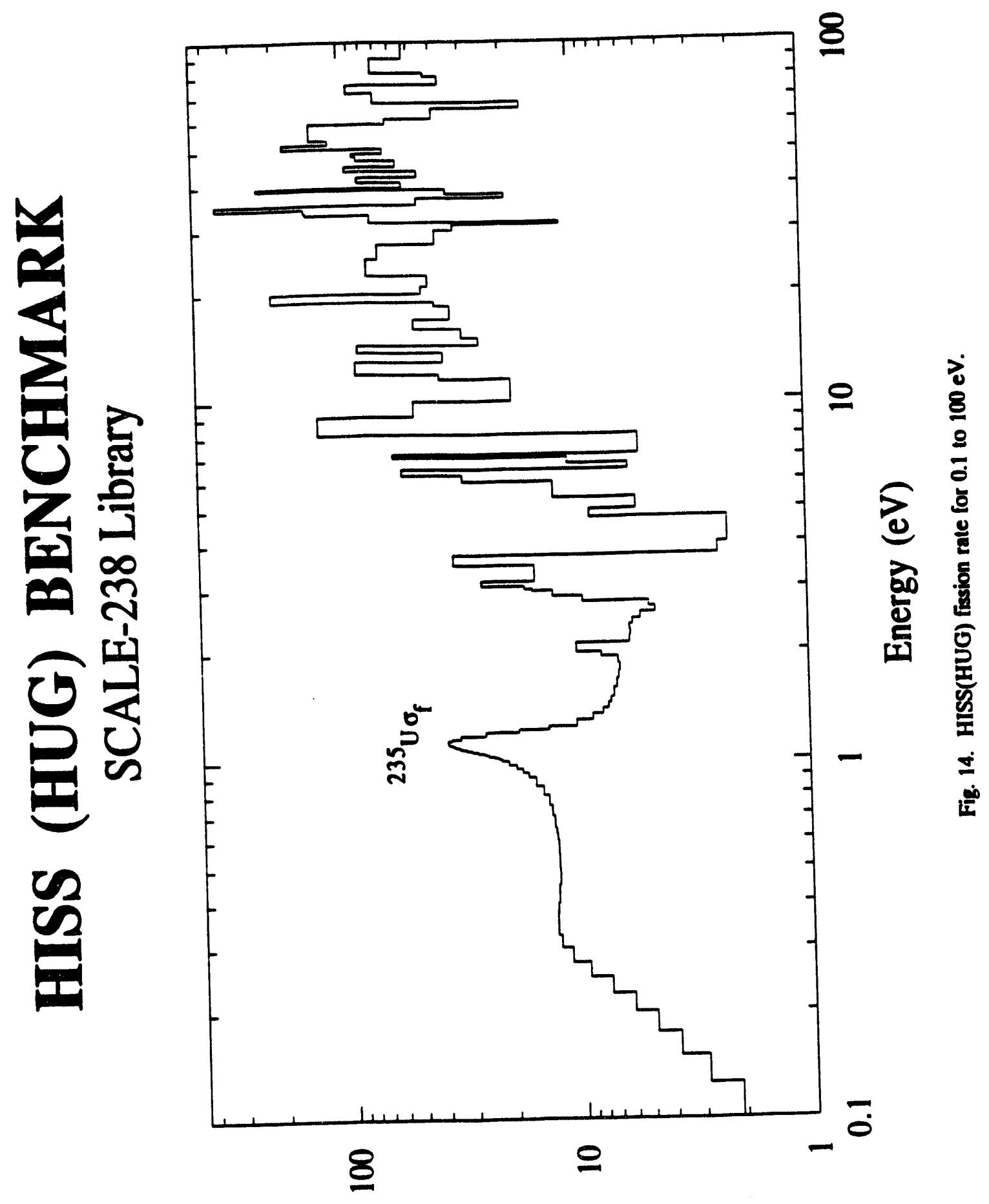

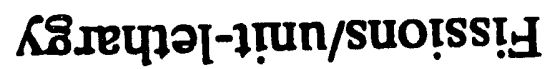




\section{HISS (HUG) BENCHMARK SCALE-238 Library}

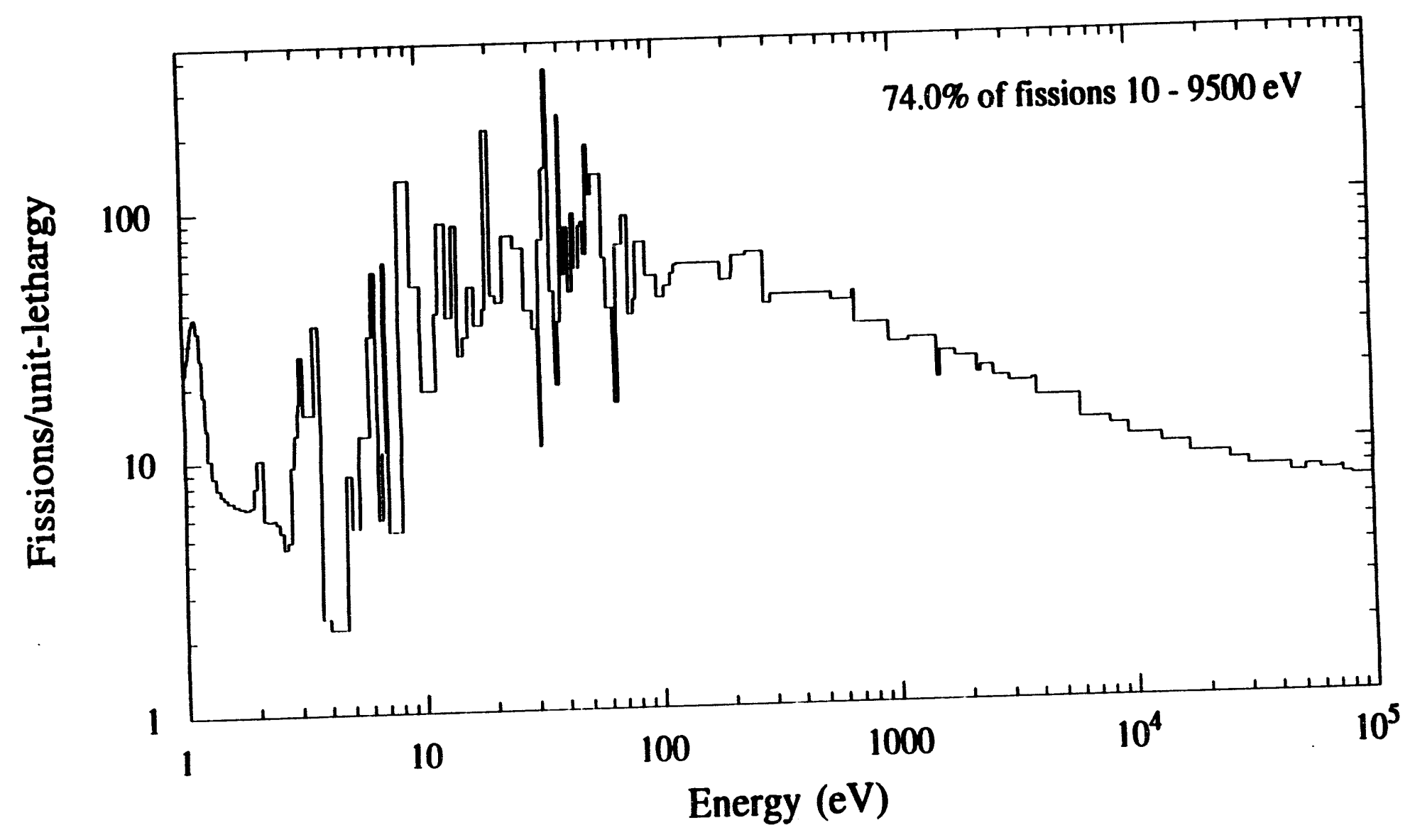

Fig. 15. HISS(HUG) fission rate for 1 to $100,000 \mathrm{eV}$. 


\section{HISS (HPG) BENCHMARK}

SCALE-238 Library

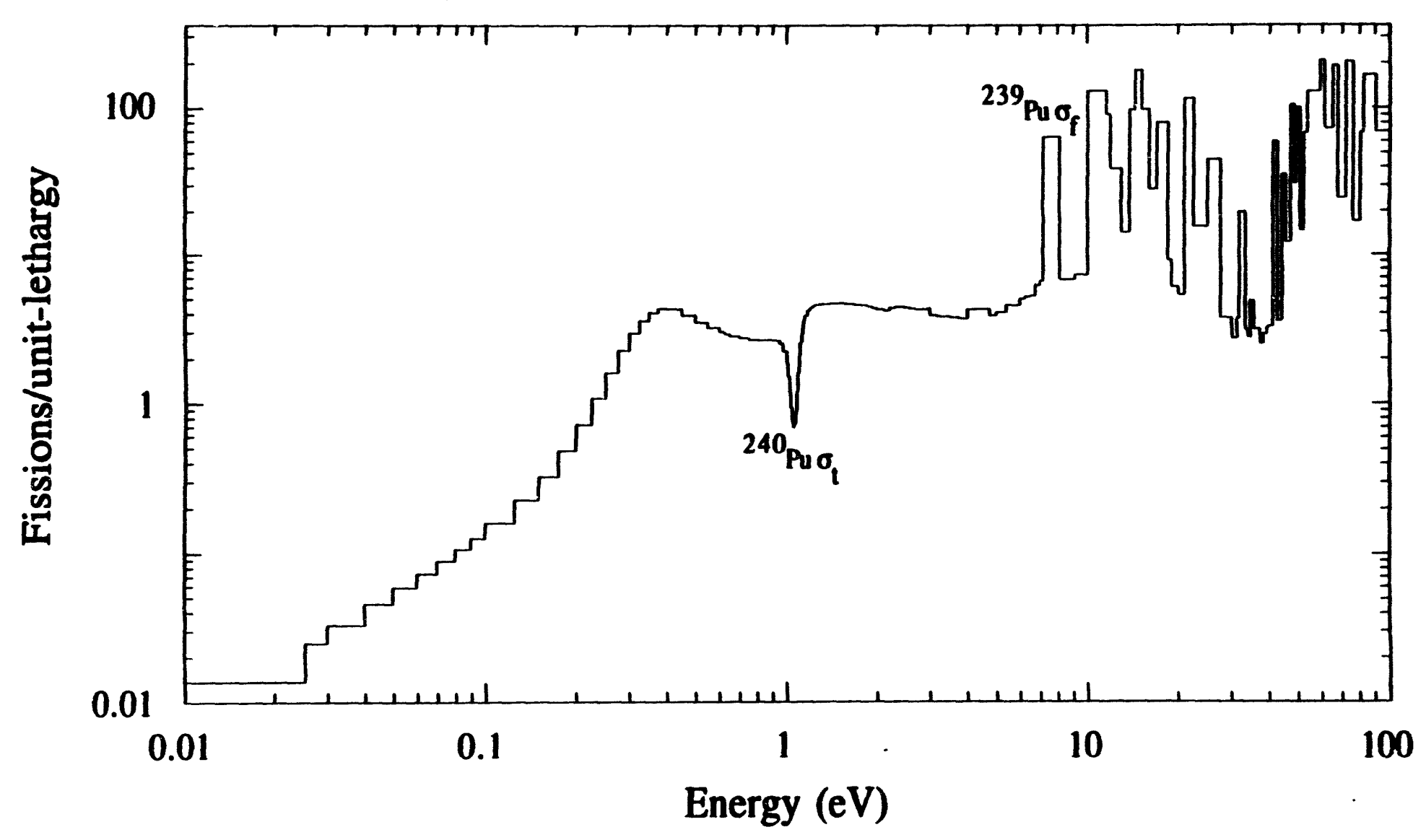

Fig. 16. HISS(HPG) fission rate for 0.1 to $100 \mathrm{eV}$. 


\section{ZPR-3/12 BENCHMARK SCALE-238 Library}

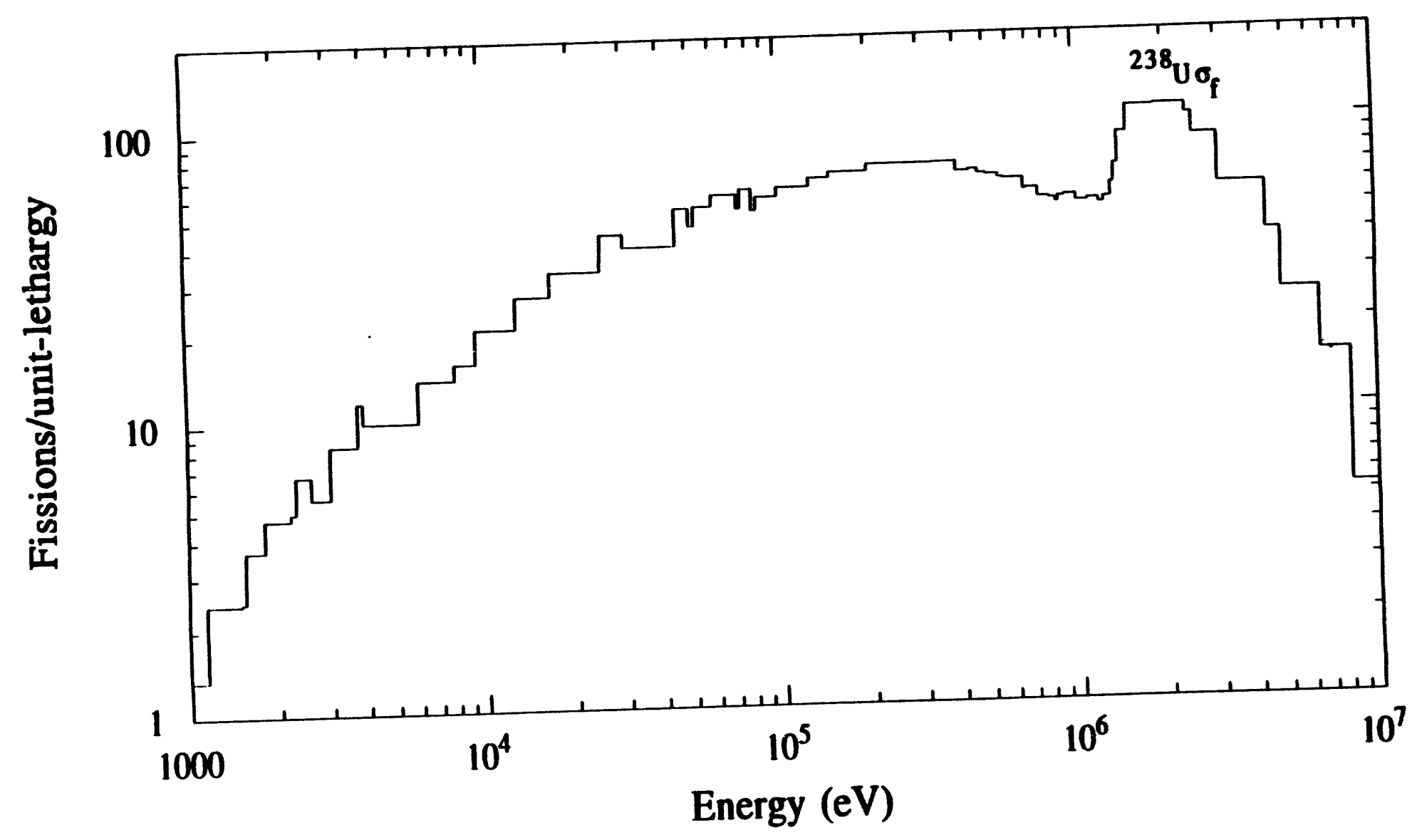

Fig. 17. ZPR-3/12 fission rate for 0.001 to $10 \mathrm{MeV}$. 


\section{ZPR-6/6A BENCHMARK SCALE-238 Library}

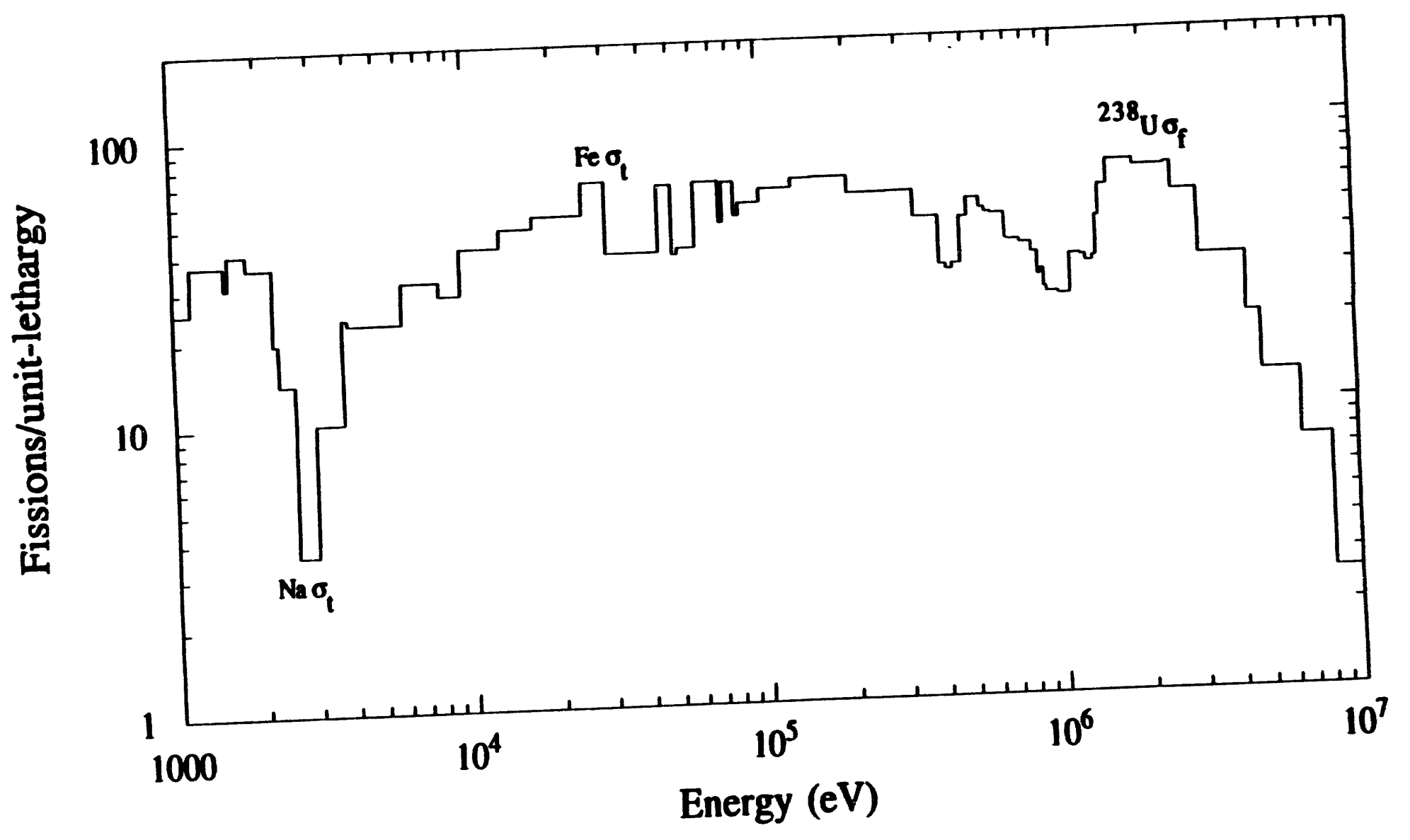

Fig. 18. ZPR-6/6A fission rate for 0.001 to $10 \mathrm{MeV}$. 
benchmark similar to PNL-11 which requires a KENO V.a model. The HI240R benchmark is based on the XSDRNPM spherical approximation to the KENO V.a model. Three interesting features are apparent in the H1240R fission rate plot: ${ }^{20} \mathrm{Pu}$ fission resonance at $0.3 \mathrm{eV} ;{ }^{210} \mathrm{Pu}$ resonance at $1.057 \mathrm{eV}$; and the ${ }^{20} \mathrm{Pu}$ fission rewonance at $7.82 \mathrm{eV}$.

The H2OX1 benchmark is $94 \%$ enriched $\mathrm{U}$-metal with $\mathrm{H}_{2} \mathrm{O}$ reflector. We find that 75.1\% of the fissions are above $9.5 \mathrm{keV}$, and $24.9 \%$ are below. The UH3NI benchmark has uranium hydride fuel with a Ni reflector. We find that $50.6 \%$ of the fissions are above $1.15 \mathrm{keV}$, but $43.1 \%$ of the fissions are in the energy range $1 \mathrm{eV}$ to $1.15 \mathrm{keV}$. Thus, the ${ }^{233} \mathrm{U}$ fission cross section is relatively important in this energy range (i.e., the ${ }^{235} \mathrm{U}$ resolved and unresolved resonance range). For the HISS (HUG) benchmark (homogeneous uranium graphite with a large amount of boron), the fissions/unit lethargy peak in the energy range 30 to $300 \mathrm{eV} ; 74 \%$ of the fissions are in the range 10 to $9500 \mathrm{eV}$. Fissions/unit lethargy fall off below $1 \mathrm{eV}$ because of the high boron content. For the HISS (HPG) benchmark (i.e., homogeneous plutonium graphite with boron), fissions/unit lethargy peak in the energy range 10 to $100 \mathrm{eV}$. ZPR-3/12 is a 4:1 uranium:graphite system, the blanket consists primarily of depleted uranium. The ZPR6/6A benchmark is a uranium-oxide-fueled fast critical assembly. The reflector is $33.81 \mathrm{~cm}$ of depleted uranium. The ZPR-6/6A fuel is enriched uranium with a ${ }^{238} \mathrm{U}$ to ${ }^{235} \mathrm{U}$ ratio of about 5:1. The fuel also contains $\mathrm{Na}, \mathrm{O}$, and $\mathrm{Fe}$, with small amounts of $\mathrm{Ni}, \mathrm{Cr}$, and $\mathrm{Mn}$. A number of features are evident in the fission rate plot (e.g., the sodium resonance at $2.85 \mathrm{keV}$, the iron window at $24 \mathrm{keV}$, and the peak in the fission rate at $2 \mathrm{MeV}$ which is due to ${ }^{238} \mathrm{U}$ fission).

\subsubsection{Calculations with ENDF/B-VI Osygen}

Calculations for ten of the thermal benchmarks using the LAW-238 Library with ENDF/B-VI cross sections for ${ }^{16} \mathrm{O}$ were also done as part of this study. The results are compared with the previous ENDF/B-V calculated values in Table 10. The results using the ENDF/B-VI oxygen are from 0.13 to $0.45 \%$ lower relative to the corresponding ENDF/B-V values. A plot of $k_{\text {off }}$ vo leakage for the L-series benchmarks is shown in Fig. 19; the corresponding ENDF/B-V results are shown in Fig. 4. On the basis of results shown in Fig. 19, the trend for $k_{\text {eff }}$ to increase with leakage is almost eliminated using the ENDF/B-VI oxygen. We note that in Table 10 , $k_{\text {off }}$ for L-9 is $0.45 \%$ higher than for ORNL-1. The reason for this bias is not understood. In any case, it does not seem correct to include the ORNL spheres with the L-series cases in order to show a trend with leakage since each set, by itself, shows almost no trend with leakage. Also, on the basis of the results from this study, the use of ENDF/B-VI cross sections for ${ }^{16} \mathrm{O}$ in the LAW-238 Library is highly recommended.

\subsubsection{Conclusions}

A number of conclusions have been reached concerning the results using the LAW-238 Library in this study. These conclusions are summarized in Table 11. The most important of these is for the UH3-NI benchmark. The LAW-238 group structure is not adequate for the NI reflector of the UH3-NI benchmark. Note the JEZEBEL benchmark (see Table 4); also ZPR-6/6A (Table 4) capture, to agree with the HISS(HUG) experimental value of ${ }^{235} \mathrm{U}$ alpha 
Table 10. Thermal benchmarks with ENDF/B-VI ${ }^{16} \mathrm{O}$

\begin{tabular}{lccc}
\hline & \multicolumn{1}{c}{ All } & \multicolumn{1}{c}{ ENDF/B-VI } & \\
Benchmark & ENDF/B-V & 160 & \% Diff. \\
\hline L-7 & 1.0081 & 1.0036 & -0.45 \\
L-8 & 1.0088 & 1.0065 & -0.23 \\
L-9 & 1.0052 & 1.0032 & -0.20 \\
L-10 & 1.0090 & 1.0046 & -0.44 \\
L-11 & 1.0036 & 1.0013 & -0.23 \\
ORNL-1 & 1.0007 & 0.9987 & -0.20 \\
ORNL-10 & 0.9993 & 0.9980 & -0.13 \\
TRX-1 & & & \\
PNL-3 & 0.9920 & 0.9907 & -0.13 \\
PNL-6B & & & \\
\hline
\end{tabular}




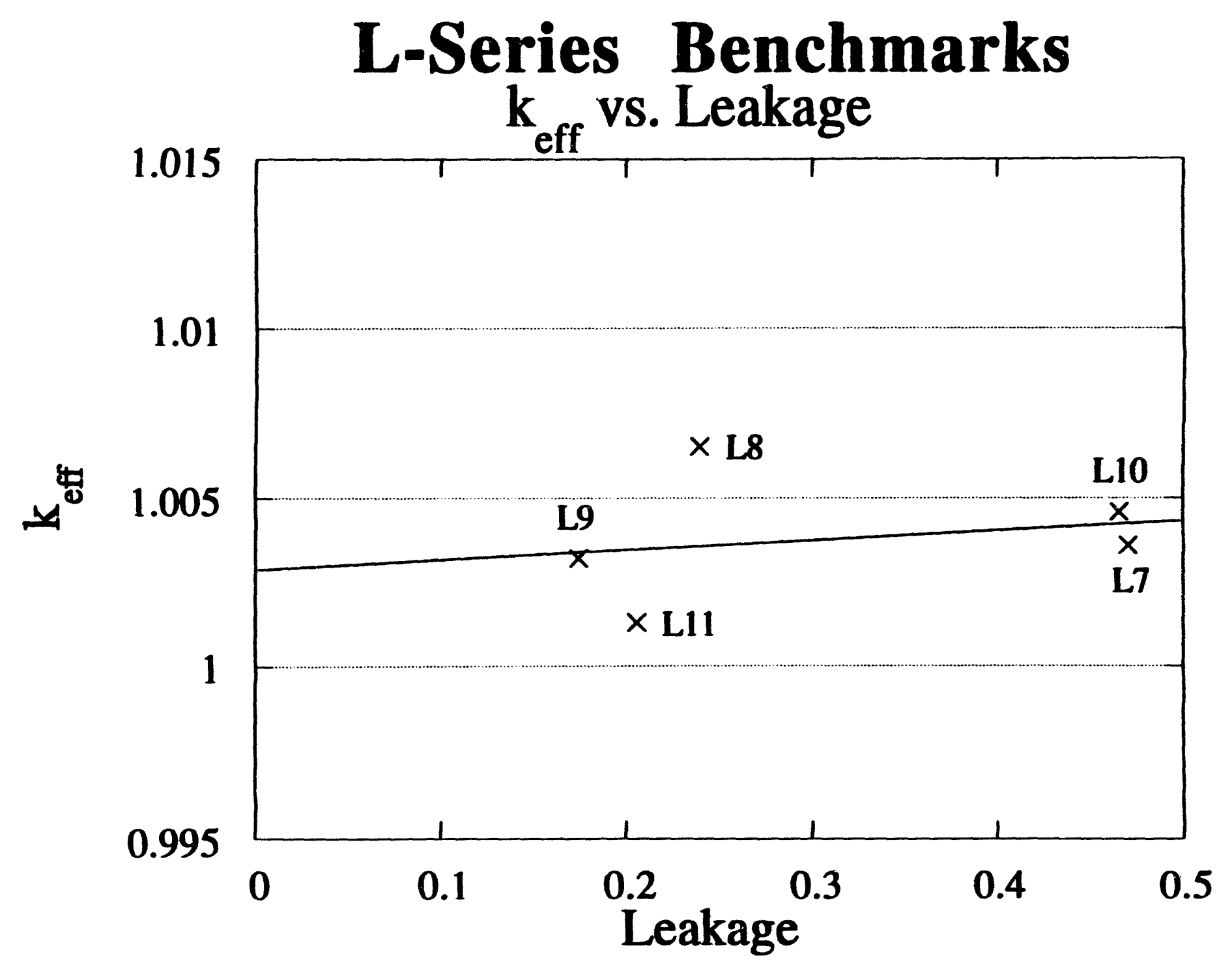

Fig. 19. ket vs leakage with ENDF/B-VI 10 . 
Table 11. Benchmarks for which further study is recommended

\begin{tabular}{|c|c|}
\hline Benchmark & Comment \\
\hline JEZEBEL & LAW-238 value low relative to other ENDF/B-V calculations \\
\hline ZPR-6/6A & LAW-238 value is slightly high relative to other calculations \\
\hline TRX-1 & $\begin{array}{l}\text { LAW-238 } k_{\text {ext }} 7 \text { is } 0.80 \% \text { low relative to the experimental value. See } \\
\text { discussion in the section on uranium lattice benchmark testing for details }\end{array}$ \\
\hline UH3-NI & $\begin{array}{l}\text { LAW-238 group structure is not adequate for the NI reflector. Also } \\
\text { calculate benchmark with ENDF/B-VI cross sections }\end{array}$ \\
\hline HISS(HUG) & $\begin{array}{l}\text { ENDF/B-V }{ }^{235} \text { U fission cross section should be lower? ENDF/B-VI not as } \\
\text { gcod }\end{array}$ \\
\hline HISS(HPG) & LAW-238 value is 0.0056 higher than VITAMIN-E \\
\hline
\end{tabular}

(capture/fission ratio), would significantly improve the agreement between the HISS(HUG) calculated and experimental parameters.

Data testing has been done for 33 benchmarks, including 28 CSEWG benchmarks. Results obtained for these benchmarks are very close to those obtained by other data testers using different ENDF/B-V-based cross-section libraries. There is considerable improvement in the trend of $k_{\text {eff }}$ vs leakage obtained with the use of the ENDF/B-VI oxygen evaluation. The use of the ENDF/B-VI cross sections for ${ }^{16} \mathrm{O}$ in the LAW-238 Library is highly recommended. The LAW-238 Library appears to be acceptable for general use in criticality and reactor physics applications. The library has had minimal testing for shielding applications and should be evaluated by the user for applicability. 


\section{REFERENCES}

1. N. M. Greene et al., AMPX-77: A Modular System for Generating Coupled Multigroup Neutron Gamma Cross-Section Libraries from ENDF/B-IV and/or ENDF-B-V, ORNL/CSD/TM-283, Martin Marietta Energy Systems, Inc., Oak Ridge Natl. Lab., October 1992.

2. R. Kinsey, ed., ENDF/B Summary Documentation, BNL-NCS-17541 (ENDF-201), 3rd ed., Brookhaven National Laboratory, 1979.

3. P. F. Rose and C. L. Dunford, eds., Data Formats and Procedures for the Evaluated Nuclear Data Füle, ENDF, ENDF-102, May 1988.

4. W. E. Ford III et al., CSRL-V: Processed ENDF/B-V 227-Neutron-Group and Pointwise Cross-Section Libraries for Criticality Safety, Reactor and Shielding Studies, NUREG/CR2306 (ORNL/CSD/TM-160), U.S. Nuclear Regulatory Commission, June 1982.

5. SCALE: A Modular Code System for Performing Standardized Computer Analyses for Licensing Evaluation, NUREG/CR-0200, Revision 4 (ORNL/NUREG/CSD-2/R4), Vols. I, II, and III (draft February 1990). Available from Radiation Shielding Information Certer at Oak Ridge National Laboratory as CCC-545.

6. W. A. Rhoades and R. L. Childs, An Updated Version of the DOT4 One-and TwoDimensional Neutron/Photon Transport Code, ORNL-5857, Union Carbide Corp., Nucl. Div., Oak Ridge Natl. Lab., July 1982.

7. W. W. Engle, Jr., A User's Manual for ANISN, K-1693, Union Carbide Corp., Nucl. Div., Oak Ridge Gaseous Diffusion Plant, March 1967.

8. D. R. Vondy, T. B. Fowler, and G. W. Cunningham, VENTURE: A Code Block for Solving Multigroup Neutronics Problems Applying the Finite-Difference Diffusion-Theory Approximation to Neutron Transport, Version II, ORNL-5062/R1, Union Carbide Corp., Nucl. Div., Oak Ridge Natl. Lab., November 1977.

9. E. A. Straker et al., The MORSE Code-A Multigroup Neutron and Gamma-Ray Monte Carlo Transport Code, ORNL/TM-4585, Union Carbide Corp., Nucl. Div., Oak Ridge Natl. Lab., 1970.

10. G. E. Hansen and W. H. Roach, Six and Sixteen Group Cross Sections for Fast and Intermediate Critical Assemblies, LAMS-2543, Los Alamos National Laboratory, 1961.

11. G. D. Joanou and J. S. Dudek, GAM-II: $A B_{3}$ Code for the Calculation of Fast-Neutron Spectra and Associated Multigroup Constants, GA-4265, General Atomics, 1963.

12. H. C. Honeck, THERMOS: A Thermalization Transport Theory Code for Reactor Lattice Calculations, BNL-5826, Brookhaven National Laboratory, September 1961. 
13. W. E. Ford III, R. M. Westfall, and C. C. Webster, "A 218-Neutron-Group Master CrossSection Library for Criticality Safety Studies," Trans. Am. Nucl. Soc. 22, 290 (1975).

14. C. D. West, "Overview of the ANS Project," Trans. Am. Nucl. Soc. 57, 288 (1988).

15. J.-P. Renier, personal communication to N. M. Greene et al., 1992.

16. J. V. Koppel, J. R. Triplell, and Y. D. Naliboff, GASKET: A Unified Code for Thermal Neutron Scattering, GA-7417, General Atomics, September 1966.

17. L. W. Nordheim, "The Theory of Resonance Absorption," Proceedings of Symposia in Applied Mathematics, American Mathematical Soc., Vol. XI, p. 58, Garrett Birkhoff and Eugene P. Wigner, Eds. (1961).

18. I. I. Bondarenko, ed., Group Constants for Nuclear Reactor Calculations, Consultants Bureau, New York (1964).

19. R. E. McFarlane, D. W. Muir, and R. M. Boicourt, The NJOY Nuclear Data Processing System, Volume I: User's Manual, LA-9303-M, Vol. I (ENDF-324), May 1982.

20. Benchmark Data Testing of ENDF/B-V, BNL-NCS-31531 (ENDF-311), Brookhaven National Laboratory, August 1982.

21. Cross Section Evaluation Working Group Benchmark Specifications, BNL-19302 (ENDF202), Brookhaven National Laboratory, November 1974.

22. C. R. Weisbin et al., VTAMIN-E: An ENDF/B-V Multigroup Cross-Section Library for LMFBR Core and Shield, LWR Shield, Dosimetry, and Fusion Blanket Technology, ORNL-5505, Union Carbide Corp., Nucl. Div., Oak Ridge Natl. Lab., February 1979.

23. R. E. MacFarlane, personal communication (May 1992).

24. A. Staub et al., "Analysis of a Set of Critical Homogeneous U-H2O Spheres," Nucl. Sci. Eng. 34, 263 (1968).

25. W. E. Ford III, et al., ANSL-V: ENDF/B-V Based Multigroup Cross-Section Libraries for Advanced Neutron Source (ANS) Reactor Studies, ORNL-6618, Martin Marietta Energy Systems, Inc., Oak Ridge Natl. Lab., September 1990.

26. R. E. MacFarlane, "Eigenvalues Calculated for Homogeneous U-235 Spheres Using All ENDF/B-VI U-235 Data," Attachment 4B-4(1) to Summary of the CSEWG Meeting, May 8-10, 1991, personal communication from C. L. Dunford to Participants.

27. D. S. Craig, Testing of ENDF/B-V Data for Thermal Reactors, AECL-7690 (Rev. 1), Atomic Energy of Canada Ltd., June 1984. 
28. The JOSHUA System, E. I. duPont de Nemours and Co., Savannah River Laboratory, Aiken, SC, USAEC Report DPSTM-500, 1970-1975.

29. OZMA - A Code to Calculate Resonance Reaction Rates in Reactor Lattices Using Resonance Profile Tabulations, EPRI-NP-926, prepared by Technion-Israel Institute of Technology, principal investigators J. Barhen and W. Rothenstein, prepared for Electric Power Research Institute, 1981.

30. R. Q. Wright et al., "Impact of Uncertainties in U-238 Resonance Parameters on Performance Parameters of Thermal Lattices," Trans. Am. Nucl. Soc. 45, 701 (1983).

31. E. Schmidt and P. F. Rose, Testing of the ENDF/B-V Nuclear Data Library in Thermal Benchmark Experiments, EPRI NP-5058, Electric Power Research Institute, February 1987.

32. H. C. Paxton, Los Alamos Critical-Mass Data, LA-3067-MS, Los Alamos Scientific Laboratory, Rev. 1975.

33. G. A. Linenberger et al., "Enriched-Uranium Hydride Critical Assemblies," Nucl. Sci. Eng. 7, 44-57 (1960).

34. W. N. Fox et al., "Reactor Physics Measurements on U-235 and Pu-239 Fuels in an Intermediate Spectrum Assembly," Jour. British Nucl. Energy Soc. 9 (1970). 


\section{APPENDDX A \\ NEW RESONANCE DATA}

ENDF/B-V cross-section evaluations include resolved resonance parameters for higher-order resonances than have historically been included in AMPX and SCALE cross-section libraries. Although Level 0 (s-wave) resonances have always been available for resolved-resonance isotopes, the later ENDF releases have included Level 1 (p-wave), and in some cases Level 2 (d-wave), resonance parameters for a limited number of isotopes. The SCALE LAW-238 cross-section library contains higher-order resonance data not available in earlier SCALE cross-section libraries. The isotopes and the corresponding resonance levels available in the LAW-238 library are given in Table 3 of the text. However, for versions of SCALE prior to and including Version 4.2, the analysis sequences were designed to retrieve only Level 0 data during NITAWL resonance processing. Hence, these versions of SCALE are unable to access the higher-order resonances.

Results presented in this report are based on calculations performed with SCALE 4.2 and therefore include only s-wave $(L=0)$ resonances. However, each of these cases has been recomputed using a modified version of SCALE 4.2 in which the analysis sequence modules have been updated to allow Level-1 and -2 data processing for isotopes for which these data are available. (These modifications will be included in future releases of SCALE, along with enhancements to NITAWL to enable more efficient processing of the higher-order resonances.) Table A.1 compares $\boldsymbol{k}_{\text {eff }}$ values computed using Level-0 data only, as reported in the body of this report, relative to results based on Level- $-0,-1$, and -2 resonance data. These results indicate that use of the higher-order resonance data results in an average decrease in the value of $k_{\mathrm{eff}}$ by approximately $0.2 \%$. However, the higher-order resonances can have a much larger effect on the $k_{\text {eff }}$ value for intermediate energy systems where these nuclides are significant contributors. Inclusion of the higher-order resonances can affect a dry iron $/{ }^{235} U$ mixture by several percent. 
54

Table A.1. Comparisons of results using $L=0$ and $L=0,1$, and 2 resonance data

\begin{tabular}{|c|c|c|c|}
\hline $\begin{array}{l}\text { Case } \\
\text { desiznation }\end{array}$ & $(L=0$ onty) & $\left(L=0,1, k_{\text {end }} 2\right.$ data $)$ & $\%$ change \\
\hline JEZEBEL & 0.9944 & 0.9965 & 0.21 \\
\hline JEZEBEL-PU & 0.9986 & 0.9983 & -0.03 \\
\hline JEZEBEL-23 & 0.9959 & 0.9939 & -0.20 \\
\hline GODIVA & 0.9963 & 0.9980 & 0.17 \\
\hline FLATTOP-25 & 1.0046 & 1.0046 & 0.00 \\
\hline BIG TEN & 1.0103 & 1.0103 & 0.00 \\
\hline ZPR-3/11 & 1.0119 & 1.0119 & 0.00 \\
\hline ZPR-3/12 & 1.0068 & 1.0071 & 0.03 \\
\hline ZPR-6/6A & 0.9952 & 0.9981 & 0.29 \\
\hline ZPR-6/7 & 1.0022 & 0.9367 & -0.55 \\
\hline ORNL-1 & 1.0007 & 0.9987 & -0.20 \\
\hline ORNL-2 & 1.0005 & 0.9985 & -0.20 \\
\hline ORNL-3 & 0.9975 & 0.9955 & -0.20 \\
\hline ORNL-4 & 0.9989 & 0.9969 & -0.20 \\
\hline ORNL-10 & 0.9993 & 0.9980 & -0.13 \\
\hline TRX-1 & 0.9920 & 0.9908 & -0.12 \\
\hline TRX-2 & 0.9962 & 0.9946 & -0.16 \\
\hline BAPL-1 & 0.9986 & 0.9921 & -0.65 \\
\hline BAPL-2 & 1.0002 & 0.9938 & -0.64 \\
\hline BAPL-3 & 1.0024 & 0.9963 & -0.61 \\
\hline L7 & 1.0081 & 1.0031 & -0.50 \\
\hline L-8 & 1.0088 & 1.0064 & -0.24 \\
\hline L-9 & 1.0052 & 1.0032 & -0.20 \\
\hline L-10 & 1.0090 & 1.0042 & -0.48 \\
\hline L-11 & 1.0036 & 1.0012 & -0.24 \\
\hline PNL-3 & 0.9982 & 0.9951 & -0.31 \\
\hline PNL-6B & 1.0101 & 1.0057 & -0.44 \\
\hline PNL-11 & $1.0023 \pm 0.0034$ & $1.0000 \pm 0.0034$ & -0.23 \\
\hline H2OX-1 & 1.0047 & 1.0006 & -0.41 \\
\hline UH3-UR & 1.0098 & 1.0112 & 0.14 \\
\hline UH3-NI & 1.0245 & 1.0223 & -0.21 \\
\hline HISS(HUG) & 1.0241 & 1.0241 & 0.00 \\
\hline HISS(HPG) & 1.0005 & 1.0005 & 0.00 \\
\hline Average & 1.0034 & 1.0015 & -0.19 \\
\hline Morimum & 1.0245 & 1.0241 & \\
\hline Minimum & 0.9920 & 0.9908 & \\
\hline
\end{tabular}




\section{INTERNAL DISTRIBUTION}

1. C. W. Alexander

2. J. W. Arwood

3. S. M. Bowman

4. B. L. Broadhead

5. J. A Bucholz

6. R. L. Childs

7. A. G. Croff

8. E. C. Crume

9. R. D. Dabbs

10. M. D. DeHart

11. H. L. Dodds

12. H. R. Dyer

13. M. B. Emmett

14. C. W. Forsberg

15-19. N. M. Greene

20. O. W. Hermann

21. D. Hollenbach

22. C. M. Hopper

23. D. T. Ingersoll

24. W. C. Jordan

25. F. B. Kam

26. N. F. Landers

27. L. C. Leal

28. S. B. Ludwig

29. J. B. Manneschmidt

30. G. E. Michaels

31. B. D. Murphy
32. L. F. Norris

33-37. C. V. Parks

38. L. M. Petrie

39. R. T. Primm

40. J.-P. Renier

41. R. C. Robinson

42. R. B. Rothrock

43. R. W. Roussin

44. J. C. Ryman

45. C. H. Shappert

46. O. L. Smith

47. G. R. Smolen

48. R. V. Stachowiak

49. J. S. Tang

50. R. G. Taylor

51. D. A. Tollefson

52. R. G. Vornehm

53. R. M. Westfall

54. J. E. White

55. G. E. Whitesides

56. B. A. Worley

57-61. R. Q. Wright

62. Central Research Library

63-64. ORNL Y-12 Research Library Document Reference Section

65. Laboratory Records Department

66. Laboratory Records, ORNL (RC)

67. ORNL Patent Office

\section{EXTERNAL DISTRIBUTION}

68. F. Alcorn, Babcock \& Wilcox - NNFD, P.O. Box 785, M/C 46, Lynchburg, VA 24505

69. M. G. Bailey, Office of Nuclear Material Safety \& Safeguards, U.S. Nuclear Regulatory Commission, MS TWFN-8F5, Washington, DC 20555

70. W. M. Blumberg, Houston Lighting \& Power Company, South Texas Project Electric Generating Station, P.O. Box 289, Wadsworth, TX 77483

71. J. K. Boshoven, General Atomics, 3550 General Atomics Court, San Diego, CA 92121-1194

72. M. C. Brady, Sandia National Laboratories, 101 Convention Center Drive, Suite 880, Las Vegas, NV 89109 
73. R. Carison, Lawrence Livermore National Laboratory, P.O. Box 808, Livermore, CA 94550

74. Jor-Shan Choi, Lawrence Livermore National Laboratory, P.O. Box 808, L-634, Livermore, CA 94550

75. P. Felsher, EG\&G Rocky Flats, P.O. Box 464, Bldg. 886, Golden, CO 80402 0464

76. I. Fergus, U.S. Department of Energy, MS EH-11, Washington, DC 20485

77. M. N. Haas, West Valley Nuclear Services Co., Inc., P.O. Box 191, West Falley, NY 14171-0191

78. K. J. Hardin, Office of Nuclear Material Safety \& Safeguards, U.S. Nuclear Regulatory Commission, MS TWFN-8A-33, Washington, DC 20555

79. N. Kent, Westinghouse Electric Corp., P.O. Drawer R, Columbia, SC 29250

80. M. Klasky, Office of Nuclear Material Safety \& Safeguards, U.S. Nuclear Regulatory Commission, MS TWFN-8A-33, Washington, DC 20555

81. M. Mason, Transnuclear, Inc., 2 Skyline Drive, Hawthorne, NY 10532

82. J. Mincey, Westinghouse Savannah River Company, Building 773-22A, Aiken, SC 29808

83. R. Mosteller, Los Alamos National Laboratory, P.O. Box 1663, N-12, MS K551, Los Alamos, NM 87545

84. P. K. Nair, Manager, Engineered Barrier System, Center for Nuclear Waste Regulatory Analyses, Southwest Research Institute, 6220 Culebra Road, San Antonio, TX 78238-5166

85. D. Napolitano, 655 Engineering Drive, Suite 200, Norcross, GA 30092

86. Office of the Deputy Assistant Manager for Energy, Research, and Development, U.S. Department of Energy, Oak Ridge Operations (DOE-ORO), P.O. Box 2001, Oak Ridge, TN 37831

87-88. Office of Scientific and Technical Information, P.O. Box 62, Oak Ridge, TN 37831

89. F. Prohammer, Argonne National Laboratory, 9700 S. Cass Ave., Bldg. 308, Argonne, II. 60439-4825

90. M. Rahimi, 2650 Park Tower Drive, Suite 800, Vienna, VA 22180

91. M. K. Sheaffer, Lawrence Livermore National Laboratory, P.O. Box 808, L-197, Livermore, CA 94550

92. M. L. Smith, 5000 Dominion Blvd., Glen Allen, VA 23060

93. H. Taniuchi, Kobe Steel, Ltd., 3-1, Araicho-Shinhama, 2-Chome, Takasago, Hyogo, 676 Japan

94. J. T. Taylor, WINCO (INEL), P.O. Box 4000, MS 3428, Idaho Falls, ID 83403

95. D. A. Thomas, B\&W Fuel Co., 101 Convention Center Drive, Suite 527, MS 423, Las Vegas, NV 89109

96. J. R. Thornton, Duke Engineering \& Services, Inc., 2300 S. Tryon St., P.O. Box 1004, Charlotte, NC 28201-1004

97. H. Toffer, Westinghouse Hanford Company, P.O. Box 1970, HO-38, Richland, WA 99352

98-99. M. E. Wangler, U.S. Department of Energy, Transportation \& Packaging Safety Div., MS EH-332, Washington, DC 20585

100. F. G. Welfare, General Electric Co., Nuclear Fuel Manufacturing Dept., P.O. Box 780, Wilmington, NC 28402

101. A. Wells, 2846 Peachtree Walk, Duluth, GA 30136

102. B. H. White, Office of Nuclear Material Safety \& Safeguards, U.S. Nuclear Regulatory Commission, MS TWFN-8F5, Washington, DC 20555 
103. M. L. Williams, Nuclear Science Center, Louisiana State University, Baton Rouge, LA 70803

104. R. E. Wileon, Fuel Cycle Safety Branch, Office of Nuclear Material Safety and Safeguands, U.S. Nuclear Regulatory Commission, MS 6H3, Washington, DC 20555

105. C. J. Withee, Nuclear Material Safety \& Safeguards, U.S. Nuclear Regulatory Commission, MS WF1-4EA, Washington, DC 20555 

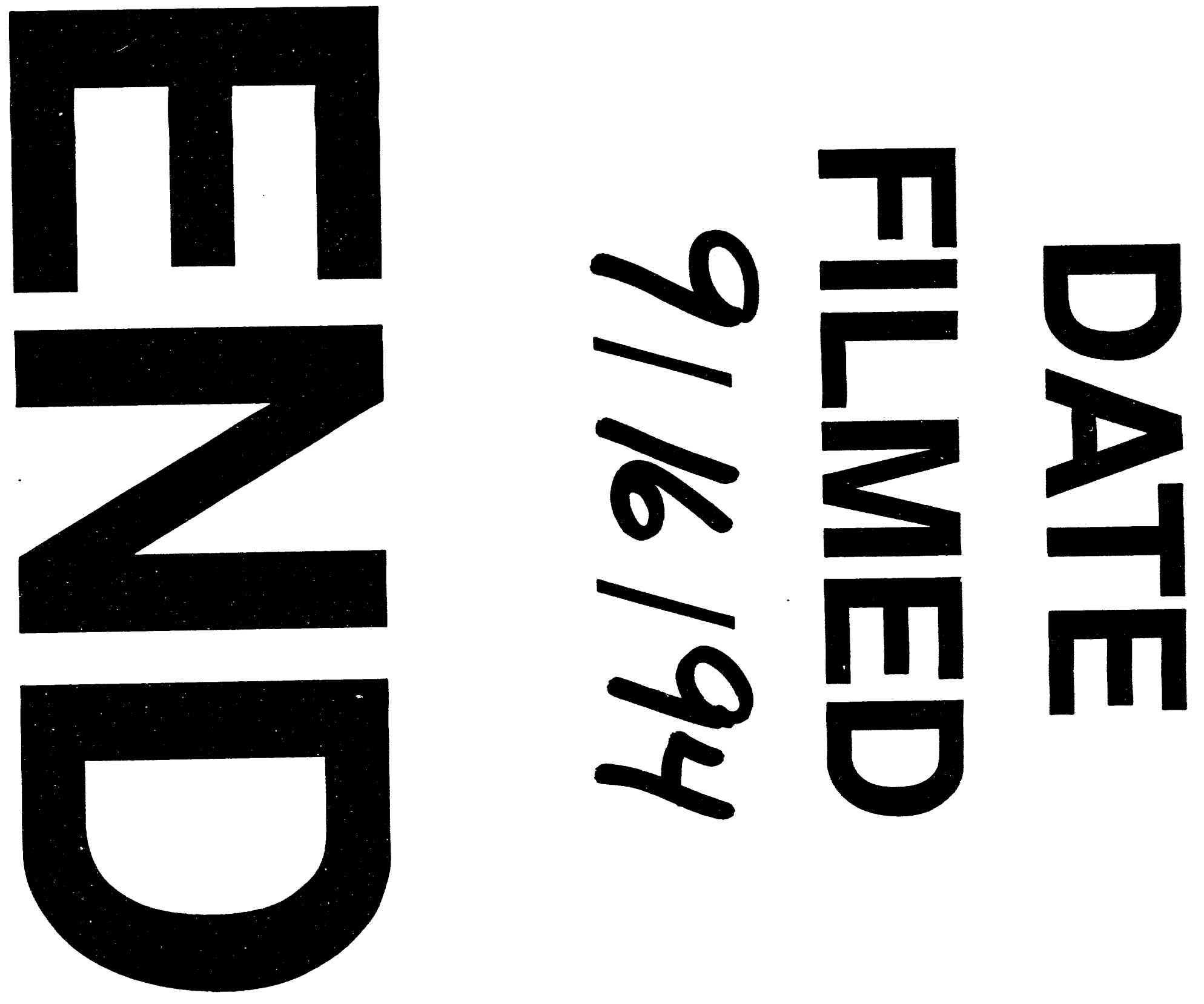
